\title{
Properties and Evaluation of the MOBIT - a novel Linkage-based Test Statistic and Quantification Method for Imprinting
}

\author{
${ }^{1}$ Chair of Genetic Epidemiology, IBE, Faculty of Medicine, LMU Munich, Munich, Germany, E-mail: \\ markus.brugger@helmholtz-muenchen.de \\ ${ }^{2}$ Institute of Cenetic Epidemiology, Helmholtz Zentrum München - Cerman Research Center for Environmental Health, In- \\ golstädter Landstraße 1, DE-85764 Neuherberg, Germany, E-mail: markus.brugger@helmholtz-muenchen.de \\ ${ }^{3}$ Institute for Medical Biometry, Informatics and Epidemiology, University of Bonn, Venusberg-Campus 1, DE-53127 Bonn, Ger- \\ many
}

\begin{abstract}
:
Genomic imprinting is a parent-of-origin effect apparent in an appreciable number of human diseases. We have proposed the new imprinting test statistic MOBIT, which is based on MOD score analysis. We were interested in the properties of the MOBIT concerning its distribution under three hypotheses: (1) $H_{0, a}:$ no linkage, no imprinting; (2) $\mathrm{H}_{0, b}$ : linkage, no imprinting; (3) $\mathrm{H}_{1}$ : linkage and imprinting. More specifically, we assessed the confounding between imprinting and sex-specific recombination frequencies, which presents a major difficulty in linkage-based testing for imprinting, and evaluated the power of the test. To this end, we have performed a linkage simulation study of affected sib-pairs and a three-generation pedigree with two trait models, many twoand multipoint marker scenarios, three genetic map ratios, two sample sizes, and five imprinting degrees. We also investigated the ability of the MOBIT to quantify the degree of imprinting and applied the MOBIT using a real data example on house dust mite allergy. We further proposed and evaluated two approaches to obtain empiric $p$ values for the MOBIT. Our results showed that twopoint analyses assuming a sex-averaged marker map led to an inflated type I error due to confounding, especially for a larger marker-trait locus distance. When the correct sex-specific marker map was assumed, twopoint analyses have a reduced power to detect imprinting, compared to sex-averaged analyses with an appropriate correction for the inflation of the test statistic. However, confounding was not an issue in multipoint analysis unless the map ratio was extreme and marker spacing was sparse. With multipoint analysis, power as well as the ability to quantify the imprinting degree were almost equally high when a sex-averaged or the correct sex-specific map was used in the analysis. We recommend to obtain empiric $p$ values for the MOBIT using genotype simulations based on the best-fitting nonimprinting model of the real dataset analysis. In addition, an implementation of a method based on the permutation of parental sexes is also available. In summary, we propose to perform multipoint analyses using densely spaced markers to efficiently discover new imprinted loci and to reliably quantify the degree of imprinting.
\end{abstract}

Keywords: confounding, genomic imprinting, linkage analysis, MOD scores, sex-specific recombination frequencies

DOI: $10.1515 /$ sagmb-2018-0025

\section{Introduction}

The human genetic map length differs between males and females. This is possibly due to mechanisms closely related to those of genomic imprinting, as regions with sex-specific recombination frequencies often coincide with imprinted ones (Pàldi, Gyapay \& Jami, 1995). Genomic imprinting is an epigenetic phenomenon which is present in all viviparous mammals, some plants and, in a wider sense, some insects like the scale insect Sciara coprophila, in which it was first described in 1938 (Metz, 1938). As genomic imprinting means the dependence of an individual's liability to develop a disease according to the parental origin of the mutated allele(s), it leads to a deviation from the classic Mendelian assumption of equal contribution of parental genomes to the progeny and is therefore called a parent-of-origin effect (Falls et al., 1999). The degree of genomic imprinting can range from complete inactivation to reduced expression of the respective gene and is established in a time- and tissue-specific manner. Genomic imprinting is caused by DNA and histone modifications without 
changing the nucleotide sequence, i.e. it is epigenetic, and is controlled by imprinting centers (ICs) containing differentially-methylated regions (DMRs) (Lewis \& Reik, 2006; Spencer, 2009). Apart from being involved in embryonic development, such that parthenogenetic embryos which arise from genomes of the same parent are hindered to survive to birth, more and more evidence emerges hinting at imprinting being involved in many diseases in the adult. Amongst these are Angelman, Beckwith-Wiedemann, Prader-Willi (Walter \& Paulsen, 2003) and Silver-Russell syndrome (Solter, 2006) as well as complex traits like type I diabetes (Bain et al., 1994), atopy (Moffatt \& Cookson, 1998), epilepsy (Greenberg et al., 2000), bipolar disorder (Stine et al., 1995) and Alzheimer's disease (Davies, Isles \& Wilkinson, 2005). Moreover, the rising incidence of imprintingassociated diseases in children resulting from assisted reproductive technologies (ART) is currently fervently disputed (Wilkins-Haug, 2009). In addition, it has been suggested that imprinting may also play a role in anthropometric traits such as the body mass index (BMI) (Hoggart et al., 2014). The number of imprinted genes seems to be underestimated (Maeda \& Hayashizaki, 2006), which is possibly due to the fact that the applied statistical methods deliver inconsistent results and lack power due to variable factors like heterogeneity, penetrance, family and dataset size, and imprinting-mimicking confounders such as sex-specific recombination fractions (Mukhopadhyay \& Weeks, 2003; Greenberg et al., 2010).

Imprinting can be tested by linkage analysis methods. Linkage analysis evaluates the co-segregation of genetic marker alleles together with a trait in families. Methods of linkage analysis are commonly distinguished as either being parametric or nonparametric. In parametric linkage analysis, which is also known as modelbased or LOD score analysis, a certain set of trait-model parameters is explicitly assumed for the segregation of the disease. In the simplest case of a diallelic autosomal trait locus, which is assumed throughout this paper, these parameters are the disease-allele frequency $p$ and three penetrances $f_{0}, f_{1}$, and $f_{2}$, with $f_{i}$ denoting the probability that an individual with $i$ copies of the disease allele is affected by the disease. In addition, the recombination fraction $\theta$ between marker and trait locus is modeled, or the position $x$ of the trait locus in the case of a multipoint analysis. The trait-model parameters can either be pre-specified according to results from previous segregation analyses or maximized along with the recombination fraction in a joint segregation and linkage analysis. For example, a MOD score analysis allows researchers to jointly investigate segregation and linkage (Clerget-Darpoux, Bonaïti-Pellié \& Hochez, 1986; Risch, 1984). Due to the maximization over traitmodel parameters, MOD scores are inflated when compared to LOD scores. Since the asymptotic distribution of MOD scores is unknown in the general case, $\mathrm{p}$ values for the linkage test must be obtained by simulating the distribution of the MOD score under the null hypothesis of no linkage. Our group has implemented the MOD score approach, including a routine to perform simulations under the null hypothesis of no linkage, in the GENEHUNTER-MODSCORE (GHM) software (Brugger \& Strauch, 2014; Dietter et al., 2007; Mattheisen et al., 2008; Strauch, 2003), which is based on the GENEHUNTER program (Kruglyak et al., 1996). Its application has led to the identification of a variety of genetic disease loci responsible for congenital heart defects (Flaquer et al., 2013), allergic rhinitis (Kruse et al., 2012), atopic dermatitis (Christensen et al., 2009), bipolar affective disorder been proposed as an alternative to parametric analysis. These methods promise to avoid trait-model misspecification that may occur when using simple LOD score analyses to map genes responsible for complex traits, for which the mode of inheritance, i.e. trait-model parameters in this case, is usually unknown. Nonparametric methods test if affected pedigree members have more alleles in common than would be expected by chance under the null hypothesis of no linkage. They are often considered to be 'model-free' because they do not rely on explicit assumptions as to the trait-model parameters. However, Knapp, Seuchter, and Baur (1994) have shown that, for samples of affected sib-pairs (ASPs) with the parents' phenotypes unknown or set to unknown, the nonparametric mean test is equivalent to a LOD score analysis under a recessive mode of inheritance, and the possible triangle test proposed by Holmans (1993) is equivalent to a MOD score analysis. In the possible triangle test, the genetic likelihood is expressed in terms of the probabilities $z_{0}, z_{1}, z_{2}$ that an ASP shares zero, one, or two allele(s) identical-by-descent (IBD) with restrictions to genetically possible models (Holmans, 1993). These allele-sharing probabilities can be expressed as functions of the trait-model parameters $f_{0}, f_{1}, f_{2}, p$, and $\theta$ (Suarez, Rice \& Reich, 1978), and hence, the parametric and nonparametric likelihood are identical. Furthermore, Strauch (2007) has shown that the identity of the nonparametric and parametric likelihood holds for any type of pedigree.

In linkage analysis, it is common practice to use sex-averaged genetic maps even if sex-specific differences exist. When using the Kong-and-Cox LOD score (Kong \& Cox, 1997) or the MOD score (Risch, 1984; ClergetDarpoux, Bonaïti-Pellié \& Hochez, 1986), this does not change the type I error rate and power of the linkage test, as shown by Fingerlin, Abecasis, and Boehnke (2006) and Dietter et al. (2007), respectively, but only if the ratio of available paternal and maternal genotypes equals 1 . In contrast, inflated type I error rate and reduced power is generally observed in the case of a simple parametric multipoint LOD score analysis (Daw, Thompson \& Wijsman, 2000). In summary, the direction of the deviation in the type I error rate depends on the sex-specific availability ratio of genotypes, the actual underlying sex-specific map ratio, marker distances, 
number of markers, marker information, and sample size (Sieberts \& Gudbjartsson, 2005). Therefore it is advisable to adequately model sex-specific recombination frequencies in linkage analysis. The analysis option to include sex-specific recombination frequencies is available in many linkage analysis software packages like Allegro (Gudbjartsson et al. 2000; 2005), Merlin (Abecasis et al., 2002), Superlink (Fishelson \& Geiger, 2002), and GHM (Dietter et al., 2007). In contrast to simple LOD score calculations, the power of parametric MOD score analyses is generally less affected by map misspecifications due to the maximization over all model parameters, which effectively serve as nuisance parameters in this case. This holds true for both two- and multipoint analyses. In addition, it has been shown that, especially when analyzing a mixture of different types of pedigrees, the MOD score approach outperforms other linkage methods in terms of power to identify genes with modest effect (Flaquer \& Strauch, 2012).

Genomic imprinting introduces an asymmetry between paternal and maternal marker transmission patterns and thus can lead to a substantial loss of power when not taken into account. This directly follows from the statement for standard LOD score analysis that the power to detect linkage is maximal if the analysis model corresponds to the true disease model (Clerget-Darpoux, Bonaïti-Pellié \& Hochez, 1986). Relating to linkage tests allowing for imprinting, sex differences in genetic maps and imprinting are confounded in both parametric and nonparametric linkage analyses. It is possible to model imprinting by separately maximizing parametric LOD scores over male and female recombination fractions ( $\theta_{\text {male }}$ and $\theta_{\text {female }}$, respectively) (Smalley, 1993), where nonpenetrant cases are explained by fictitious recombinations in the imprinted sex. This results in a correct (uninflated) estimate of the recombination frequency in the nonimprinted sex and an increased LOD score, compared to an analysis with a single recombination fraction for both sexes. A more straightforward approach to model imprinting is to use a four-penetrance formulation distinguishing the heterozygotes according to the parental origin of the disease allele $\mathbf{f}=\left\{f_{0}, f_{1 \text { pat }}, f_{1 \text { mat }}, f_{2}\right\}$ implemented in the programs GHM and GENEHUNTER-IMPRINTING (Strauch et al., 2000a). Conversely, it is possible to 'model' sex-specific recombination frequencies when the above-mentioned four-penetrance formulation is used in the analysis. Importantly, confounding affects both parametric and nonparametric methods through their relation as outlined by Strauch (2007). In twopoint analyses, when modeling imprinting but not accounting for sex-specific recombination frequencies, imprinting test results can be confounded, leading to an increased type I error rate of the applied test statistic. A true parent-of-origin effect in regions with sex-specific recombination frequencies can then only be declared if the nonimprinted sex has the longer genetic map and shows excess allele-sharing in the analysis (Paterson, Naimark \& Petronis, 1999). Generally, the power to detect imprinting is bounded from above by the power to detect linkage (Lemire, 2005). Using MOD scores, a parent-of-origin effect seems likely if the MOD score with four penetrances accounting for imprinting ('IMOD score') is remarkably higher than the MOD score with only three penetrances not accounting for imprinting. Our newly proposed test statistic MOBIT (see Methods section) corresponds to the difference between the two aforementioned scores: MOBIT = (IMOD score) - (MOD score). When applying sex-averaged recombination frequencies when in fact no imprinting but sex-specific recombination fractions are present, the nonimprinting MOD score is reduced due to an increased number of observed recombinations in the sex with the longer genetic map. In contrast, in an IMOD score analysis, an additional recombination in the sex with the longer genetic map is not modeled as such but the offspring is instead interpreted as a nonpenetrant carrier. This leads to an increased difference between the IMOD and the MOD score, i.e. to confounding. However, when performing multipoint analyses, the disease locus is confined between flanking markers. Hence, the possibility of double recombinations between two adjacent markers is quite low as long as the marker framework is adequately dense (Strauch et al., 2000b). In this case, the linkage analysis no longer loses information due to an increased number of recombinations in the sex having the longer genetic map since linkage to at least one side of the marker framework is preserved, and so it can be expected that the confounding vanishes. Still, in the case of a sparse marker framework and/or large sex-specific map ratios, the probability of double recombinations is non-negligible and confounding might reappear (Strauch et al., 2000b). It has been proposed that confounding is not an issue if map ratios are $<5: 1$ for LOD score analyses allowing for imprinting (Mukhopadhyay \& Weeks, 2003) or <10:1 for quantitative trait loci (QTL) LOD score analyses accommodating imprinting (Hanson et al., 2001). Furthermore, marker spacings of $<5 \mathrm{cM}$ (Vincent et al., 2006) or $<1 \mathrm{cM}$ (Wu, Shete \& Amos, 2005) have been proposed to be sufficiently dense to avoid confounding. However, there is no comprehensive and consensual answer to the question to what extent marker spacing, sex-specific map ratios, sample size, and pedigree structure influence confounding in twoand multipoint analyses. This issue is addressed in our extensive simulation study using nuclear families with two affected siblings and extended pedigrees. Three hypotheses (no linkage and no imprinting; linkage and no imprinting; linkage and imprinting) were considered in order to thoroughly investigate the performance of the likelihood-based imprinting test statistic MOBIT and the degree to which imprinting is confounded with sex-specific maps under various ratios. Further, the effect of the sample size was also investigated. In addition, power and the ability of the MOBIT to quantify imprinting were assessed. Finally, the MOBIT was also applied in a real data example on house dust mite allergy to demonstrate the applicability of the MOBIT, for 
which the statistical significance was assessed using two alternative simulation/permutation procedures for the calculation of empiric $p$ values.

Throughout this paper, a monogenic dichotomous trait is considered. It should be noted that methods also exist to map imprinted quantitative and ordinal trait loci, see e.g. Shete, Zhou, and Amos (2003) and Feng and Zhang (2008), respectively. Likewise, it is of note that a variety of nonparametric linkage-based imprinting tests have been proposed (Lemire, 2005; Liu et al., 2005; Wu, Shete \& Amos, 2005; Vincent et al., 2006). However, these methods either assume independence of parental meioses (Lemire, 2005; Liu et al., 2005; Vincent et al., 2006), are restricted to nuclear families (Wu, Shete \& Amos, 2005; Vincent et al., 2006), do not allow for sex-specific recombination fractions (Vincent et al., 2006), do not offer quantification of imprinting (Lemire, 2005; Liu et al., 2005; $\mathrm{Wu}$, Shete \& Amos, 2005), or are not explicitly designed for a maximization over the recombination fraction (Lemire, 2005; Liu et al., 2005; Vincent et al., 2006). Correlated meioses can significantly bias imprinting test results if independent parental meioses are assumed in the analysis (Vincent et al., 2006). It can be shown that parental meioses are independent for a multiplicative or a strictly recessive trait model, i.e. a recessive model without phenocopies. A negative correlation of the parental meioses is obtained for additive and dominant trait models and leads to anticonservative imprinting test results. A positive correlation is induced by recessive trait models with phenocopies as well as under- or overdominant trait models and leads to a conservative test. The already existing nonparametric methods, albeit all of them are acknowledged tests for imprinting, are not further investigated in this work due to their above-mentioned properties that render comparisons with the parametric MOBIT hardly feasible.

The paper is structured as follows. In the Methods section, the general framework of the MOBIT is introduced. Then, MOBIT analyses using either a sex-averaged or a sex-specific map for the analysis along with the ability of the MOBIT to quantify imprinting are outlined. At the end of the Methods section, the simulations and analyses of the present study are explained. This is followed by the Results section presenting the results of the simulation study and the real data example on house dust mite allergy. The paper concludes with the Discussion section and a guideline as to how linkage-based imprinting tests should be performed in practice. In addition, the paper includes an Appendix that contains proofs of the asymptotic distribution of the MOBIT, a proof of the identifiability of the marker-trait locus distance in the case of a sex-specific MOD score analysis, and details of a newly developed MOBIT permutation procedure.

\section{Methods}

\subsection{MOBIT - general framework}

Generally, in a nonparametric linkage analysis, imprinting can be assessed by looking at the allele-sharing difference between paternal and maternal meioses (Paterson, Naimark \& Petronis, 1999). To investigate parentof-origin effects in ASPs, a nonparametric test has been proposed by Knapp and Strauch (2004), which does not assume independent parental meioses. In that work, the classical Holmans' possible triangle test statistic $T_{\text {Holmans }}$ for ASPs (Holmans, 1993) is extended to the test statistic $T_{I L R}$ which includes four instead of three IBD allele-sharing probabilities by splitting up the probability $z_{1}$ of one allele IBD into two probabilities $z_{1}^{\text {pat }}$ and $z_{1}^{\text {mat }}$ according to the parental origin of the allele, such that $z_{1}=z_{1}^{\text {pat }}+z_{1}^{\text {mat }}$. Regarding the parameter space, this leads to an extension of the possible triangle to a tetrahedron which accounts for disease models with $z_{1}^{\text {pat }} \neq z_{1}^{\text {mat }}$ (see Figure 1). When analyzing ASPs with parental phenotypes set to unknown and employing a sex-averaged marker map, the parametric MOD score is equivalent to the nonparametric $T_{\text {Holmans }}$ (Knapp, Seuchter \& Baur, 1994) and the MOD score with four penetrances accounting for imprinting (IMOD score) is equivalent to the nonparametric $T_{I L R}$ (Knapp \& Strauch, 2004). Going beyond a test for linkage adaptive to imprinting, the MOD and the IMOD score can be combined to test the null hypothesis of linkage but no imprinting by evaluation of the difference of the respective maximized log-likelihood ratios with and without distinguishing the heterozygotes, which is given by 


$$
\begin{aligned}
\text { MOBIT } & =(\text { IMOD score })-(\text { MOD score }) \\
& =\log _{10} \frac{L\left(\hat{z}_{0}, \hat{z}_{1}^{\text {pat }}, \hat{z}_{1}^{\text {mat }}, \hat{z}_{2}\right)}{L\left(\frac{1}{4}, \frac{1}{4}, \frac{1}{4}, \frac{1}{4}\right)}-\log _{10} \frac{L\left(\hat{z}_{0}, \hat{z}_{1}, \hat{z}_{2}\right)}{L\left(\frac{1}{4}, \frac{1}{2}, \frac{1}{4}\right)} \\
& =\log _{10}\left(L\left(\hat{z}_{0}, \hat{z}_{1}^{\text {pat }}, \hat{z}_{1}^{\text {mat }}, \hat{z}_{2}\right)\right)-\log _{10}\left(L\left(\frac{1}{4}, \frac{1}{4}, \frac{1}{4}, \frac{1}{4}\right)\right) \\
& -\log _{10}\left(L\left(\hat{z}_{0}, \hat{z}_{1}, \hat{z}_{2}\right)\right)+\log _{10}\left(L\left(\frac{1}{4}, \frac{1}{2}, \frac{1}{4}\right)\right) \\
& =\log _{10} \frac{L\left(\hat{z}_{0}, \hat{z}_{1}^{\text {pat }}, \hat{z}_{1}^{\text {mat }}, \hat{z}_{2}\right)}{L\left(\hat{z}_{0}, \hat{z}_{1}, \hat{z}_{2}\right)}
\end{aligned}
$$
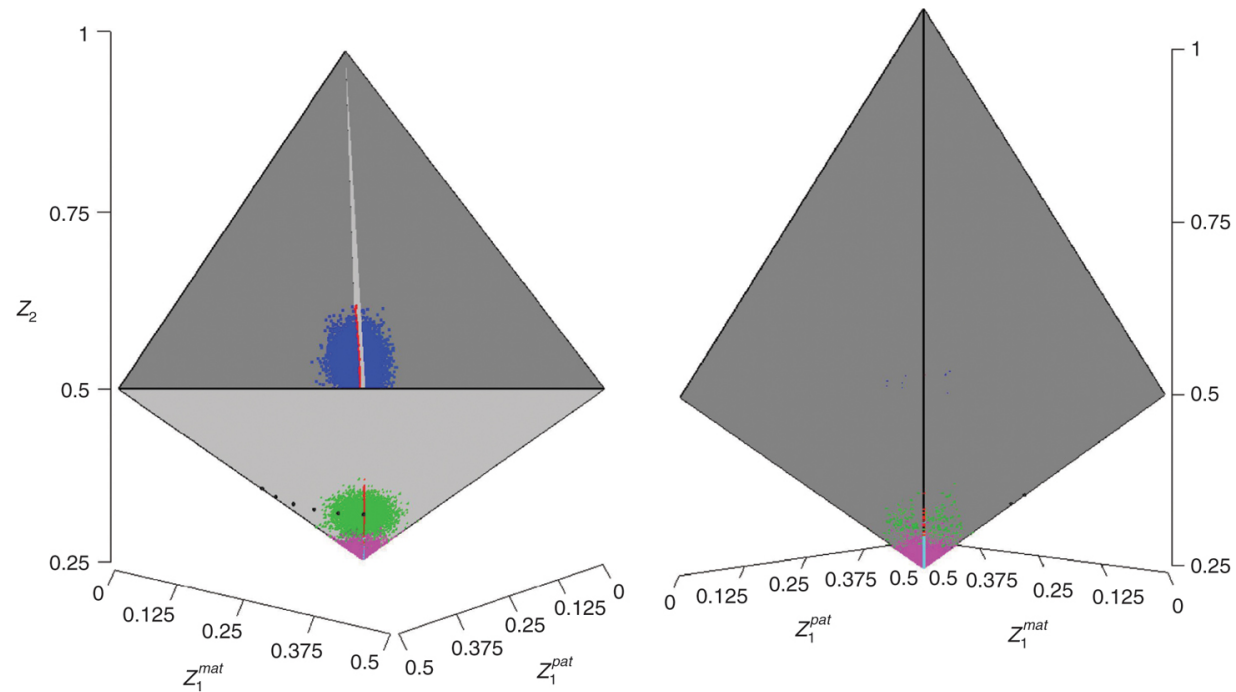

Figure 1: Tetrahedron $\mathbf{T}$ defining the set of allele-sharing probabilities $\mathbf{z}=\left(z_{0}, z_{1}^{\text {pat }}, z_{1}^{\text {mat }}, z_{2}\right)$ for affected sib-pairs (ASPs) that correspond to meaningful genetic models, as a subset of the larger set of all possible allele-sharing probabilities represented by the outer cube.

Between the left half-tetrahedron $\mathbf{T}_{\mathbf{z}_{1}^{\text {pat }}}>\mathbf{z}_{1}^{\text {mat }}$ and the right half-tetrahedron $\mathbf{T}_{\mathbf{z}_{1}^{\text {pat }}<\mathbf{z}_{1}^{\text {mat }}}$ (both in dark-grey) lies the sagittal plane (light-grey), which corresponds to the possible triangle in the nonimprinting parameter space $\left(z_{0}, z_{1}, z_{2}\right)$, dividing $\mathbf{T}$ into two halves. The left subfigure depicts $\mathbf{T}$ with the front plane (light-grey) facing towards the beholder, whereas in the right subfigure $\mathbf{T}$ is turned around, such that the nonimprinting parameter space $\left(z_{0}, z_{1}, z_{2}\right)$ can be seen as a black line dividing $\mathbf{T}$ into two halves. Black bullet points on the front triangle (also in light-grey) in the left subfigure correspond to the points in terms of allele sharing that were used to simulate the additive trait model. Hence, the black bullet in the middle of the frontern triangle corresponds to penetrances $f_{0}=0.03, f_{1}=0.13, f_{2}=0.23$, and disease allele frequency $p=0.1$. The black bullets on the left side of the front triangle (left subfigure) correspond to imprinting models, such that the farther left a bullet point lies, the higher is its corresponding imprinting index $(\mathbf{I}=\{0.2 ; 0.4 ; 0.6 ; 0.8 ; 1\})$. Green dots on the light-grey front plane represent maximum likelihood estimates (MLEs) of a MOD score analysis that takes imprinting into account (IMOD score) using 10,000 replicates of 600 ASPs and a fully informative marker with recombination fraction $\theta=0$ and the additive trait model. The corresponding nonimprinting MOD score MLEs (red dots) lie in the middle of the light-grey front plane, which corresponds to the upper edge of the possible triangle, i.e. the nonimprinting parameter space $\left(z_{0}, z_{1}, z_{2}\right)$. Pink and turquoise dots correspond to IMOD and MOD score MLEs under no linkage, respectively. With regard to the simulated recessive trait model with $f_{0}=0.05, f_{1}=0.05, f_{2}=0.9$, and disease allele frequency $p=0.2$, blue and red dots in the upper part of the left subfigure correspond to the IMOD and MOD score MLEs, respectively.

where $\hat{z}_{i=0,1,2}$ denote the maximum likelihood estimators (MLEs) restricted to the possible set of allele-sharing probabilities $\mathbf{z}$ defined by the possible triangle $(\Delta)$ for the $T_{\text {Holmans }}$ with $\mathbf{z}_{\triangle}=$ $\left\{\left(z_{0}, z_{1}, z_{2}\right): z_{1} \leq 0.5,2 \cdot z_{0} \leq z_{1}, z_{0} \geq 0, z_{1} \geq 0, z_{2} \geq 0, z_{2}=1-z_{0}-z_{1}\right\}$ and the tetrahedron (T) for the $T_{I L R}$ with $\mathbf{z}_{\mathbf{T}}=\left\{\left(z_{0}, z_{1}^{\text {pat }}, z_{1}^{\text {mat }}, z_{2}\right): z_{1}^{\text {pat }}+z_{1}^{\text {mat }} \leq 0.5, z_{0} \leq z_{1}^{\text {pat }} \leq z_{2}, z_{0} \leq z_{1}^{\text {mat }} \leq z_{2}, z_{2} \geq 0, z_{1}^{\text {pat }} \geq 0, z_{1}^{\text {mat }} \geq\right.$ $\left.0, z_{0} \geq 0, z_{0}=1-z_{2}-z_{1}^{\text {pat }}-z_{1}^{\text {mat }}\right\}$. The equivalence of the likelihoods under the null hypothesis of no linkage $L\left(\frac{1}{4}, \frac{1}{4}, \frac{1}{4}, \frac{1}{4}\right)$ and $L\left(\frac{1}{4}, \frac{1}{2}, \frac{1}{4}\right)$ is shown in Appendix A.1 of the paper. In their Discussion section, Knapp and Strauch (2004) have proposed a nonparametric imprinting test for ASPs that is (up to a factor of $2 \ln (10)$ ) identical to the MOBIT in the context of ASPs. Denoting this nonparametric imprinting test by $T_{I m p r}$, we can therefore write 


$$
\text { MOBIT }=\frac{1}{2 \ln (10)} \cdot 2 \ln \frac{L\left(\hat{z}_{0}, \hat{z}_{1}^{p a t}, \hat{z}_{1}^{m a t}, \hat{z}_{2}\right)}{L\left(\hat{z}_{0}, \hat{z}_{1}, \hat{z}_{2}\right)}=\frac{T_{I m p r}}{2 \ln (10)}
$$

The null distribution of the $T_{\text {Impr }}$ depends on the true underlying $\mathbf{z} \in H_{0}: z_{1}^{\text {pat }}=z_{1}^{\text {mat }}$ which is either an interior point of $\mathbf{T}$ or lying on its boundary. If $\mathbf{z} \in H_{0}: z_{1}^{\text {pat }}=z_{1}^{\text {mat }}$ is an interior point of $\mathbf{T}$, standard theory predicts that the asymptotic distribution of the $T_{\text {Impr }}$ is $\chi^{2}$ with $1 \mathrm{df}$, although the proximity to the boundary of $\mathbf{T}$ may affect the quality of the asymptotic approximation. If $\mathbf{z}$ is a point on the boundary of $\mathbf{T}$, the quantiles of the asymptotic distribution can be smaller than for a $\chi^{2}$ distribution with $1 \mathrm{df}$ (Knapp \& Strauch, 2004). Due to the equality, when analyzing ASPs, the same properties hold true for the MOBIT. For the point of no linkage $\mathbf{z}=\left(\frac{1}{4}, \frac{1}{4}, \frac{1}{4}, \frac{1}{4}\right)$, the MOBIT follows a mixture of distributions that includes non- $\chi^{2}$ components (see also Self and Liang (1987), case 8 , pp. 608-609). It is noteworthy that the equality of a parametric MOD score analysis with a corresponding allele-sharing-based test statistic holds in general for arbitrary pedigree structures (Strauch, 2007). However, the corresponding allele-sharing configurations have only been formulated for unilineal affected relative-pairs (ARPs), ASPs, and affected sib-triplets (ASTs) so far (Knapp, 2005; Strauch, 2007). The allele-sharing parameter spaces for larger pedigrees involve a larger number of dimensions, and the corresponding restrictions for genetically possible models are expected to have a more complicated form (Knapp, 2005; Strauch, 2007). Even for ASTs, the parameter restrictions are unknown so far, which precludes the construction of an allele-sharingbased test (Knapp, 2005). Therefore, the null distribution of the MOBIT cannot be analytically derived from an equivalent nonparametric test for larger pedigrees. However, given the equality of the nonimprinting and imprinting likelihoods under the null hypothesis of no linkage and no imprinting for any type of pedigree (Appendix A.2), the distribution is expected to be $\chi^{2}$ with $1 \mathrm{df}$, because standard likelihood ratio technique predicts that the number of degrees of freedom is equal to the difference between the maximized parameters in the numerator and the denominator, which equals $4-3=1$ in our case, which represents the case of two nested composite hypotheses (Wilks, 1938) (see also Appendix A.3).

\subsection{Sex-averaged MOBIT analysis}

Assuming sex-averaged recombination fractions, a MOD score analysis using ASPs is equivalent to $T_{\text {Holmans }}$ as shown by Knapp, Seuchter, and Baur (1994), and thus explores the same parameter space of $\mathbf{z}_{\triangle}$ defined by the possible triangle (whole triangle, light-grey sagittal plane in Figure 1). In particular, a given combination of penetrances $f_{0}, f_{1}, f_{2}$, disease allele frequency $p$, and recombination frequency $\theta$ in a parametric analysis corresponds to a certain point $\mathbf{z}$ within the possible triangle in the nonparametric context. How certain sets of parametric trait-model parameters for ASPs with or without imprinting translate into allele-sharing probabilities can be found in Knapp and Strauch (2004) and Suarez, Rice, and Reich (1978), respectively. Accordingly, the maximization of the parametric likelihood ratio over the four trait-model parameters and $\theta$ in the MOD score approach corresponds to maximizing the nonparametric allele-sharing parameters over the two-dimensional plane of the possible triangle. The IMOD score analysis, on the other hand, explores the whole tetrahedron T including points for which $z_{1}^{\text {pat }} \neq z_{1}^{\text {mat }}$ and is equivalent to the $T_{I L R}$. In this case, the parametric likelihood ratio is maximized over the disease allele frequency $p$, the recombination frequency $\theta$, and four penetrance parameters $f_{0}, f_{1 \text { pat }}, f_{1 \text { mat }}, f_{2}$ distinguishing the heterozygotes, where $f_{1 \text { pat }} \neq f_{1 \text { mat }}$ is equivalent to excess paternal or maternal allele-sharing, i.e. $z_{1}^{\text {pat }} \neq z_{1}^{\text {mat }}$. Throughout this paper, without loss of generality, $\theta_{\text {male }}$ $\leq \theta_{\text {female }}$, is assumed. If no imprinting is present, this corresponds to points in terms of allele-sharing within the left half-tetrahedron $\left(\mathbf{T}_{\mathbf{z}_{1}} \mathbf{p a t}_{\mathbf{1}} \mathbf{z}_{\mathbf{1}}^{\text {mat }}\right)$ in Figure 1 .

\subsection{Sex-specific MOBIT analysis}

The parameter space explored by the maximization of the imprinting likelihood remains unchanged when using a sex-specific marker map, i.e. it is still the whole tetrahedron $\mathbf{T}$ for ASPs. However, the maximization of the nonimprinting likelihood is now enabled to explore parameter space beyond the possible triangle, according to the sex with the longer genetic map. For a given set of trait-model parameters $f_{0}, f_{1}, f_{2}$, and $p$, the parametric nonimprinting maximization starts with $\theta_{\text {male }}=\theta_{\text {female }}=0$ at the corresponding point on the possible triangle. This holds for a sex-specific as well as for a sex-averaged genetic map. Then, in the sex-specific case, the recombination fraction is varied according to the specified genetic map ratio, i.e. $0<\theta_{\text {male }}<\theta_{\text {female }}<\frac{1}{2}$ in the analysis. This leads to points $\mathbf{z}$ within the left half-tetrahedron $\mathbf{T}_{\mathbf{z}_{1}^{\text {pat }}>\mathbf{z}_{1}^{\text {mat }}}$ outside the sagittal plane $\mathbf{z}_{\triangle}$, i.e. the 
possible triangle. The maximization over the recombination frequency continues until the point of no linkage $\theta_{\text {male }}=\theta_{\text {female }}=\frac{1}{2}$ is reached, which corresponds to $\mathbf{z}=\left(\frac{1}{4}, \frac{1}{4}, \frac{1}{4}, \frac{1}{4}\right)$. Hence, each assumed pair of recombination fractions $\theta_{\text {male }} \leq \theta_{\text {female }}$ corresponds to a point within $\mathbf{T}_{\mathbf{z}_{1}^{\text {pat }}} \geq \mathbf{z}_{1}^{\text {mat }}$. These points join to a maximization curve over the recombination fraction for a given set of disease model parameters and a fixed female/male map ratio as illustrated in Figure 2 (black line with grey diamonds). The maximization is then continued over arbitrary combinations of trait-model parameters. The range and extent of the parameter space explored by the nonimprinting maximization within $\mathbf{T}_{\mathbf{z}_{1}^{\text {pat }}}>\mathbf{z}_{1}^{\text {mat }}$ depend on (1) the assumed genetic map ratio and (2) the inter-marker distance in the case of a multipoint analysis. The more extreme the assumed map ratio, the farther reach such maximization curves into $\mathbf{T}_{\mathbf{z}_{1}^{\text {pat }}}>\mathbf{z}_{1}^{\text {mat }}$. The same holds for larger inter-marker distances. The point of maximum maternal imprinting $\mathbf{z}=\left(0, \frac{1}{2}, 0, \frac{1}{2}\right)$ is only reached with an infinite female/male map ratio. Interestingly, given a truly underlying nonimprinting disease model with $f_{1 \text { pat }}=f_{1 \text { mat }}$, a genetic map ratio less or larger than 1 , and $\theta>0$ between marker and trait locus, the respective marker-trait locus distance is identifiable in a MOD score analysis using ASPs (see Appendix B for a proof). Using a sex-specific map in the analysis should avoid confounding between genomic imprinting and sex-specific recombination fractions, such that the type I error rate of the imprinting test does not exceed its nominal level. However, the question arises, whether the power of the test can be reduced due to a confounding between the genetic position and the trait-model parameters in the maximization of the likelihoods in equation (1). That is because imprinting can be "modeled" by separately maximizing the likelihood over male and female recombination fractions $\left(\theta_{\text {male }}\right.$ and $\theta_{\text {female, }}$, respectively) (Smalley, 1993), which is already done in the nonimprinting likelihood, just as well as by using the four-penetrance formulation distinguishing the heterozygotes according to the parental origin of the disease allele $\mathbf{f}=\left\{f_{0}, f_{1 \text { pat }}, f_{1 \text { mat }}, f_{2}\right\}$ (Strauch et al., 2000a) in the imprinting likelihood. However, this effect is expected to become negligible in the case of a densely spaced marker framework.

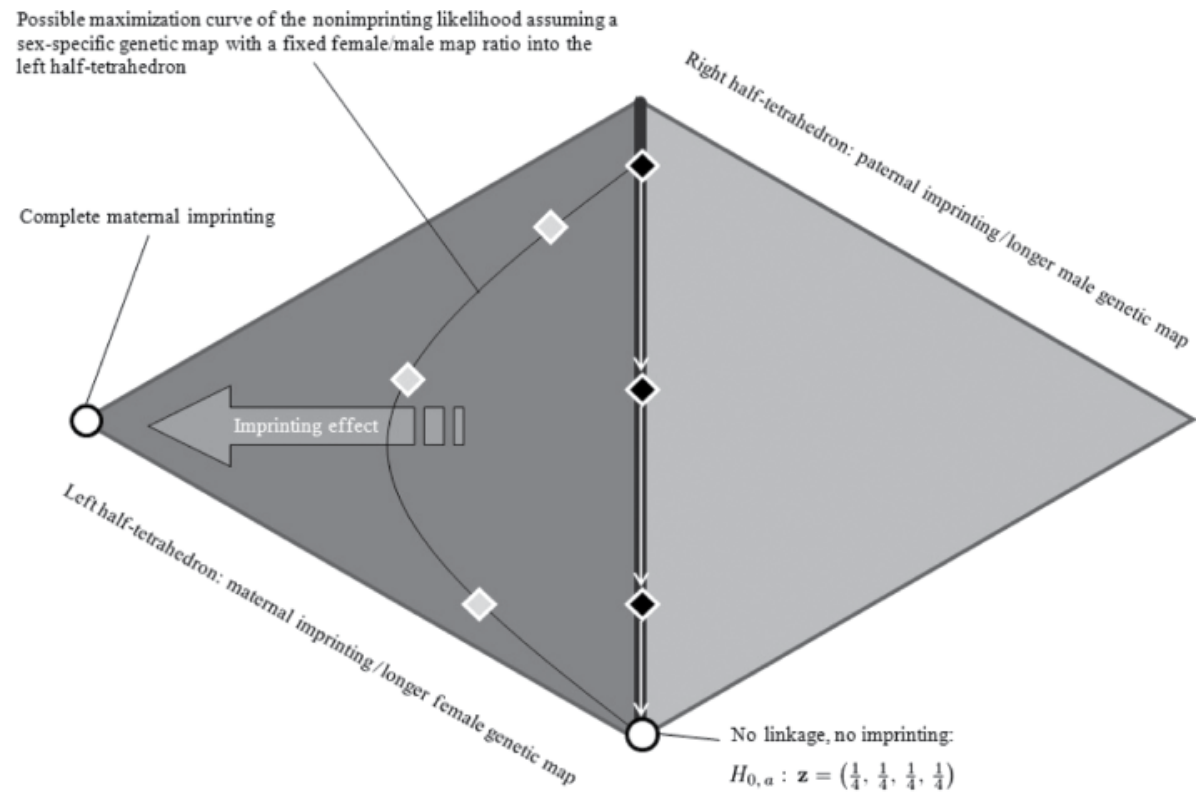


Figure 2: A graphical representation of a transversal cut through the tetrahedron $\mathbf{T}$ is shown to illustrate the nonimprinting likelihood maximization in terms of the allele-sharing parameters $\mathbf{z}=\left(\mathrm{z}_{0}, \mathrm{z}_{1}^{\mathrm{pat}}, \mathrm{z}_{1}^{\mathrm{mat}}, \mathrm{z}_{2}\right)$ within $\mathbf{T}$ in an analysis using affected sib-pairs (ASPs). The effect of a female/male genetic map ratio larger than 1 and maternal imprinting is considered. For a given set of penetrances $f_{0}, f_{1}, f_{2}$, and the disease allele frequency $p$, the sex-averaged nonimprinting maximization over the recombination frequency $\theta$ starts on the corresponding point $\mathbf{z}$ on the possible triangle (black middle line) assuming $\theta=0$ (upper black diamond). The recombination fraction is then gradually increased, leading to a curve within the possible triangle (white arrows), until $\mathbf{z}$ reaches $\left(\frac{1}{4}, \frac{1}{4}, \frac{1}{4}, \frac{1}{4}\right)$ for $\theta=\frac{1}{2}$, i.e. no linkage. In an analysis assuming a sex-specific map, the maximization over $\theta$ starts at the same initial point $\mathbf{z}$ on the possible triangle as in the sex-averaged analysis, given the same set of disease model parameters and $\theta=0$ (upper black diamond). However, the recombination fraction is now varied according to the genetic map ratio, i.e. $\frac{\theta_{\text {female }}}{\theta_{\text {male }}}>1$, which leads to explored points $\mathbf{z}$ along a curve within the left half-tetrahedron $\mathbf{T}_{\mathbf{z}_{1}^{\text {pat }}}>\mathbf{z}_{\mathbf{1}}^{\text {mat }}$ (black solid curve and grey diamonds). At $\theta=\frac{1}{2}$, $\mathbf{z}$ again reaches $\left(\frac{1}{4}, \frac{1}{4}, \frac{1}{4}, \frac{1}{4}\right)$. The extent to which a sex-specific nonimprinting maximization explores parameter space within $\mathbf{T}_{\mathbf{z}_{1}^{\text {pat }}}>\mathbf{z}_{1}^{\text {mat }}$, i.e. the outreach into the left half-tetrahedron, is increased by a larger female/male genetic map ratio. The point of complete maternal imprinting $\left(0, \frac{1}{2}, 0, \frac{1}{2}\right)$ is only reached for an infinite female/male map ratio. In addition, maternal imprinting causes $\mathbf{z}$ to be shifted farther left (large grey arrow) so that it no longer lies within the maximization scope of a sex-specific MOD score analysis not allowing for imprinting. These points are then exclusively reached by an IMOD score analysis. Yet, if $\mathbf{z}$ already lies very near to the boundary of $\mathbf{T}$ due to large differences in sex-specific recombination fractions (say, $\mathbf{z}$ corresponds to the lower grey diamond), it will hardly be shifted farther left by maternal imprinting. Hence, the sex-specific nonimprinting MOD score analysis is almost equivalent to the IMOD score analysis with respect to the explored parameter space, which leads to a lower power of the MOBIT, especially when maternal imprinting is incomplete. All these conclusions equally apply to female/male map ratios smaller than 1 and paternal imprinting.

\subsection{Quantifying genomic imprinting}

Using the MOBIT, imprinting can be quantified by looking at the imprinting index I (Strauch, 2005) calculated from the estimated penetrances at the assumed disease locus showing the highest evidence for imprinting. The imprinting index $I=\frac{f_{1} \text { pat }-f_{1 \text { mat }}}{f_{2}-f_{0}}$ equals the difference between the two heterozygote penetrances, normalized by the difference of the homozygote penetrances in order to properly take the case of a nonzero phenocopy rate or reduced penetrance into account. The question as to what extent trait-model parameters can be estimated in a MOD score analysis cannot be answered comprehensively in this paper. However, trait-model parameters can in principle be estimated by a MOD score analysis (Elston, 1989), which had been outlined in the context of the ascertainment-assumption-free method (Shute \& Ewens, 1988). However, the identifiability of the trait-model parameters $f_{0}, f_{1 \text { pat }}, f_{1 \text { mat }}, f_{2}, p$, and $\theta$ depends on the number of allele-sharing classes in the dataset. In the case of ASPs, the allele-sharing classes are $z_{0}, z_{1}^{\text {pat }}, z_{1}^{\text {mat }}$, and $z_{2}$ when taking imprinting into account. Hence, as there are only $4-1=3$ free parameters that can be estimated from ASP data, there will be many sets of $\left(f_{0}, f_{1 \text { pat }}, f_{1 \text { mat }}, f_{2}, p\right.$, and $\left.\theta \theta\right)$ that correspond to a particular estimated $\left(z_{0}, z_{1}^{\text {pat }}, z_{1}^{\text {mat }}\right.$, and $\left.z_{2}\right)$. With larger pedigrees, and hence more allele-sharing classes, the degree to which the trait-model parameters can be correctly determined should be higher. It is of note, however, that for any type of affecteds-only analysis, the absolute values of penetrances cannot be determined, because multiplication of all penetrances by the same factor does not change the result. In order to investigate the ability of our newly proposed MOBIT test to quantify imprinting, we compared the estimated imprinting degrees in terms of the imprinting index I with the simulated values when either using a sex-specific or a sex-averaged map in the analysis.

\subsection{Simulation and analysis}

The families under study were samples of either affected sib-pairs (ASPs) or extended pedigrees with three generations (3-G) (see Figure 3). A diallelic disease locus causing a dichotomous trait, with parameters that should reflect the characteristics of complex disorders, was chosen for the simulations. In order to ensure that the power to detect imprinting is sufficiently high with replicates generated under the selected parameter set and that the computations are still feasible, power calculations for the linkage test with ASPs were done using the $R$ package powerpkg (Weeks, 2010) under various parameter sets prior to performing the simulations. The parental trait phenotypes were set to unknown, and a fully informative marker in complete linkage, i.e. $\theta=0$, with the disease locus was simulated. An additive single-locus disease model with penetrances $\left\{f_{0}, f_{1}, f_{2}\right\}=$ $\{0.03,0.13,0.23\}$ and disease allele frequency $p=0.1$ was chosen, which leads to a power to detect linkage of approximately $80 \%$ using a sample size of 600 ASP families (type I error rate: $10^{-4}$ ). The respective sample sizes for the $3-G$ pedigrees were derived by initial test simulations. In particular, given $3-G$ pedigrees, sample size, a fully informative marker, and a type I error rate of $10^{-4}$, the critical value for the linkage test was determined under the null hypothesis of no linkage, and power was assessed by simulating completely linked replicates. 
This led to a sample size of $653-G$ pedigrees for the additive trait model. To evaluate the effect of sample size for confounding, we additionally analyzed samples consisting of 400 ASPs and 453 -G pedigrees, which corresponds to a power of $50 \%$ to detect linkage. It is of note that the additive trait model lies on the boundary of the parameter space of ASPs, which affects the null distribution of the MOBIT. Hence, a recessive trait model with $p=0.2$ and $\left\{f_{0}, f_{1}, f_{2}\right\}=\{0.05,0.05,0.9\}$, which lies in the interior of the parameter space of ASPs, was also considered to numerically verify the $\chi_{1}^{2}$ distribution of the MOBIT in the case of no boundary condition. The sample size used for the simulations for the recessive trait model was chosen to be the same as for the additive trait model. This way, the degrees of confounding of the imprinting tests are based on the same number of meioses for both trait models and can thus fairly be compared.

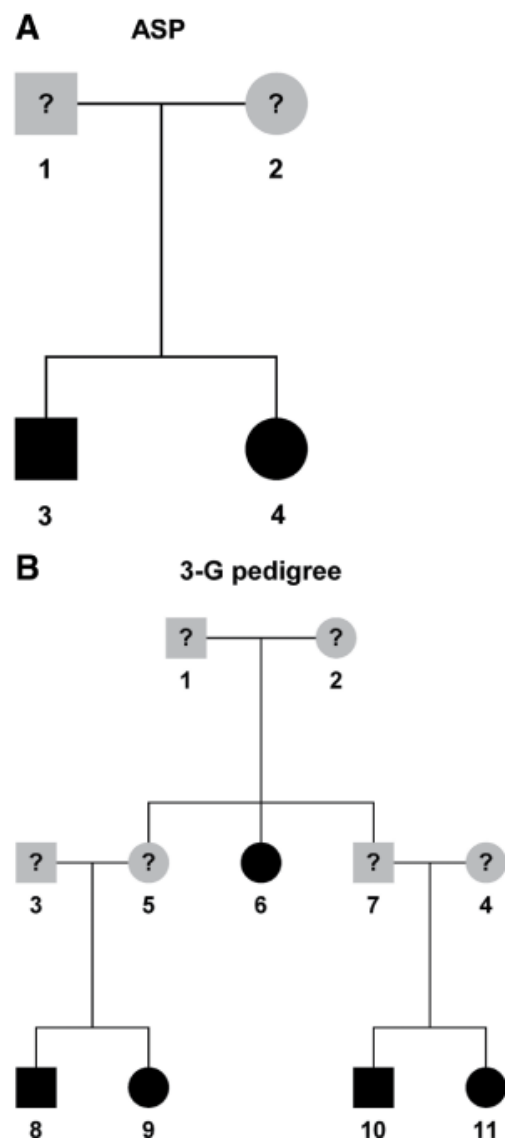

Figure 3: (A) Affected sib-pair (ASP) (B) Three-generation pedigree (3-G).

Pedigrees used for the simulations. ASP: affected sib-pair; 3-G: three-generation pedigree; ?: unknown phenotype; filled symbols: affected; empty symbols: unaffected.

Generation of genotype data with or without imprinting effects and conditional on affected offspring (with parental phenotypes assumed to be unknown) were either carried out by SLINK (Ott, 1989; Schäffer et al., 2011; Weeks et al., 1990) or by its imprinting extension SLINK Imprinting (Shete \& Zhou, 2005). The simulation algorithm calculates the probability distribution of genotypes $\mathbf{g}=g_{1}, g_{2}, \ldots, g_{n}$ conditional on the phenotype values $\mathbf{x}=x_{1}, x_{2}, \ldots, x_{n}$ of $n$ family members in a step-wise manner until all members have been assigned a genotype, each conditional on all phenotypes and the set of genotypes assigned before to other family members: $P(\mathbf{g} \mid \mathbf{x})=P\left(g_{1} \mid \mathbf{x}\right) P\left(g_{2} \mid g_{1}, \mathbf{x}\right) P\left(g_{3} \mid g_{1}, g_{2}, \mathbf{x}\right) \ldots$ The calculation time of this algorithm increases linearly with additional family members, but exponentially with the number of markers. In order to avoid prohibitive computation times when simulating several markers, a two-step algorithm developed by Lemire (2006) was employed, which exploits the ability of conditional simulations by SLINK or SLINK Imprinting, respectively, and uses a gene dropping algorithm implemented in the SLINK utility program SUP (Lemire, 2006; Schäffer et al., 2011) to quickly generate a large number of markers. This procedure starts with the generation of the disease locus genotypes and trait values by SLINK or SLINK Imprinting, respectively, where a fully informative marker in linkage equilibrium (LE) with the disease locus and $\theta=0$ is simulated to mark the path of inheritance of the disease; it is therefore called the 'descent marker'. Using SUP, it is then possible to simulate single marker alleles or haplotypes for several markers along the generations allowing for sex-specific recombination frequencies. Four scenarios were simulated: (1) a fully informative microsatellite marker with $\theta=0$ between the marker and the trait locus; (2) a fully informative microsatellite marker with two nonzero genetic distances to the disease locus; (3) four microsatellites with four equifrequent alleles corresponding to a mean heterozygosity (HET) of 0.75, 
with the disease locus placed halfway between marker 2 and 3 at various genetic distances; (4) a typical array of 40 diallelic single-nucleotide polymorphisms (SNPs), each SNP with a minor allele frequency (MAF) of 0.15 corresponding to a mean HET of 0.25 , spaced $0.32 \mathrm{cM}$ from each other and the disease locus placed halfway between SNPs 20 and 21. The genetic distances between the marker(s) and the disease locus in the single and four-marker scenarios (2) and (3) were 0.5 and $5 \mathrm{cM}$, respectively. The former distance implies a marker spacing of $1 \mathrm{cM}$, which may be used for fine-mapping, and the latter distance corresponds to a sparse marker spacing of $10 \mathrm{cM}$. Besides sex-equal recombination frequencies, two female/male map ratios were assumed. A map ratio of 7:3 was simulated as a possible value for an imprinted region like the one on chromosome 11p13 around the WT1 gene showing a ratio of about 2.1:1 (Pàldi, Gyapay \& Jami, 1995), whereas a map ratio of about 9:1 can be found in the pseudoautosomal regions PAR1 and PAR2 on the X and Y chromosome (Flaquer, Fischer \& Wienker, 2009; Matise et al., 2007). To evaluate the size and the power of the MOBIT, simulations under three hypotheses were analyzed: $H_{0, a}$ : no linkage, no imprinting, $H_{0, b}$ : linkage, no imprinting, and $H_{1}$ : linkage, imprinting. For the latter, five degrees of maternal imprinting were assumed, corresponding to imprinting indices of $\mathbf{I}=\{0.2 ; 0.4 ; 0.6 ; 0.8 ; 1\}$ as defined by Strauch (2005) and given above. Additionally, paternal imprinting, corresponding to $\mathbf{I}=\{-0.2 ;-0.4 ;-0.6 ;-0.8 ;-1\}$, was also considered, but only for the scenarios with $5 \mathrm{cM}$ between marker and trait locus. This is because these scenarios correspond to the case in which imprinting occurs in the sex with the shorter genetic map, so that the impact on power due to sex-specific recombination fractions and confounding should be especially relevant in the case of large marker-trait locus distances. In the case of the simulated additive trait model, the penetrances were $f_{0}=0.03$ and $f_{2}=0.23$ and the average of $f_{1 \text { pat }}$ and $f_{1 \text { mat }}$ was 0.13 , corresponding to the nonimprinting case. An overview of the simulation scenarios can be found in Table 1. It should be noted that the concepts of dominance, recessivity, or multiplicativity do not make sense under imprinting conditions (Strauch et al., 2000a). More specifically, a recessive trait model like the one we used for the simulations has no corresponding 'recessive' imprinting model within the diamond of inheritance (DOI) (Strauch, 2005). Instead, with an increasing degree of imprinting, the model approaches the horizontal axis of the DOI $\left(\frac{f_{1, \text { pat }}+f_{1, \text { mat }}}{2}=\frac{f_{0}+f_{2}}{2}\right)$, which is closer to additive than dominant or recessive models. A similar relation holds for the corresponding points in terms of allele-sharing. Therefore, simulations under $H_{1}$ were only done for the additive disease model, which lies on the horizontal axis of the DOI, and, for reasons of conciseness, only for the twopoint scenarios (1) and (2) as well as the SNPs scenario (4). The number of replicates was set to 10,000 , except for the scenarios under $H_{1}$ with 5,000 replicates.

Table 1: Overview of the simulated scenarios to investigate confounding between sex-specific recombination fractions and genomic imprinting. SNP: single nucleotide polymorphism; ASP: affected sib-pair; 3-G pedigree: three-generation pedigree. *Only for the twopoint scenarios with $5 \mathrm{cM}$ marker-trait locus distance.

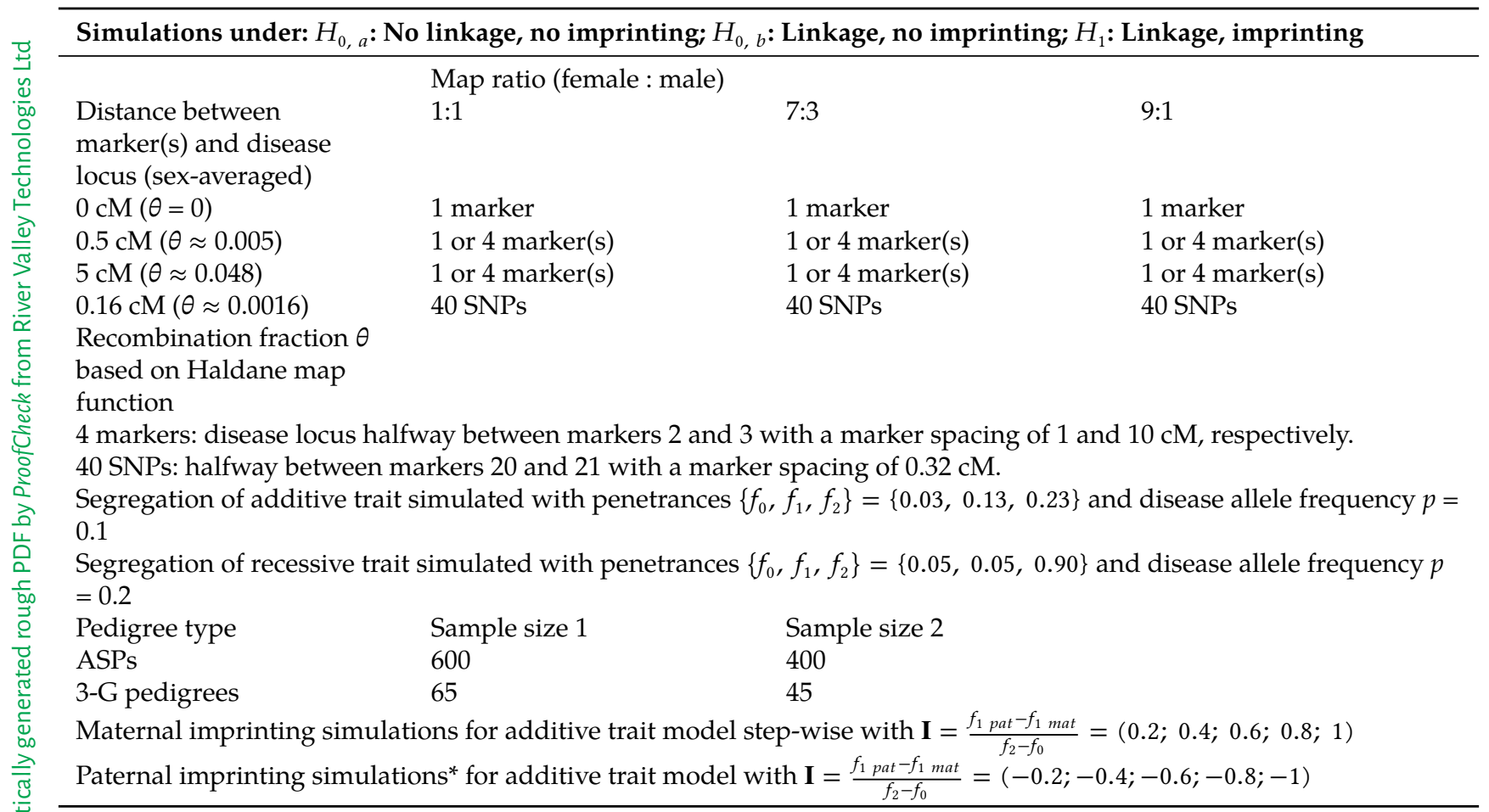

Each simulated replicate was subsequently analyzed by the GHM program, in which both the MOD score (option 'imprinting off') and the IMOD score (option 'imprinting on') were calculated in a single program 
run. Technically, the calculation of the MOBIT is realized by applying the GHM option 'modcalc global', by which the maximum of the LOD score over all assumed disease locus positions along the marker map is determined for each trait model, and this maximum is then maximized over different trait models. Further, the options 'maximization dense', 'penetrance restriction off', and 'allfreq restriction off' were used in the analysis. In terms of allele-sharing probabilities, the MOBIT compares the MLE of $\mathbf{z}_{\mathbf{T}}$ (imprinting) with the one of $\mathbf{z}_{\triangle}$ (no imprinting) for ASPs, allowing for different disease locus positions in the numerator and the denominator of the likelihood ratio in equation (1) at which the MLE is calculated. Regarding the twopoint scenarios, the MOBIT was evaluated at the marker locus and at 100 equally spaced genetic positions lying up to $100 \mathrm{cM}$ away from the marker locus. In the multipoint scenarios, the MOBIT was calculated with the putative trait locus positioned directly at the markers and halfway between them. The maximization was done over the aforementioned set of genetic positions $x$ of the putative trait locus or $\theta$ in the case of a twopoint scenario as well as over all trait-model parameters, i.e. the disease allele frequency $p$ and the penetrances $f_{0}, f_{1}, f_{2}$ without imprinting or $f_{0}, f_{1 \text { pat }}, f_{1 \text { mat }}, f_{2}$ with imprinting taken into account. All replicates were analyzed using a sex-averaged map and a sex-specific map with the same genetic map ratio as used for the simulation. The imprinting test statistic $M O B I T=(I M O D$ score $)-(M O D$ score $)$ was calculated and its empiric distributions under all three hypotheses were determined assuming either a sex-averaged or a sex-specific map for the analysis. Finally, the $95 \%$ and $99 \%$ quantiles of the empiric MOBIT distributions under the $H_{0, a}$ and $H_{0, b}$ hypotheses were obtained. Empiric quantiles were calculated according to the formula $\mathrm{p}=\frac{k}{n}, n$ being the total number of replicates and $k$ the number of ordered replicates. The test statistic of the $k$-th ordered replicate, which corresponds to a $\mathrm{p}$ value with 1 - p equal to $95 \%$ or $99 \%$, defines the respective empiric quantile. Type I error rates using a nominal 1\% and 5\% significance level were calculated using the assumed asymptotic distribution of $\chi^{2}$ with $1 \mathrm{df}$. Power was measured as the proportion of replicates simulated under $H_{1}$ : linkage and imprinting that exceeded the respective empiric $95 \%$ quantile determined under $\mathrm{H}_{0, b}$, thus ruling out inflated type I error rates due to confounding of imprinting with sex-specific recombination frequencies. In addition, we assessed the performance of the MOBIT with respect to its ability to correctly estimate the imprinting index in the scenarios used for the power calculations. We also looked for differences in estimation accuracy between sex-averaged and sex-specific analyses, maternal and paternal imprinting, and the two pedigree types.

\subsection{Real data example on house dust mite allergy}

Due to the dependence of the asymptotic properties of the MOBIT on the truly underlying point in terms of allele-sharing within the corresponding parameter space, $\mathrm{p}$ values obtained from a $\chi_{1}^{2}$ distribution might lead to false positive or false negative test results in practice. Therefore, one might simulate the MOBIT distribution under the null hypothesis of linkage, but no imprinting, such that the best-fitting nonimprinting model, including the recombination fraction or the genetic position of the disease, is used for the simulation of genotype data (method bfnm), which can be done ab initio using the software packages SLINK (Ott, 1989; Schäffer et al., 2011; Weeks et al., 1990) and SUP (Lemire, 2006; Schäffer et al., 2011). Method bfnm is similar to the simulation approach of the above-described main simulation study using a truly underlying hypothesis, which, however, is unknown in the general case. An alternative approach, similar to the methods proposed by Dong et al. (2005) and Whittaker et al. (2003), might be to obtain a $p$ value by the use of a permutation procedure based on the randomization of the origin of parental alleles in offspring of every nuclear family within a given pedigree (method perm). Hence, we have developed a permutation procedure for the MOBIT to obtain empiric $p$ values and implemented it in the GHM software package. Such a procedure effectively isolates the imprinting effect from overall evidence of linkage (Dong et al., 2005). In contrast to method bfnm, the null hypothesis of the newly implemented MOBIT-based perm procedure corresponds to an imprinting effect with expectation value 0 , conditional on the linkage information of the real dataset. As a consequence, application of method perm can lead to a completely different sample space compared to method bfnm, and can hence lead to different quantiles and $p$ values (see also Appendix C). Importantly, the power of a permutation test is restricted by the sample size, which needs to be sufficiently large to obtain a fine-grained permutation distribution. More details as to the permutation procedure can be found in Appendix C. To demonstrate the applicability of the MOBIT in practice, we reanalyzed a subset of the house dust mite allergy dataset, which originally comprises pedigrees from England, Germany, Italy, and Portugal (a detailed description of the dataset can be found in Kurz et al. (2000)). The reanalyzed subset consisted of the English families, which showed a promising result with a maximum imprinting MOD score of 4.76 near the marker locus D8S511 on chromosome 8 when a model that implies complete maternal imprinting was used in the analysis (Strauch et al., 2000a). The English subset consisted of 19 families with 125 individuals, including 7 families with 2 affected sibs, 3 families with 3 affected sibs, 5 families with 4 affected sibs, and 4 extended pedigrees. One hundred fifty microsatellite markers were typed on chromosomes 1-21, with an average spacing of $10 \mathrm{cM}$ at each candidate region. With regard to the sparseness of the marker 
map, we used sex-specific genetic distances according to the Généthon map (Dib et al., 1996) in the analysis. As a first step, nonimprinting MOD scores and IMOD scores were calculated in a single run using the GHM software with options 'modcalc global', 'maximization dense', 'penetrance restriction off', and 'allfreq restriction off', which corresponds to a thorough evaluation of many sets of trait-model parameters in the analysis. MOD scores were calculated at all marker loci and at 9 equally spaced positions between them. Because evidence for linkage should be coupled with evidence for imprinting, we decided to interpret a significant MOBIT result to be meaningful when the corresponding IMOD score was higher than 4 . For the most promising result, we obtained an empiric $\mathrm{p}$ value for the MOBIT using both methods $\mathrm{bfnm}$ and perm. With respect to method $\mathrm{bfnm}$, replicates under the null hypothesis of linkage, but no imprinting, were generated, such that the best-fitting nonimprinting model was used for the simulation of genotype data for 1,000 replicates using SLINK (Ott, 1989; Schäffer et al., 2011; Weeks et al., 1990) and SUP (Lemire, 2006; Schäffer et al., 2011). As for method perm, we generated 1,000 replicates using the newly developed permutation procedure. The corresponding empiric $p$ value for the real dataset was calculated according to the formula $\mathrm{p}=\frac{k}{n}, n$ being the total number of replicates and $k$ the number of ordered replicates showing a MOBIT that was higher or equal to the one obtained from the real dataset.

\section{Results}

\section{1 $H_{0, a}$ : No linkage, no imprinting}

The empiric $95 \%$ and $99 \%$ quantiles as well as the corresponding type I error rates assuming a $\chi^{2}$ distribution with $1 \mathrm{df}$ of the simulations under $H_{0, a}$ : no linkage and no imprinting can be found in Table 2 (ASPs) and Table 3 (3-G pedigrees). Multipoint scenarios often showed slightly higher quantiles due to an increased effective number of tests compared to twopoint scenarios. It is of note that MOBIT quantiles for ASPs under the null hypothesis of no linkage and no imprinting are expected to show lower quantiles than the assumed $\chi^{2}$ distribution with $1 \mathrm{df}$ (Knapp \& Strauch, 2004), which is the distribution of the MOBIT under $H_{0, b}$ : linkage, but no imprinting. Due to the absence of linkage, the results did not differ between the additive and recessive trait model. The results were similar for the investigated two sample sizes. If not stated otherwise, conclusions drawn from empiric quantiles also apply to the corresponding type I error rates.

Table 2: Empiric quantiles (95\% and 99\%) and type I error rates (nominal $\alpha=0.05 ; 0.01)$ of the simulated ASP scenarios

under $H_{0, a}$ : No linkage, no imprinting.

\begin{tabular}{|c|c|c|c|c|c|c|c|c|c|c|c|c|c|}
\hline \multirow{3}{*}{$\begin{array}{l}\text { Trait model } \\
\text { Sample size } \\
\text { Map ratio }\end{array}$} & & \multicolumn{6}{|c|}{ Additive } & \multicolumn{6}{|c|}{ Recessive } \\
\hline & & \multicolumn{3}{|r|}{400} & \multicolumn{3}{|r|}{600} & \multicolumn{3}{|r|}{400} & \multicolumn{3}{|r|}{600} \\
\hline & & 1:1 & $7: 3$ & 9:1 & 1:1 & $7: 3$ & 9:1 & 1:1 & $7: 3$ & 9:1 & 1:1 & $7: 3$ & 9:1 \\
\hline \multicolumn{14}{|c|}{ Analysis using a sex-averaged map } \\
\hline \multirow[t]{4}{*}{ One marker } & $95 \%$ & 0.4632 & 0.4789 & 0.4711 & 0.4514 & 0.4693 & 0.4646 & 0.4615 & 0.449 & 0.443 & 0.4574 & 0.4504 & 0.4514 \\
\hline & $99 \%$ & 0.87 & 0.9115 & 0.8596 & 0.8987 & 0.8727 & 0.874 & 0.9155 & 0.8625 & 0.8702 & 0.8911 & 0.8937 & 0.8903 \\
\hline & $5 \%$ & 0.0122 & 0.013 & 0.0113 & 0.0128 & 0.0121 & 0.0113 & 0.0123 & 0.0109 & 0.0114 & 0.0118 & 0.0115 & 0.0118 \\
\hline & $1 \%$ & 0.0016 & 0.0018 & 0.0011 & 0.0017 & 0.0009 & 0.001 & 0.0017 & 0.0013 & 0.0015 & 0.0015 & 0.0012 & 0.0014 \\
\hline \multirow{4}{*}{$\begin{array}{l}\text { Four } \\
\text { markers, } 1 \\
\text { cM }\end{array}$} & $95 \%$ & 0.5224 & 0.534 & 0.5458 & 0.5462 & 0.5326 & 0.5276 & 0.5328 & 0.5398 & 0.5407 & 0.5355 & 0.5108 & 0.5111 \\
\hline & $99 \%$ & 0.9569 & 0.9616 & 0.9747 & 0.9575 & 0.9584 & 0.9803 & 0.9374 & 0.9757 & 0.982 & 0.9602 & 0.9634 & 0.9433 \\
\hline & $5 \%$ & 0.0156 & 0.0159 & 0.0165 & 0.0195 & 0.0162 & 0.0154 & 0.0148 & 0.0168 & 0.0174 & 0.0167 & 0.0157 & 0.0146 \\
\hline & $1 \%$ & 0.0015 & 0.0016 & 0.0021 & 0.0022 & 0.0022 & 0.0018 & 0.0017 & 0.0026 & 0.0019 & 0.0021 & 0.0018 & 0.0014 \\
\hline \multirow{4}{*}{$\begin{array}{l}\text { Four } \\
\text { markers, } 10 \\
\text { cM }\end{array}$} & $95 \%$ & 0.6394 & 0.6355 & 0.6346 & 0.6278 & 0.6612 & 0.6249 & 0.6615 & 0.6373 & 0.6308 & 0.6379 & 0.6302 & 0.6326 \\
\hline & $99 \%$ & .1121 & 1.1074 & 1.134 & 1.0771 & 1.1162 & 1.113 & 1.1649 & 1.1273 & 1.0802 & 1.0901 & 1.0831 & 1.1363 \\
\hline & $5 \%$ & 0.0258 & 0.0259 & 0.0262 & 0.0248 & 0.0259 & 0.0256 & 0.0277 & 0.0264 & 0.0245 & 0.0267 & 0.0243 & 0.0271 \\
\hline & $1 \%$ & 0.0033 & 0.0032 & 0.0042 & 0.003 & 0.0041 & 0.0034 & 0.0038 & 0.004 & 0.0027 & 0.0034 & 0.0027 & 0.0035 \\
\hline \multirow[t]{4}{*}{40 SNPs } & $95 \%$ & 0.6688 & 0.6352 & 0.6536 & 0.6674 & 0.6539 & 0.6399 & 0.6361 & 0.6535 & 0.6457 & 0.6378 & 0.6357 & 0.6347 \\
\hline & $99 \%$ & 1.1135 & 1.1125 & 1.1329 & 1.1439 & 1.1532 & 1.1963 & 1.0978 & 1.1101 & 1.1287 & 1.0549 & 1.1109 & 1.1004 \\
\hline & $5 \%$ & 0.027 & 0.0273 & 0.0261 & 0.028 & 0.0269 & 0.028 & 0.0247 & 0.027 & 0.0267 & 0.0235 & 0.0247 & 0.0258 \\
\hline & $1 \%$ & 0.0034 & 0.0038 & 0.0043 & 0.0032 & 0.0033 & 0.0046 & 0.0026 & 0.0037 & 0.0043 & 0.0035 & 0.0031 & 0.0033 \\
\hline
\end{tabular}




\begin{tabular}{lrllllllllllll}
\hline \multicolumn{1}{l}{ Analysis using the sex-specific map as employed for the simulation } \\
\hline One marker & $95 \%$ & 0.4785 & 0.2678 & 0.3063 & 0.4546 & 0.3115 & 0.2847 & 0.459 & 0.2708 & 0.3129 & 0.456 & 0.3142 & 0.315 \\
& $99 \%$ & 0.9041 & 0.6778 & 0.7162 & 0.8863 & 0.6831 & 0.6896 & 0.869 & 0.6309 & 0.6989 & 0.8895 & 0.7328 & 0.7305 \\
& $5 \%$ & 0.0128 & 0.0056 & 0.0062 & 0.0118 & 0.0056 & 0.0058 & 0.011 & 0.0052 & 0.005 & 0.0125 & 0.0065 & 0.0071 \\
& $1 \%$ & 0.0014 & 0.0008 & 0.0005 & 0.0017 & 0.0008 & 0.0008 & 0.0011 & 0.0009 & 0.0004 & 0.0015 & 0.0006 & 0.0005 \\
Four & $95 \%$ & 0.526 & 0.524 & 0.5168 & 0.5329 & 0.5268 & 0.508 & 0.524 & 0.5307 & 0.5034 & 0.529 & 0.5322 & 0.521 \\
markers, 1 & & & & & & & & & & & & & \\
cM & & & & & & & & & & & & & \\
& $99 \%$ & 0.9714 & 0.9141 & 0.9531 & 0.968 & 0.9425 & 0.9186 & 0.996 & 0.9753 & 0.956 & 0.9858 & 0.9949 & 1.0189 \\
& $5 \%$ & 0.0167 & 0.0138 & 0.0172 & 0.0166 & 0.0162 & 0.0143 & 0.018 & 0.0167 & 0.0161 & 0.0161 & 0.0164 & 0.017 \\
& $1 \%$ & 0.0029 & 0.0014 & 0.0012 & 0.0015 & 0.0012 & 0.0011 & 0.0025 & 0.0023 & 0.0013 & 0.0013 & 0.0022 & 0.0023 \\
Four & $95 \%$ & 0.6305 & 0.6315 & 0.6571 & 0.6575 & 0.6509 & 0.6647 & 0.6742 & 0.6682 & 0.6409 & 0.6396 & 0.6709 & 0.6622 \\
markers, 10 & & & & & & & & & & & & \\
cM & & & & & & & & & & & & & \\
& $99 \%$ & 1.0919 & 1.1001 & 1.1292 & 1.1057 & 1.1789 & 1.1542 & 1.1419 & 1.1411 & 1.1412 & 1.1343 & 1.1374 & 1.1544 \\
& $5 \%$ & 0.0242 & 0.0251 & 0.0277 & 0.0271 & 0.0252 & 0.0281 & 0.0286 & 0.0285 & 0.0256 & 0.0259 & 0.0282 & 0.0271 \\
& $1 \%$ & 0.003 & 0.0027 & 0.0037 & 0.0034 & 0.0046 & 0.0039 & 0.0035 & 0.0034 & 0.0036 & 0.0033 & 0.0038 & 0.0042 \\
40 SNPs & $95 \%$ & 0.6517 & 0.6351 & 0.6476 & 0.6564 & 0.66 & 0.6309 & 0.6431 & 0.6248 & 0.6632 & 0.6212 & 0.6439 & 0.6483 \\
& $99 \%$ & 1.1255 & 1.1045 & 1.1275 & 1.1396 & 1.1107 & 1.1787 & 1.156 & 1.1142 & 1.1284 & 1.0623 & 1.0784 & 1.1038 \\
& $5 \%$ & 0.0271 & 0.0248 & 0.0285 & 0.0278 & 0.0265 & 0.0267 & 0.0261 & 0.0239 & 0.0289 & 0.0242 & 0.0255 & 0.027 \\
& $1 \%$ & 0.0038 & 0.0034 & 0.0041 & 0.0036 & 0.0032 & 0.005 & 0.0026 & 0.0029 & 0.0035 & 0.0029 & 0.0029 & 0.003 \\
\hline
\end{tabular}

Values in cM correspond to sex-averaged inter-marker distances. 95\% (99\%) quantile of a $\chi^{2}$ distribution with $1 \mathrm{df}$, divided by $2 \log (10)$ : 0.8342 (1.4407). For more details see Table 1.

Table 3: Empiric quantiles (95\% and 99\%) and type I error rates (nominal $\alpha=0.05 ; 0.01$ ) of the simulated 3-G pedigree scenarios under $H_{0, a}$ : No linkage, no imprinting.

\begin{tabular}{|c|c|c|c|c|c|c|c|c|c|c|c|c|c|}
\hline \multicolumn{14}{|c|}{ 3-G pedigrees } \\
\hline Trait model & & \multicolumn{6}{|c|}{ additive } & \multicolumn{6}{|c|}{ recessive } \\
\hline \multicolumn{2}{|l|}{ Sample size } & & & 45 & & & 65 & & & 45 & & & 65 \\
\hline \multicolumn{2}{|c|}{$\begin{array}{l}\text { Map ratio } \\
\text { Analysis using a sex- }\end{array}$} & $\begin{array}{c}1: 1 \\
\text { veraged }\end{array}$ & $\begin{array}{l}7: 3 \\
\text { map }\end{array}$ & 9:1 & $1: 1$ & $7: 3$ & 9:1 & 1:1 & $7: 3$ & 9:1 & 1:1 & $7: 3$ & 9:1 \\
\hline \multirow[t]{4}{*}{ One marker } & $95 \%$ & 1.1784 & 1.1363 & 1.1458 & 1.2075 & 1.2013 & 1.168 & 1.1842 & 1.1924 & 1.1754 & 1.1781 & 1.1712 & 1.2015 \\
\hline & $99 \%$ & 1.8483 & 1.7681 & 1.8312 & 1.9187 & 1.8871 & 1.8453 & 1.8695 & 1.8176 & 1.8187 & 1.812 & 1.7667 & 1.8368 \\
\hline & $5 \%$ & 0.1144 & 0.1108 & 0.1113 & 0.1154 & 0.1192 & 0.1164 & 0.1181 & 0.1176 & 0.1138 & 0.1189 & 0.1179 & 0.12 \\
\hline & $1 \%$ & 0.0256 & 0.0231 & 0.0258 & 0.0299 & 0.0285 & 0.0249 & 0.0274 & 0.0269 & 0.0252 & 0.0251 & 0.0243 & 0.0286 \\
\hline \multirow{4}{*}{$\begin{array}{l}\text { Four } \\
\text { markers, } 1 \\
\text { cM }\end{array}$} & $95 \%$ & 1.0853 & 1.0879 & 1.0692 & 1.1269 & 1.1052 & 1.092 & 1.1044 & 1.1051 & 1.0927 & 1.1092 & 1.1481 & 1.1144 \\
\hline & $99 \%$ & 1.7545 & 1.7816 & 1.7622 & 1.76 & 1.7934 & 1.8055 & 1.7989 & 1.7868 & 1.7949 & 1.7637 & 1.7961 & 1.8015 \\
\hline & $5 \%$ & 0.0958 & 0.0951 & 0.0935 & 0.1014 & 0.1003 & 0.0975 & 0.0965 & 0.0979 & 0.0929 & 0.1001 & 0.1025 & 0.0995 \\
\hline & $1 \%$ & 0.021 & 0.0206 & 0.0194 & 0.022 & 0.0229 & 0.0231 & 0.0223 & 0.0222 & 0.0214 & 0.0235 & 0.0257 & 0.0238 \\
\hline \multirow{4}{*}{$\begin{array}{l}\text { Four } \\
\text { markers, } 10 \\
\text { cM }\end{array}$} & $95 \%$ & 1.2165 & 1.2294 & 1.2033 & 1.2327 & 1.2276 & 1.2369 & 1.2221 & 1.2127 & 1.2046 & 1.2183 & 1.228 & 1.2448 \\
\hline & $99 \%$ & 1.8925 & 1.88 & 1.813 & 1.8845 & 1.9003 & 1.9725 & 1.8617 & 1.8686 & 1.9218 & 1.9072 & 1.8998 & 1.8774 \\
\hline & $5 \%$ & 0.1249 & 0.1212 & 0.1188 & 0.1227 & 0.1248 & 0.1293 & 0.1244 & 0.1188 & 0.1212 & 0.1256 & 0.1282 & 0.1251 \\
\hline & $1 \%$ & 0.0316 & 0.0318 & 0.0279 & 0.0301 & 0.0296 & 0.0323 & 0.0286 & 0.029 & 0.0285 & 0.028 & 0.0295 & 0.0291 \\
\hline \multirow[t]{4}{*}{40 SNPs } & $95 \%$ & 1.2651 & 1.227 & 1.2115 & 1.232 & 1.2571 & 1.2922 & 1.2072 & 1.2255 & 1.2246 & 1.293 & 1.2674 & 1.2696 \\
\hline & $99 \%$ & 1.9924 & 1.9454 & 1.9114 & 1.8822 & 1.9504 & 2.0957 & 1.9077 & 1.9138 & 1.9407 & 1.9018 & 1.9718 & 1.8923 \\
\hline & $5 \%$ & 0.1311 & 0.1183 & 0.1176 & 0.1263 & 0.1312 & 0.1323 & 0.1199 & 0.1222 & 0.1217 & 0.1282 & 0.132 & 0.12 \\
\hline & $1 \%$ & 0.0336 & 0.0301 & 0.0293 & 0.0307 & 0.0316 & 0.0375 & 0.0291 & 0.0302 & 0.0296 & 0.035 & 0.0332 & 0.0307 \\
\hline
\end{tabular}

\section{Analysis using the sex-specific map as employed for the simulation}

\begin{tabular}{|c|c|c|c|c|c|c|c|c|c|c|c|c|c|}
\hline \multirow[t]{4}{*}{ One marker } & $95 \%$ & 1.1858 & 1.1558 & 1.1208 & 1.1717 & 1.1595 & 1.1581 & 1.2106 & 1.1611 & 1.1381 & 1.1858 & 1.1819 & 1.1437 \\
\hline & $99 \%$ & 1.8778 & 1.7971 & 1.7768 & 1.8822 & 1.8578 & 1.815 & 1.8178 & 1.8463 & 1.7848 & 1.8704 & 1.8477 & 1.7957 \\
\hline & $5 \%$ & 0.1203 & 1104 & 1036 & 0.1112 & 0.1091 & 0.1126 & 0.1197 & 0.11 & 0.1064 & 0.1169 & 0.1151 & 0.1078 \\
\hline & $1 \%$ & 0.029 & 0.0254 & 0.0233 & 0.0268 & 0.0267 & 0.0259 & 0.0292 & 0.0255 & 0.0227 & 0.028 & 0.0254 & 0.025 \\
\hline
\end{tabular}




\begin{tabular}{|c|c|c|c|c|c|c|c|c|c|c|c|c|c|}
\hline \multirow{4}{*}{$\begin{array}{l}\text { Four } \\
\text { markers, } 1 \\
\text { cM }\end{array}$} & $95 \%$ & 1.072 & 1.0955 & 1.0979 & 1.1109 & 1.1056 & 1.104 & 1.1046 & 1.091 & 1.1037 & 1.1857 & 1.1169 & 1.1427 \\
\hline & $99 \%$ & 1.6991 & 1.8747 & 1.7806 & 1.7489 & 1.7816 & 1.816 & 1.7859 & 1.7652 & 1.8067 & 1.8625 & 1.7911 & 1.7291 \\
\hline & $5 \%$ & 0.0932 & 0.0979 & 0.0951 & 0.0972 & 0.0967 & 0.0982 & 0.0943 & 0.0942 & 0.093 & 0.1076 & 0.0979 & 0.0981 \\
\hline & $1 \%$ & 0.0188 & 0.0231 & 0.023 & 0.0227 & 0.024 & 0.0222 & 0.0236 & 0.0201 & 0.0223 & 0.272 & 0.0235 & 0.0218 \\
\hline \multirow{4}{*}{$\begin{array}{l}\text { Four } \\
\text { markers, } 10 \\
\text { cM }\end{array}$} & $95 \%$ & 1.2274 & 1.2195 & 1.1987 & 1.2344 & 1.2532 & 1.2283 & 1.2313 & 1.2519 & 1.225 & 1.2216 & 1.2248 & 1.2254 \\
\hline & $99 \%$ & 1.8604 & 1.989 & 1.8855 & 1.8541 & 1.9145 & 1.9445 & 1.9399 & 1.8588 & 1.9096 & 1.8539 & 1.8985 & 1.8966 \\
\hline & $5 \%$ & 0.1324 & 0.1244 & 0.1179 & 0.1244 & 0.1284 & 0.1313 & 0.1278 & 0.1272 & 0.1228 & 0.1299 & 0.1273 & 0.1228 \\
\hline & $1 \%$ & 0.0299 & 0.0304 & 0.0283 & 0.0301 & 0.0323 & 0.0327 & 0.0289 & 0.0311 & 0.0294 & 0.0289 & 0.0283 & 0.0319 \\
\hline \multirow[t]{4}{*}{40 SNPs } & $95 \%$ & 1.2545 & 1.2524 & 1.2274 & 1.2462 & 1.2599 & 1.2152 & 1.2196 & 1.2122 & 1.2577 & 1.2337 & 1.2415 & 1.2623 \\
\hline & $99 \%$ & 1.9474 & 1.9433 & 1.9133 & 2.0043 & 2.0074 & 1.9261 & 1.9268 & 1.9235 & 2.0406 & 1.8943 & 1.8754 & 1.9926 \\
\hline & $5 \%$ & 0.1321 & 0.1328 & 0.1246 & 0.1302 & 0.1258 & 0.1251 & 0.1229 & 0.1218 & 0.1257 & 0.1274 & 0.125 & 0.1279 \\
\hline & $1 \%$ & 0.0327 & 0.0316 & 0.0295 & 0.0333 & 0.0342 & 0.0297 & 0.0297 & 0.03 & 0.0317 & 0.0301 & 0.0308 & 0.0346 \\
\hline
\end{tabular}

Values in cM correspond to sex-averaged inter-marker distances. 95\% (99\%) quantile of a $\chi^{2}$ distribution with $1 \mathrm{df}$, divided by $2 \log (10)$ : 0.8342 (1.4407). For more details see Table 1.

\subsubsection{Twopoint analysis (1 marker)}

With regard to ASPs, the results of the analyses using a sex-averaged map showed a $H_{0, a}$ distribution of the MOBIT with smaller quantiles than the assumed $\chi_{1}^{2}$ distribution and showed no differences as to the underlying map ratios (Table 2). For the analyses using the sex-specific map and ASPs, empiric quantiles dropped for map ratios $>1$, which is due to maximization curves along the given map ratio reaching into the left half-tetrahedron $\mathbf{T}_{\mathbf{z}_{1}}^{\text {pat }}>\mathbf{z}_{1}^{\text {mat }}$ for ASPs in the nonimprinting likelihood (see also Methods Section 2.3 and Figure 2).

In the case of 3-G pedigrees (Table 3), the MOBIT distributions showed consistently larger quantiles than the assumed $\chi_{1}^{2}$ distribution, irrespective of the underlying map ratio and whether a sex-averaged or a sex-specific map was used for the analysis. Obviously, the effect of maximization curves in a sex-specific MOBIT analysis using 3-G pedigrees is restricted, possibly due to peculiarities of the parameter space around the true point in terms of allele-sharing under $H_{0, a}$.

\subsubsection{Multipoint analysis}

The $H_{0, a}$ distribution of the MOBIT for ASPs in the two four-marker scenarios and the 40-SNPs scenario when using a sex-averaged map also had lower quantiles than the assumed $\chi_{1}^{2}$ distribution and did not differ between the map ratios (Table 2). The corresponding sex-specific $H_{0, a}$ quantiles of the multipoint scenarios were not affected by maximization curves reaching into the left half-tetrahedron $\mathbf{T}_{\mathbf{z}_{1}^{\text {pat }}}>\mathbf{z}_{1}^{\text {mat }}$ for ASPs in the nonimprinting likelihood and were comparable to the quantiles of the sex-averaged analyses. This is because the maximization curves are caught between flanking markers in multipoint analyses. In addition, the maximization is multiply restricted around the true point in terms of allele-sharing under $H_{0, a}$ (see Figure 1).

For 3-G pedigrees, similar to the twopoint scenarios, the multipoint MOBIT distributions showed larger quantiles than the assumed $\chi_{1}^{2}$ distribution, irrespective of the underlying map ratio and whether a sex-averaged or a sex-specific map was used for the analysis (Table 3).

\section{$3.2 H_{o, b}$ : Linkage, no imprinting}

The results of the MOBIT analyses under the null hypothesis of linkage, but no imprinting can be found in Table 4 (ASPs) and Table 5 (3-G pedigrees).

Table 4: Empiric quantiles (95\% and 99\%) and type I error rates (nominal $\alpha=0.05 ; 0.01$ ) of the simulated ASP scenarios under $H_{0, b}$ : Linkage, no imprinting. 


\begin{tabular}{|c|c|c|c|c|c|c|c|c|c|c|c|c|}
\hline Sample size & & & 400 & & & 600 & & & 400 & & & 600 \\
\hline Map ratio & 1:1 & $7: 3$ & 9:1 & 1:1 & $7: 3$ & 9:1 & 1:1 & $7: 3$ & $9: 1$ & $1: 1$ & $7: 3$ & \\
\hline
\end{tabular}

\begin{tabular}{|c|c|c|c|c|c|c|c|c|c|c|c|c|c|}
\hline \multirow{4}{*}{$\begin{array}{l}\text { One marker, } \\
0 \mathrm{cM}\end{array}$} & $5 \%$ & 0.8127 & 0.8287 & 8586 & 0.8434 & 0.8329 & 0.8197 & 0.8369 & 0.8356 & 0.8419 & 0.836 & 0.8354 & 0.8103 \\
\hline & $99 \%$ & 1.3966 & 1.4299 & 1.487 & 1.4243 & 1.4276 & 1.4043 & 1.3917 & 1.4254 & 1.4108 & 1.4321 & 1.4884 & .3893 \\
\hline & $5 \%$ & 0.0472 & 0.0489 & 0.0539 & 0.0513 & 0.0497 & 0.048 & 0.0505 & & 0.0516 & 0506 & 505 & 0.0461 \\
\hline & $1 \%$ & 0.009 & 0.0099 & .0111 & 0.0094 & 0.0095 & 0.0084 & 0.0085 & 0.0095 & 0.0091 & 0.0099 & 0.011 & 0.0081 \\
\hline \multirow{4}{*}{$\begin{array}{l}\text { One marker, } \\
0.5 \mathrm{cM}\end{array}$} & $95 \%$ & 0.8175 & 0.8455 & 0.8625 & 0.8166 & 0.8328 & 0.8433 & 0.8273 & 0.8371 & 0.8699 & 0.8298 & 0.8595 & 0.8674 \\
\hline & $99 \%$ & 1.3664 & 1.4581 & 1.4589 & 1.4974 & 1.4654 & 1.3944 & 1.4032 & 1.4467 & 1.4678 & 1.4964 & 1.5154 & 1.4719 \\
\hline & $5 \%$ & 0.0478 & 0.0511 & 0.0539 & 0.047 & 0.0495 & 0.0511 & 0.049 & 06 & & 0.0497 & 539 & 0.0542 \\
\hline & $1 \%$ & 0.0078 & 0.0108 & 0.0111 & 0.0123 & 0.0111 & 0.0094 & 0.0092 & 0.0104 & 0.0108 & 0.0115 & 0.0118 & 0.0107 \\
\hline \multirow{4}{*}{$\begin{array}{l}\text { One marker, } \\
5 \mathrm{cM}\end{array}$} & $95 \%$ & 0.8229 & 0.8682 & 1.0221 & 0.833 & 0.886 & 1.1239 & 0.8455 & 1.3638 & 2.4917 & 0.8271 & 1.582 & 3.1716 \\
\hline & $99 \%$ & 1.4326 & 1.4871 & 1.7243 & 1.4315 & 45 & 1.8918 & & & 98 & 83 & 18 & 4.3856 \\
\hline & $5 \%$ & 0.0483 & 0.0546 & 0.079 & 0.05 & 0.0579 & 0.0896 & 18 & 0.146 & 0.4297 & 189 & 849 & 0.5909 \\
\hline & $1 \%$ & 0.01 & 0.0109 & 0.0189 & 0.0097 & 0.012 & 0.0248 & 0.0114 & 0.0431 & 0.2116 & 0.0105 & 0.0651 & 0.3456 \\
\hline \multirow{4}{*}{$\begin{array}{l}\text { Four } \\
\text { markers, } 0.5 \\
\text { cM }\end{array}$} & $95 \%$ & 0.8798 & 0.8735 & 0.8117 & 0.8393 & 0.8897 & 0.8327 & 0.8272 & 0.8543 & 0.8245 & 0.8433 & 0.8574 & 0.811 \\
\hline & $99 \%$ & 1.5429 & 1.4836 & 1.4203 & 1.5661 & 1.503 & 1.445 & 32 & 84 & 37 & & 294 & 1.405 \\
\hline & $5 \%$ & 0.0553 & 0.0558 & 0.047 & 0.0506 & 0.0577 & 0.05 & 0.0493 & 0.0531 & 0.0489 & 517 & & 0.0474 \\
\hline & $1 \%$ & 0.0123 & & 0.0097 & 0.0123 & 0.0123 & & 0.0102 & 0.01 & 0.0091 & 98 & 0.0098 & 0.0094 \\
\hline \multirow{4}{*}{$\begin{array}{l}\text { Four } \\
\text { markers, } 5 \\
\text { cM }\end{array}$} & $95 \%$ & 0.8681 & 0.899 & 0.8564 & 0.8913 & 0.9071 & 0.9268 & 0.8401 & 0.9478 & 1.2909 & 0.8519 & 1.021 & 1.4261 \\
\hline & $99 \%$ & & & & & & & & & & & & 2.2446 \\
\hline & $5 \%$ & 0.0553 & 0.0606 & 0.0541 & 0.0583 & 0.0613 & 0.0636 & 0.0504 & 0.0667 & 0.1248 & 0.0529 & 0.0754 & 0.1535 \\
\hline & $1 \%$ & 0.0111 & 0.0117 & 0.0114 & 0.0126 & 0.0135 & 0.015 & 0.0083 & 0.0155 & 0.0373 & 0.0113 & 0.0164 & 0.0494 \\
\hline \multirow[t]{4}{*}{40 SNPs } & $95 \%$ & 0.9035 & 0.9085 & 0.9077 & 0.9064 & 0.8602 & 0.8695 & 0.8694 & 0.847 & 0.8503 & 0.8431 & 0.8307 & 0.8514 \\
\hline & $99 \%$ & 1.5358 & 1.5356 & 1.5856 & 1.4931 & 1.4947 & 1.482 & 1.5095 & 1.4936 & 1.4485 & 1.4772 & 1.4455 & 1.4426 \\
\hline & $5 \%$ & & 0.0593 & 0.0582 & & & 0.0546 & & & & & & 0.0517 \\
\hline & $1 \%$ & 0.0124 & 0.0133 & 0.0133 & 0.0112 & 0.0114 & 0.011 & 0.0121 & 0.0116 & 0.0103 & 0.0112 & 0.0103 & 0.0102 \\
\hline
\end{tabular}

Analysis using the sex-specific map as employed for the simulation

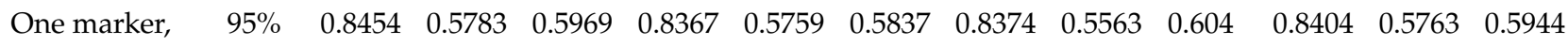
$0 \mathrm{cM}$

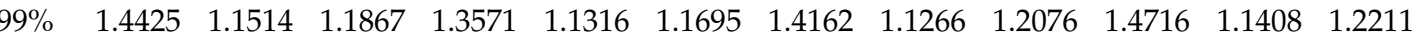

$\begin{array}{lllllllllllll}5 \% & 0.0519 & 0.0224 & 0.0268 & 0.0505 & 0.0242 & 0.0253 & 0.0508 & 0.0232 & 0.0263 & 0.0512 & 0.0249 & 0.0252\end{array}$

$\begin{array}{lllllllllllll}1 \% & 0.0102 & 0.005 & 0.0047 & 0.0086 & 0.0046 & 0.005 & 0.0096 & 0.0048 & 0.0058 & 0.0108 & 0.004 & 0.0057\end{array}$

$\begin{array}{llllllllllllll}\text { One marker, } & 95 \% & 0.8229 & 0.5614 & 0.5249 & 0.8257 & 0.5563 & 0.5409 & 0.8586 & 0.5061 & 0.4335 & 0.8357 & 0.4973 & 0.406\end{array}$

$0.5 \mathrm{cM}$

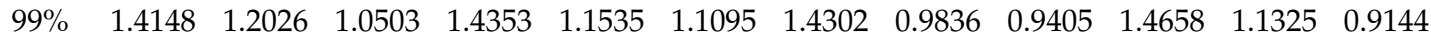

$\begin{array}{lllllllllllll}5 \% & 0.0485 & 0.0243 & 0.0191 & 0.0489 & 0.0213 & 0.0213 & 0.0541 & 0.0175 & 0.0137 & 0.0504 & 0.0187 & 0.0125\end{array}$

$\begin{array}{llllllllllllll}1 \% & 0.0094 & 0.006 & 0.0038 & 0.01 & 0.0045 & 0.0041 & 0.0099 & 0.0027 & 0.002 & 0.0109 & 0.0045 & 0.0015\end{array}$

$\begin{array}{lllllllllllllll}\text { One marker, } \quad 95 \% & 0.8351 & 0.3985 & 0.281 & 0.8166 & 0.3937 & 0.2394 & 0.8439 & 0.1885 & 0.0074 & 0.861 & 0.1061 & 0.009\end{array}$

$5 \mathrm{cM}$

$\begin{array}{lllllllllllll}99 \% & 1.4262 & 0.8971 & 0.7075 & 1.397 & 0.8739 & 0.6432 & 1.4144 & 0.5228 & 0.1073 & 1.4769 & 0.372 & 0.0218\end{array}$

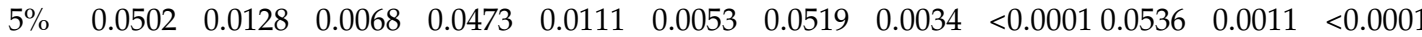

$\begin{array}{llllllllllll}1 \% & 0.0095 & 0.0025 & 0.0008 & 0.009 & 0.0013 & 0.0005 & 0.009 & 0.0004 & <0.0001 & 0.0106 & <0.0001<0.0001\end{array}$

Four $\begin{array}{lllllllllllll}95 \% & 0.8568 & 0.8711 & 0.8288 & 0.8521 & 0.8579 & 0.8194 & 0.8352 & 0.8368 & 0.8186 & 0.828 & 0.8318 & 0.8478\end{array}$

markers, 0.5

$\mathrm{cM}$

$\begin{array}{lllllllllllll}99 \% & 1.4541 & 1.5307 & 1.448 & 1.4211 & 1.4845 & 1.4173 & 1.4331 & 1.4486 & 1.3695 & 1.4455 & 1.3634 & 1.4573\end{array}$

$\begin{array}{llllllllllllll}5 \% & 0.0537 & 0.0554 & 0.0492 & 0.0527 & 0.0531 & 0.0478 & 0.0502 & 0.0507 & 0.0483 & 0.0491 & 0.0494 & 0.0522\end{array}$

$\begin{array}{lllllllllllll}1 \% & 0.0107 & 0.0125 & 0.0101 & 0.0094 & 0.0111 & 0.0095 & 0.0098 & 0.0102 & 0.0087 & 0.0101 & 0.0082 & 0.0104\end{array}$

Four $\begin{array}{lllll}95 \% & 0.8973 & 0.8649 & 0.8253 & 0.91\end{array}$ 0.88

markers, 5

$\mathrm{cM}$

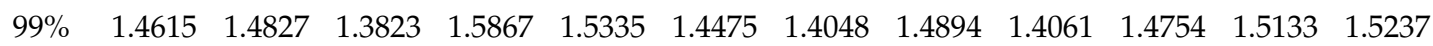

$\begin{array}{lllllllllllll}5 \% & 0.0586 & 0.0555 & 0.0486 & 0.0621 & 0.0577 & 0.052 & 0.0421 & 0.0541 & 0.0504 & 0.0498 & 0.0543 & 0.0582\end{array}$

$\begin{array}{lllllllllllll}1 \% & 0.0106 & 0.0114 & 0.0086 & 0.0136 & 0.0124 & 0.0106 & 0.0087 & 0.0112 & 0.0091 & 0.0106 & 0.0114 & 0.0127\end{array}$ 
$\begin{array}{llllllllllllll}40 \mathrm{SNPs} & 95 \% & 0.9108 & 0.8987 & 0.8362 & 0.8941 & 0.8774 & 0.8419 & 0.8582 & 0.8432 & 0.828 & 0.8312 & 0.8153 & 0.8565\end{array}$

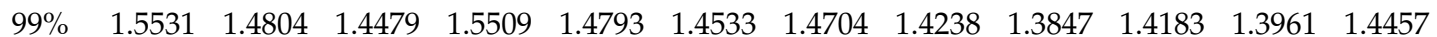
$\begin{array}{lllllllllllll}5 \% & 0.0616 & 0.0599 & 0.0502 & 0.0574 & 0.0546 & 0.0515 & 0.0529 & 0.0516 & 0.049 & 0.0497 & 0.0471 & 0.0528\end{array}$ $\begin{array}{lllllllllllll}1 \% & 0.0131 & 0.0111 & 0.0102 & 0.0138 & 0.0111 & 0.0105 & 0.0113 & 0.0097 & 0.0087 & 0.0093 & 0.009 & 0.0102\end{array}$

Values in cM correspond to sex-averaged marker-trait locus distances. $95 \%(99 \%)$ quantile of a $\chi^{2}$ distribution with $1 \mathrm{df}$, divided by $2 \log (10): 0.8342$ (1.4407). Values in bold indicate inflated quantiles and type I error rates due to confounding between sex-specific recombination fractions and genomic imprinting. For more details see Table 1.

Table 5: Empiric quantiles (95\% and 99\%) and type I error rates (nominal $\alpha=0.05 ; 0.01$ ) of the simulated 3-G pedigree scenarios under $H_{0, b}$ : Linkage, no imprinting.

\begin{tabular}{|c|c|c|c|c|c|c|c|c|c|c|c|c|c|}
\hline & & & & & & & & \multicolumn{6}{|c|}{ 3-G pedigrees } \\
\hline Trait model & & \multicolumn{6}{|c|}{ additive } & \multicolumn{6}{|c|}{ recessive } \\
\hline Sample size & & \multicolumn{3}{|r|}{45} & \multicolumn{3}{|r|}{65} & \multicolumn{3}{|r|}{45} & \multicolumn{3}{|r|}{65} \\
\hline Map ratio & & 1:1 & $7: 3$ & 9:1 & 1:1 & $7: 3$ & 9:1 & 1:1 & $7: 3$ & $9: 1$ & 1:1 & $7: 3$ & 9:1 \\
\hline \multicolumn{14}{|c|}{ Analysis using a sex-averaged map } \\
\hline \multirow{4}{*}{$\begin{array}{l}\text { One marker, } \\
0 \mathrm{cM}\end{array}$} & $95 \%$ & 1.0483 & 1.0374 & 1.0672 & 0.9659 & 1.0291 & 0.9771 & 0.8145 & 0.8082 & 0.821 & 0.8021 & 0.8145 & 0.8275 \\
\hline & $99 \%$ & 1.8388 & 1.7556 & 1.7557 & 1.6499 & 1.7091 & 1.6911 & 1.3563 & 1.3998 & 1.4 & 1.3584 & 1.4378 & 1.354 \\
\hline & $5 \%$ & 0.0791 & 0.0802 & 0.0871 & 0.0696 & 0.0777 & 0.0741 & 0.0474 & 0.0467 & 0.0476 & 0.0447 & 0.0477 & 0.0493 \\
\hline & $1 \%$ & 0.0214 & 0.0201 & 0.0199 & 0.018 & 0.0176 & 0.0172 & 0.0076 & 0.0087 & 0.0085 & 0.0081 & 0.0099 & 0.0084 \\
\hline \multirow{4}{*}{$\begin{array}{l}\text { One marker, } \\
0.5 \mathrm{cM}\end{array}$} & $95 \%$ & 1.0655 & 1.0573 & 1.055 & 0.9983 & 0.9878 & 1.0228 & 0.8253 & 0.8433 & 0.8362 & 0.8157 & 0.8685 & 0.827 \\
\hline & $99 \%$ & 1.7972 & 1.6659 & 1.7346 & 1.6928 & 1.7089 & 1.7136 & 1.3968 & 1.4412 & 1.4075 & 1.4293 & 1.4628 & 1.4281 \\
\hline & $5 \%$ & 0.0874 & 0.0855 & 0.0813 & 0.075 & 0.0726 & 0.0759 & 0.0493 & 0.0519 & 0.0506 & 0.0485 & 0.0549 & 0.0489 \\
\hline & $1 \%$ & 0.0231 & 0.0189 & 0.0208 & 0.0185 & 0.019 & 0.0194 & 0.009 & 0.101 & 0.0095 & 0.0099 & 0.0107 & 0.0096 \\
\hline \multirow{4}{*}{$\begin{array}{l}\text { One marker, } \\
5 \mathrm{cM}\end{array}$} & $95 \%$ & 1.0941 & 1.1011 & 1.0998 & 1.0668 & 1.0603 & 1.1036 & 0.8632 & 0.9687 & 1.2511 & 0.9032 & 1.026 & 1.4039 \\
\hline & $99 \%$ & 7775 & 77 & 1.75 & 211 & 1.8 & 1.7925 & 1.4 & 94 & 2.075 & 1.5279 & 63 & 2.2133 \\
\hline & $5 \%$ & 0.0909 & 0.0916 & 0.0953 & 0.0911 & 0.0855 & 0.0916 & 0.0535 & & 0.1115 & 0.0607 & 0.0778 & 0.144 \\
\hline & $1 \%$ & 0.0222 & 0.0248 & 0.0235 & 0.0222 & 0.0221 & 0.0233 & 0.0104 & 0.0164 & 0.0347 & 0.0129 & 0.0194 & 0.0468 \\
\hline \multirow{4}{*}{$\begin{array}{l}\text { Four } \\
\text { markers, } 0.5 \\
\text { cM }\end{array}$} & $95 \%$ & 0.9891 & 1.0036 & 0.9493 & 0.9584 & 0.9382 & 0.9408 & 0.8354 & 0.808 & 0.7827 & 0.8024 & 0.8139 & 0.775 \\
\hline & $99 \%$ & 1.7004 & 1.6667 & 1.6085 & 1.6035 & 1.6 & 1.6464 & & & 1.3825 & 1.3559 & & 1.3931 \\
\hline & $5 \%$ & 0.0741 & 0.0755 & 0.0653 & 0.0668 & 0.0648 & 0.0646 & 0.0501 & 0.0462 & 0.0439 & 0.0462 & 0.0476 & 0.0419 \\
\hline & $1 \%$ & 0.0167 & 0.0167 & 0.0142 & 0.0147 & 0.0154 & 0.0161 & 0.0101 & 0.0092 & 0.0083 & 0.0083 & 0.0115 & 0.0089 \\
\hline \multirow{4}{*}{$\begin{array}{l}\text { Four } \\
\text { markers, } 5 \\
\text { cM }\end{array}$} & $95 \%$ & 1.0746 & 1.0712 & 1.0277 & 1.0109 & 1.027 & 1.0074 & 0.7978 & 0.8499 & 0.95 & 0.806 & 0.8633 & 0.9306 \\
\hline & $99 \%$ & 1.7492 & 1.7618 & 1.7313 & 1.6572 & 1.6982 & 1.6708 & 1.4304 & 1.4569 & 1.5372 & 1.3541 & 1.4902 & 1.6792 \\
\hline & $5 \%$ & 0.0848 & 0.0887 & 0.0788 & 0.0758 & 0.0781 & 0.0777 & 0.0461 & 0.052 & 0.0653 & 0.0465 & 0.0541 & 0.0638 \\
\hline & $1 \%$ & 0.0209 & 0.023 & 0.0182 & 0.018 & 0.0195 & 0.0169 & 0.0096 & 0.0106 & 0.0138 & 0.009 & 0.0116 & 0.0161 \\
\hline \multirow[t]{4}{*}{40 SNPs } & $95 \%$ & 1.0386 & 1.0255 & 1.0074 & 0.9789 & 0.9925 & 0.9815 & 0.7949 & 0.8175 & 0.817 & 0.8381 & 0.8209 & 0.8072 \\
\hline & $99 \%$ & 1.7208 & 1.726 & 1.7 & 1.58 & 1.619 & 1.7236 & 1.4125 & 1.4452 & 1.4265 & 1.4346 & 1.4263 & 1.4496 \\
\hline & $5 \%$ & 0.0819 & 0.077 & 0.0741 & 0.0726 & 0.0715 & 0.0709 & 0.045 & 0.0477 & 0.047 & 0.0507 & 0.0482 & 0.0471 \\
\hline & $1 \%$ & 0.0191 & 0.0191 & 0.0184 & 0.0147 & 0.0155 & 0.0177 & 0.0092 & 0.0102 & 0.0097 & 0.0099 & 0.0095 & 0.0103 \\
\hline
\end{tabular}

Analysis using the sex-specific map as employed for the simulation

\begin{tabular}{|c|c|c|c|c|c|c|c|c|c|c|c|c|c|}
\hline \multirow{4}{*}{$\begin{array}{l}\text { One marker, } \\
0 \mathrm{cM}\end{array}$} & $95 \%$ & 1.0608 & 0.9992 & 0.9616 & 0.9594 & 0.9349 & 0.9317 & 0.8177 & 0.7607 & 0.7583 & 0.8074 & 0.797 & 0.7729 \\
\hline & $99 \%$ & 1.7583 & 1.6683 & 1.5927 & 1.6848 & 1.5877 & 1.6011 & 1.3415 & 1.3163 & 1.3395 & 1.3737 & 1.3355 & 1.3541 \\
\hline & $5 \%$ & 0.0792 & 0.0757 & 0.0687 & 0.0701 & 0.0644 & 0.0658 & 0.0482 & 0.0406 & 0.0391 & 0.0461 & 0.0447 & 0.0393 \\
\hline & $1 \%$ & 0.0192 & 0.0174 & 0.0141 & 0.0173 & 0.0136 & 0.0135 & 0.0072 & 0.0069 & 0.0076 & 0.0083 & 0.0071 & 0.0084 \\
\hline \multirow{4}{*}{$\begin{array}{l}\text { One marker, } \\
0.5 \mathrm{cM}\end{array}$} & $95 \%$ & 1.0663 & 0.9906 & 0.9635 & 0.9749 & 0.9458 & 0.9498 & 0.8186 & 0.7503 & 0.716 & 0.8314 & 0.7356 & 0.7102 \\
\hline & $99 \%$ & 1.7535 & 1.6446 & 1.6307 & 1.6397 & 1.5692 & 1.6368 & 1.3805 & 1.2754 & 1.2609 & 1.3691 & 1.2924 & 1.3014 \\
\hline & $5 \%$ & 0.0817 & 0.0723 & 0.0711 & 0.0712 & 0.0667 & 0.0663 & 0.0473 & 0.0378 & 0.0359 & 0.0496 & 0.0364 & 0.0358 \\
\hline & $1 \%$ & 0.0214 & 0.0171 & 0.0156 & 0.0176 & 0.0149 & 0.0154 & 0.0089 & 0.0067 & 0.0055 & 0.0082 & 0.0063 & 0.007 \\
\hline $\begin{array}{l}\text { One marker, } \\
5 \mathrm{cM}\end{array}$ & $95 \%$ & 1.1129 & 0.9857 & 0.974 & 1.0475 & 0.9476 & 0.9565 & 0.8493 & 0.719 & 0.658 & 0.826 & 0.7171 & 0.6731 \\
\hline
\end{tabular}




\begin{tabular}{|c|c|c|c|c|c|c|c|c|c|c|c|c|c|}
\hline & $99 \%$ & 1.7828 & 1.6597 & 1.6038 & 1.741 & 1.6496 & 1.6168 & 1.4627 & 1.2505 & 1.167 & 1.4412 & 1.17 & 1.1188 \\
\hline & $5 \%$ & 0.0941 & 0.0732 & 0.0731 & 0.0855 & 0.0679 & 0.0689 & 0.0526 & 0.035 & 0.0288 & 0.0487 & 0.0325 & 0.0299 \\
\hline & $1 \%$ & 0.0232 & 0.0173 & 0.0158 & 0.0189 & 0.017 & 0.0157 & 0.0106 & 0.0065 & 0.0036 & 0.0101 & 0.0055 & 0.0029 \\
\hline \multirow{4}{*}{$\begin{array}{l}\text { Four } \\
\text { markers, } 0.5 \\
\text { cM }\end{array}$} & $95 \%$ & 0.9874 & 1.0233 & 0.9818 & 0.9555 & 0.9942 & 0.938 & 0.8088 & 0.8155 & 0.8084 & 0.7908 & 0.7968 & 0.8094 \\
\hline & $99 \%$ & 1.7174 & 1.7472 & 1.6581 & 1.6428 & 1.7033 & 1.6004 & 1.3616 & 1.3805 & 1.4248 & 1.3701 & 1.3075 & 1.4083 \\
\hline & $5 \%$ & 0.0723 & 0.0782 & 0.0723 & 0.0682 & 0.0717 & 0.0651 & 0.0474 & 0.0469 & 0.0466 & 0.0436 & 0.0456 & 0.0463 \\
\hline & $1 \%$ & 0.0172 & 0.0186 & 0.0162 & 0.0161 & 0.0184 & 0.0144 & 0.0077 & 0.0086 & 0.0096 & 0.0086 & 0.0075 & 0.0095 \\
\hline \multirow{4}{*}{$\begin{array}{l}\text { Four } \\
\text { markers, } 5 \\
\text { cM }\end{array}$} & $95 \%$ & 1.0651 & 1.0691 & 1.0344 & 1.0168 & 1.0063 & 0.9618 & 0.8206 & 0.825 & 0.7817 & 0.8199 & 0.822 & 0.7875 \\
\hline & $99 \%$ & 1.7923 & 1.7826 & 1.7352 & 1.7108 & 1.61 & 1.6321 & 1.4158 & 1.4263 & 1.4319 & 1.4207 & 1.427 & 1.3247 \\
\hline & $5 \%$ & 0.0872 & 0.0878 & 0.0804 & 0.0764 & 0.0755 & 0.0682 & 0.0486 & 0.0482 & 0.0435 & 0.0484 & 0.0481 & 0.0424 \\
\hline & $1 \%$ & 0.0221 & 0.0205 & 0.0193 & 0.0188 & 0.016 & 0.0168 & 0.0094 & 0.0097 & 0.0099 & 0.0098 & 0.0098 & 0.0072 \\
\hline \multirow[t]{4}{*}{40 SNPs } & $95 \%$ & 1.0363 & 0.9697 & 1.0075 & 0.9758 & 1.0128 & 0.9701 & 0.8032 & 0.8068 & 0.8105 & 0.8163 & 0.8373 & 0.8135 \\
\hline & $99 \%$ & 1.6783 & 1.6172 & 1.6526 & 1.665 & 1.6832 & 1.563 & 1.3855 & 1.3863 & 1.4469 & 1.3599 & 1.4731 & 1.4228 \\
\hline & $5 \%$ & 0.0831 & 0.0718 & 0.0746 & 0.0699 & 0.0765 & 0.0676 & 0.0468 & 0.0466 & 0.0471 & 0.0473 & 0.0508 & 0.0473 \\
\hline & $1 \%$ & 0.0185 & 0.0162 & 0.0176 & 0.0162 & 0.0184 & 0.0141 & 0.0086 & 0.0091 & 0.0102 & 0.008 & 0.011 & 0.0089 \\
\hline
\end{tabular}

Values in cM correspond to sex-averaged marker-trait locus distances. $95 \%(99 \%)$ quantile of a $\chi^{2}$ distribution with $1 \mathrm{df}$, divided by $2 \log (10): 0.8342$ (1.4407). Values in bold indicate inflated quantiles and type I error rates due to confounding between sex-specific recombination fractions and genomic imprinting. For more details see Table 1.

\subsubsection{Twopoint, sex-averaged analysis}

With respect to ASPs and the additive as well as the recessive trait model, the $H_{0, b}$ distribution of the MOBIT for the scenario with $0 \mathrm{cM}$ between marker and trait locus corresponded well to the expected $\chi^{2}$ distribution with $1 \mathrm{df}$ for all map ratios and using a sex-averaged map in the analysis (Table 4). Although the additive trait model lies on the boundary of the tetrahedron $\mathbf{T}$ in terms of allele-sharing for ASPs, it is readily conceivable that the two front planes in Figure 1 (left side) are explored in two dimensions with the IMOD score and in one dimension with the MOD score, corresponding to a difference in maximized parameters of $2-1=1$, which is the same as for the interior of $\mathbf{T}(3-2=1)$. Taken together, this results in empiric MOBIT quantiles equal to those of a $\chi_{1}^{2}$ distribution. The corresponding quantiles for the scenario with $0.5 \mathrm{cM}$ between marker and trait locus for both trait models corresponded to the expected $\chi_{1}^{2}$ distribution for the 1:1 map ratio, with some inflation when moving to the 7:3 and further to the 9:1 map ratio due to confounding. In the case of the scenario with $5 \mathrm{cM}$ between marker and trait locus, MOBIT quantiles were slightly inflated for the 7:3 map ratio and clearly inflated for the 9:1 map ratio due to confounding (Table 4). Confounding was more severe for the larger sample size, the recessive trait model, and the larger marker-trait locus distance.

In the case of 3-G pedigrees and the additive trait model, the MOBIT $H_{0, b}$ quantiles of the twopoint scenarios were clearly inflated compared to the expected $\chi^{2}$ distribution with $1 \mathrm{df}$ (Table 5). This is probably because the true point in terms of allele-sharing of the additive model also lies on the boundary of the parameter space of 3-G pedigrees as it is for ASPs. In contrast to ASPs, however, this apparently does not lead to $1 \mathrm{df}$ for the MOBIT, presumably because the parameter space of 3-G pedigrees has a more complicated form with more boundaries. Further, the inflation increased with increasing marker-trait locus distance, however, additional inflation of quantiles due to confounding could not be observed (Table 5). With regard to the recessive model, the $H_{0, b}$ distribution of the MOBIT for the scenarios with 0 and $0.5 \mathrm{cM}$ between marker and trait locus corresponded well to the expected $\chi^{2}$ distribution with $1 \mathrm{df}$ for all map ratios and using a sex-averaged map in the analysis (Table 5). For the scenario with $5 \mathrm{cM}$ between marker and trait locus, however, inflation of MOBIT quantiles and hence increased type I error rates due to confounding could be observed.

As can be seen from Table 4 and Table 5, confounding of twopoint scenarios was more severe for ASPs than for 3-G pedigrees, especially for the recessive trait model (observed type I error rates for the $5 \mathrm{cM}$ twopoint scenario and a 9:1 map ratio assuming a $\chi_{1}^{2}$ distribution with a nominal $\alpha=5 \%$ significance level: 0.5909 and 0.144 for 600 ASPs and $653-G$ pedigrees, respectively).

\subsubsection{Twopoint, sex-specific analysis}

With regard to ASPs and the scenario with $0 \mathrm{cM}$ between marker and trait locus, MOBIT quantiles were significantly lower than those for the expected $\chi_{1}^{2}$ distribution due to maximization curves reaching into the left 
half-tetrahedron $\mathbf{T}_{\mathbf{z}_{1}^{\text {pat }}}>\mathbf{z}_{1}^{\text {mat }}$ for ASPs in the nonimprinting likelihood for map ratios $>1$ (Table 4). Quantiles were even lower for the scenario with $0.5 \mathrm{cM}$ between marker and trait locus and map ratios $>1$, especially for the recessive trait model. The results for the $0.5 \mathrm{cM}$ scenario were comparable between the two sample sizes. In the case of the scenario with $5 \mathrm{cM}$ between marker and trait locus, the deflation of quantiles was increasingly severe for the larger map ratio, the larger sample size, and the recessive trait model.

With 3-G pedigrees, MOBIT quantiles were deflated with an increasing map ratio and an increasing markertrait locus distance for both trait models (Table 5). The degree to which maximization curves according to a given sex-specific map ratio deflate the MOBIT was smaller for 3-G pedigrees compared to ASPs. This might be due to the complexity of the parameter space for 3-G pedigrees, such that more spatial restrictions prevent the maximization curves from reaching farther into the interior of the parameter space.

\subsubsection{Multipoint, sex-averaged analysis}

In the case of the 4 markers, $0.5 \mathrm{cM}$ scenario, the $H_{0, b}$ distribution of the MOBIT for all map ratios and both trait models corresponded to the expected $\chi^{2}$ distribution with $1 \mathrm{df}$ for ASPs (Table 4). As explained above, slightly higher quantiles for multipoint analysis are due to the increased effective number of tests compared to twopoint analyses. With respect to the 4 markers, $5 \mathrm{cM}$ scenario and the additive trait model, quantiles were slightly inflated due to confounding for the larger sample size and a map ratio of 9:1. In the case of the recessive trait model, quantiles were increasingly inflated due to confounding for larger map ratios and sample sizes. Again, confounding was more severe for the recessive trait model. The scenario with 40 SNPs and a marker spacing of $0.32 \mathrm{cM}$ did not show confounding.

In summary, a marker spacing of $1 \mathrm{cM}$ (corresponding to a marker-trait locus distance of $0.5 \mathrm{cM}$ to both flanking markers) seemed to be sufficient to avoid confounding, even in the case of such an extreme map ratio as 9:1.

In the case of 3-G pedigrees and the additive trait model, MOBIT quantiles were higher than compared to the expected $\chi^{2}$ distribution with $1 \mathrm{df}$ for all multipoint scenarios (Table 5) due to the above mentioned boundary conditions of the true point in terms of allele sharing in the parameter space of 3-G pedigrees. Slightly higher MOBIT quantiles were obtained for the 4 markers, $5 \mathrm{cM}$ scenario compared to the 4 markers, $0.5 \mathrm{cM}$ scenario. The quantiles of the 40 SNPs scenario mostly lay between the two other multipoint scenarios. Despite the different quantiles for the three marker settings, there was no evidence for confounding for all multipoint scenarios and the additive trait model. The corresponding quantiles for the recessive trait model corresponded to the expected $\chi^{2}$ distribution with $1 \mathrm{df}$ for all map ratios, except for the 4 markers, $5 \mathrm{cM}$ scenario and a map ratio of 9:1 due to confounding (Table 5).

Again, confounding was more severe for ASPs than for 3-G pedigrees, especially for the recessive trait model (type I error rates for the 4 markers, $5 \mathrm{cM}$ scenario and a 9:1 map ratio assuming a $\chi_{1}^{2}$ distribution with a nominal $\alpha=5 \%$ significance level: 0.1535 and 0.0638 for 600 ASPs and $653-G$ pedigrees, respectively).

\subsubsection{Multipoint, sex-specific analysis}

Due to the multipoint setting, in which the putative trait locus is confined between flanking markers, such that the outreach of maximization curves is limited, all investigated multipoint distributions roughly corresponded to the expected $\chi^{2}$ distribution with $1 \mathrm{df}$ for ASPs (Table 4). There was only a slight deflation of quantiles for the multipoint scenarios with a 9:1 map ratio and the additive trait model. The results did not substantially differ between the two sample sizes.

In the case of the 3-G pedigrees and the additive trait model, MOBIT quantiles were higher than expected as was explained above. Quantiles only slightly decreased with increasing map ratio (Table 5). In the case of the recessive model, the distributions roughly corresponded to the expected $\chi^{2}$ distribution with $1 \mathrm{df}$. Apart from the 4 markers, $5 \mathrm{cM}$ scenario, MOBIT quantiles were not deflated due to a map ratio $>1$.

\section{3 $\quad H_{1}$ : Linkage, imprinting}

The results of the power calculations for the twopoint scenarios and the 40 SNPs, $0.32 \mathrm{cM}$ scenario can be found in Figure 4 and Figure 5. The critical values for a test with a true type I error rate of $5 \%$ corresponded to the respective $H_{0, b} 95 \%$ quantiles of each particular scenario (Table 4 and Table 5 for ASPs and 3-G pedigrees, respectively). In general, power was higher for the larger sample size for both pedigrees. 

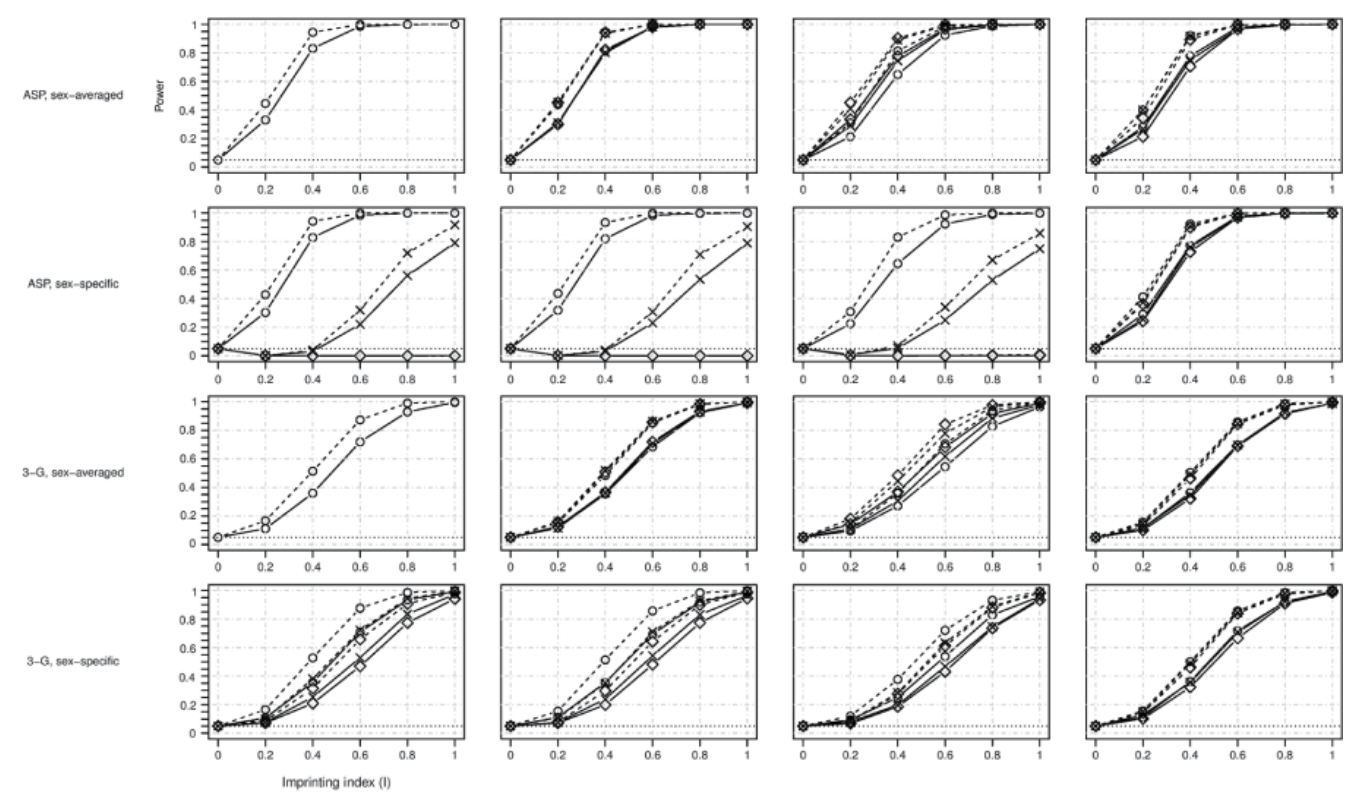

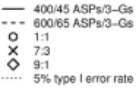

$1 \mathrm{M} ., 0 \mathrm{cM}$

$1 \mathrm{M} ., 0.5 \mathrm{cM}$

$1 \mathrm{M} ., 5 \mathrm{cM}$

40 SNPs, $0.32 \mathrm{cM}$

Figure 4: Power to detect imprinting using the MOBIT. For more details see Figure 3.
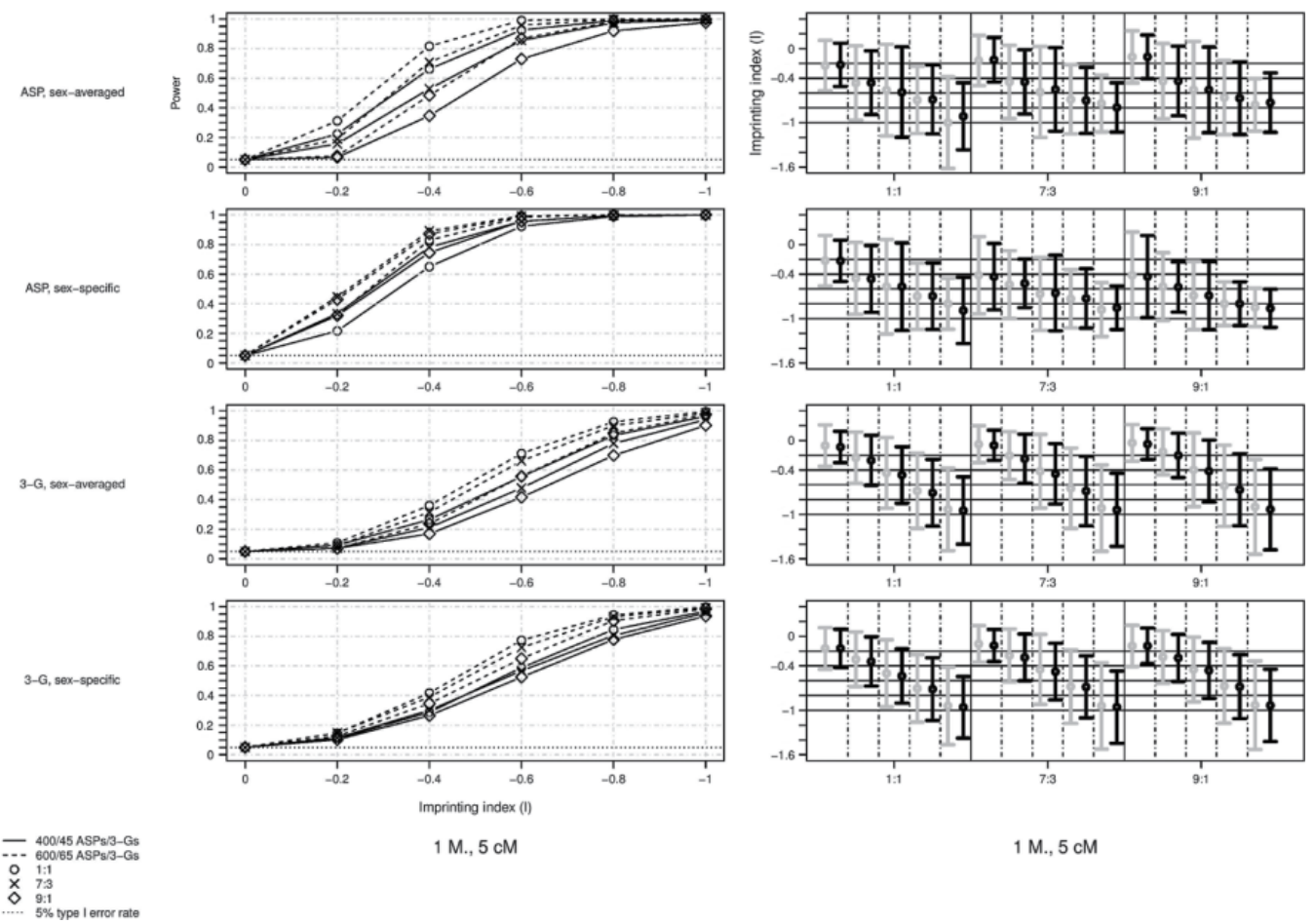

$1 \mathrm{M} ., 5 \mathrm{cM}$

$1 \mathrm{M} ., 5 \mathrm{cM}$

Figure 5: Power and estimation accuracy of imprinting index I of the MOBIT for the paternal imprinting model. Median estimates of $I$ are depicted as bullets, the corresponding median absolute deviation (MAD; adjusted by a constant (1.4826) for asymptotically normal consistency) is shown as error bars, and the larger sample size is colored in black. The scenarios are sorted in increasing order based on the absolute value of $I$ from left to right. For more details see Figure 3.

\subsubsection{Twopoint, sex-averaged analysis, maternal imprinting}

The power results for the maternal imprinting model using a sex-averaged map in the analysis can be found in Figure 4 (columns 1-3, rows 1 and 3 for ASPs and 3-G pedigrees, respectively). For the 1 marker, 0 cM scenario, results for map ratios larger than 1 are not shown, because if $\theta=0$ between marker and trait locus, the existence of sex-specific recombination fractions in the marker-surrounding genetic region is of no relevance when using a sex-averaged map for the analysis (see also Table 4 and Table 5 for the respective type I error rates under $H_{0, b}$ ). 
The power of the MOBIT was not affected by the underlying map ratio in the case of the $0.5 \mathrm{cM}$ scenario across both pedigree types. However, for the $5 \mathrm{cM}$ scenario, larger map ratios showed slightly higher power than smaller ones across both pedigree types. In general, power was higher for ASPs compared to 3-G pedigrees across all investigated twopoint scenarios and was slightly lower for larger marker-trait locus distances. In the case of the $0 \mathrm{cM}$ and $0.5 \mathrm{cM}$ scenarios, a power consistently $>80 \%$ was obtained for an imprinting index $I=0.4$ with ASPs for both sample sizes, whereas power was consistently $>80 \%$ for $I=0.8$ with $3-G$ pedigrees for both sample sizes. The corresponding values to obtain a power consistently $>80 \%$ for the $5 \mathrm{cM}$ scenario were $I=0.6$ with ASPs for both sample sizes and $I=0.8$ with 3-G pedigrees for both sample sizes.

\subsubsection{Twopoint, sex-averaged analysis, paternal imprinting}

The results of the power analysis for the paternal imprinting model and a marker-trait locus distance of $5 \mathrm{cM}$ can be found in Figure 5 (left). For small to moderate imprinting degrees and a map ratio larger than 1, power was lower compared to the corresponding maternal imprinting scenarios in Figure 4 for both pedigree types. This is due to the fact that, in the case of ASPs, the true point in terms of allele-sharing is gradually shifted from $\mathbf{T}_{\mathbf{z}_{1}^{\text {pat }}>\mathbf{z}_{1}^{\text {mat }}}$ into $\mathbf{T}_{\mathbf{z}_{1}^{\text {pat }}<\mathbf{z}_{1}^{\text {mat }}}$ with increasing imprinting degrees, thereby crossing the possible triangle. In the case of the 1:1 map ratio, however, the corresponding points in terms of allele-sharing instantly lie within $\mathbf{T}_{\mathbf{z}_{1}^{\text {pat }}}<\mathbf{z}_{1}^{\text {mat }}$ even for small imprinting degress and can exclusively be reached by the IMOD score maximization. A similar behaviour was observed for 3-G pedigrees.

\subsubsection{Multipoint, sex-averaged analysis, maternal imprinting}

The power results of the MOBIT for the multipoint scenarios differed only slightly between the map ratios and were generally higher for larger sample sizes (Figure 4, column 4, rows 1 and 3 for ASPs and 3-G pedigrees, respectively). Power was higher for ASPs compared to 3-G pedigrees. More specifically, a power consistently $>80 \%$ was obtained for an imprinting index $I=0.6$ with ASPs for both sample sizes, whereas power was consistently $>80 \%$ for $I=0.8$ with $3-G$ pedigrees for both sample sizes.

\subsubsection{Twopoint, sex-specific analysis, maternal imprinting}

Regarding the results of the MOBIT for ASPs and the maternal imprinting model, the scenarios with a map ratio of 1:1 had the highest power, with higher values for larger sample sizes and higher imprinting degrees, followed by the scenarios with a map ratio of 7:3 (Figure 4, columns 1-3, row 2). The twopoint scenarios with a map ratio of 9:1 had no power to detect imprinting, which was due to the problem of maximization curves (Figure 2). The power even dropped below the nominal type I error rate of $5 \%$. This can be explained by the fact that under $H_{0, b}$, where $I=0$, a MOBIT greater zero results mainly from maxima in terms of allele sharing due to sampling variation of the simulated replicates in the right half-tetrahedron $\mathbf{T}_{\mathbf{z}_{1}^{\text {pat }}}<\mathbf{z}_{1}^{\text {mat }}$ (for ASPs), which is exclusively explored by the imprinting (IMOD score) analysis. An imprinting effect $I>0$ shifts the point into the left half-tetrahedron $\mathbf{T}_{\mathbf{z}_{1}^{\text {pat }}}>\mathbf{z}_{1}^{\text {mat }}$, leading to maxima in the right half-tetrahedron being less likely. Due to the maximization curves, with an assumed map ratio of 9:1, the sample maxima are covered by the nonimprinting (MOD score) maximization as well as by the imprinting maximization, resulting in a power below $5 \%$. In the case of the 7:3 map ratio, the same effect is appreciable for $I=0.2$, whereas for $I \geq 0.4$, the stronger imprinting outweighs this effect of maximization curves. In the case of ASPs, there was almost no difference in power between the scenarios with varying marker-trait locus distances $(0 \mathrm{cM}, 0.5 \mathrm{cM}$, and $5 \mathrm{cM})$ for map ratios $>1$.

With 3-G pedigrees, the problem of maximization curves seemed to be smaller. The scenarios with a 1:1 map ratio and a larger sample size still had the highest power (Figure 4, columns 1-3, row 4). Interestingly, the other two map ratios showed comparable power, with again slightly higher power for scenarios with a map ratio of 7:3 compared to 9:1. In the case of 3-G pedigrees, power was slightly lower for larger marker-trait locus distances. In addition, power was consistently higher for 3-G pedigrees compared to ASP analyses in the case of a map ratio $>1$. 


\subsubsection{Twopoint, sex-specific analysis, paternal imprinting}

The power for the paternal imprinting model when using a sex-specific map in the analysis (see Figure 5, left) was substantially higher compared to the respective maternal imprinting model for both pedigree types as depicted in Figure 4. This is because maximization curves of the nonimprinting MOD score are restricted to points in terms of allele-sharing that correspond to excess paternal sharing, such that points corresponding to excess maternal sharing are exclusively covered by the IMOD score maximization. Further, the power was higher with increasing map ratio for ASPs due to smaller empiric threshold values for the MOBIT as derived from the respective $H_{0, b}$ simulations (see Table 4). The power was also higher compared to the corresponding sex-averaged analyses, for which empiric threshold values for the MOBIT under $H_{0, b}$ were inflated due to confounding (see Table 4). Conversely, the power of the 3-G pedigress was comparable to that of the respective sex-averaged analyses and was slightly higher for smaller map ratios, similar to the findings in Results Section 3.3.4.

\subsubsection{Multipoint, sex-specific analysis, maternal imprinting}

The power results of the MOBIT for the multipoint scenarios depended only slightly on the map ratio, because the putative disease locus is confined between flanking markers, which largely avoids maximization curves. Power values for the 1:1 map ratio were somewhere between the 1 marker, $0.5 \mathrm{cM}$ and 1 marker, $5 \mathrm{cM}$ scenarios with higher power observed for larger sample sizes and ASPs (Figure 4, column 4, rows 2 and 4).

\subsection{Estimation of imprinting index I in a sex-averaged MOBIT analysis}

\subsubsection{Twopoint analysis, maternal imprinting}

The twopoint imprinting parameter estimation results of the sex-averaged MOBIT analyses for ASPs can be found in Figure 6 (rows 1-3). The estimated median imprinting indices were close to their expected values for the 0 and $0.5 \mathrm{cM}$ scenarios. In the case of the $5 \mathrm{cM}$ scenario, imprinting indices $<0.6$ were mostly overestimated, whereas imprinting indices $>0.6$ were mostly underestimated. For the larger sample size, the underestimation was less pronounced. For a given map ratio, the variation as measured by the median absolute deviation $(M A D)$ was highest for $I=0.6$ and lowest for $I=0.2$. Further, $M A D$ was slightly lower for the larger sample size for most investigated scenarios.

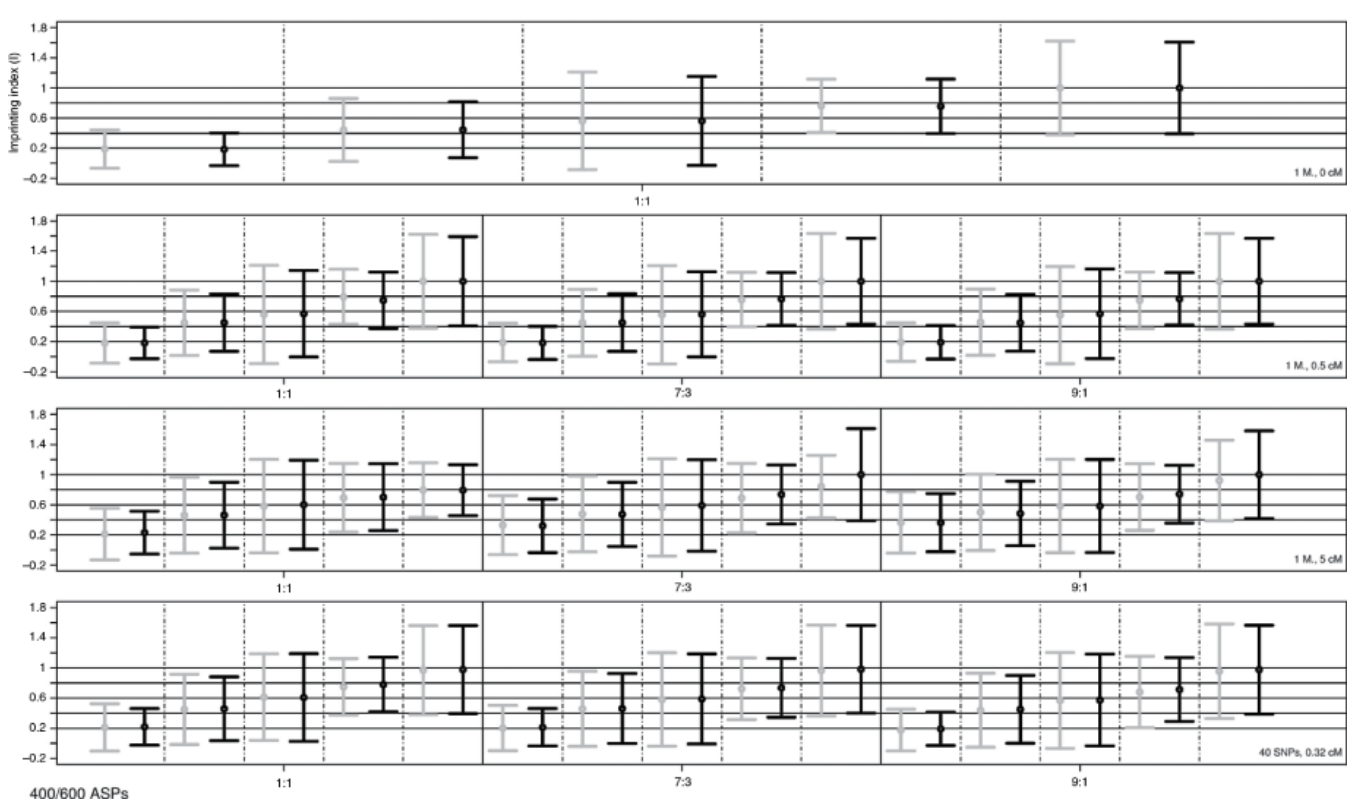

Figure 6: Estimation of imprinting index I by the MOBIT using a sex-averaged map in the analysis of ASPs. For more details see Figure 3 and Figure 5.

The corresponding imprinting parameter estimation results for 3-G pedigrees can be found in Figure 7 (rows 1-3). Median values of the estimated imprinting indices were close to their expected values, although 
underestimated, especially in the case of lower imprinting indices. $M A D$ was lowest for $I=0.2$ and slightly increased with larger imprinting indices and larger marker-trait locus distances, but did not substantially differ between map ratios of a given marker-trait locus distance. $M A D$ was lower for the larger sample size for all investigated scenarios. For most scenarios, $M A D$ of the imprinting index was lower for 3-G pedigrees compared to ASPs.
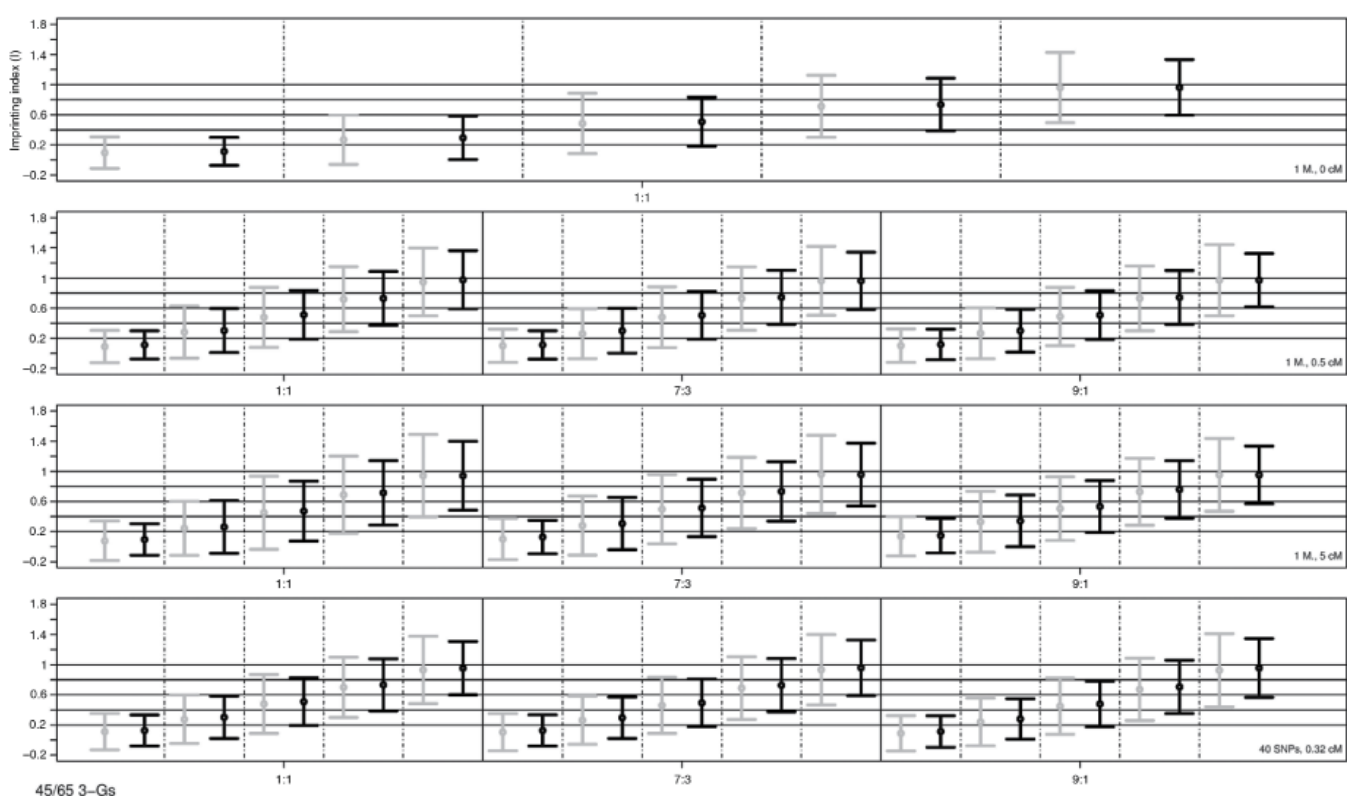

Figure 7: Estimation of imprinting index $I$ by the MOBIT using a sex-averaged map in the analysis of 3-G pedigrees. For more details see Figure 3 and Figure 5.

\subsubsection{Twopoint analysis, paternal imprinting}

The parameter estimation results for the paternal imprinting model using a sex-averaged map in the analysis can be found in Figure 5 (right, rows 1 and 3). The results for both pedigree types and the 1:1 map ratio were comparable to those of the respective maternal imprinting scenario (see Figure 6 and Figure 7, row 3). Specifically, the point estimate of the imprinting index was either close to or slightly less negative than its expected value. In the case of a map ratio larger than 1, estimates were often less negative than the expected values. This is because excess maternal allele-sharing at the disease locus is attenuated by a longer female genetic map, which reduces the maternal sharing excess at the marker locus.

\subsubsection{Multipoint analysis}

The multipoint results of the imprinting parameter estimation for the sex-averaged MOBIT analyses using ASPs can be found in Figure 6 (row 4). Estimated median imprinting indices were close to their expected values across all map ratios. $M A D$ was highest for $I=0.6$ and lowest for $I=0.2$. MAD was lower for the larger sample size, for most investigated scenarios, and did not substantially differ between map ratios.

The corresponding results for 3-G pedigrees are shown in Figure 7 (row 4). Imprinting indices were slightly underestimated across all map ratios. The underestimation was even a bit stronger than for the twopoint scenarios in the case of the 7:3 and 9:1 map ratios. $M A D$ was lowest for $I=0.2$ and slightly increased with larger imprinting indices. Again, $M A D$ was lower for the larger sample size and was comparable with respect to different map ratios. In addition, MAD of the imprinting index was lower for 3-G pedigrees compared to ASPs.

\subsection{Estimation of imprinting index I in a sex-specific MOBIT analysis}

\subsubsection{Twopoint analysis, maternal imprinting}

The twopoint results of the estimation of the imprinting index I using a sex-specific map in a MOBIT analysis using ASPs can be found in Figure 8 (rows 1-3). Apart from differences due to sampling variation, the results 
for the 1:1 map ratio were identical to the corresponding scenarios in Figure 6 for the sex-averaged analysis (column 1, rows 1-3). With increasing map ratio, however, imprinting indices were significantly underestimated, especially in the case of smaller imprinting degrees $(I<0.8)$. This was due to maximization curves reaching into the left half-tetrahedron $\mathbf{T}_{\mathbf{z}_{1}^{\text {pat }}}>\mathbf{z}_{1}^{\text {mat }}$, as it was explained above (Results Section 3.3.4), which also applies to the maximization under imprinting. In the case of a map ratio $>1$, the corresponding variation in terms of $M A D$ was slightly higher for larger imprinting indices, compared to the corresponding sex-averaged MOBIT analysis (Figure 6, rows 1-3), but markedly lower for the scenarios with smaller $I$, in which imprinting indices were significantly underestimated. $M A D$ was comparable between the two sample sizes.
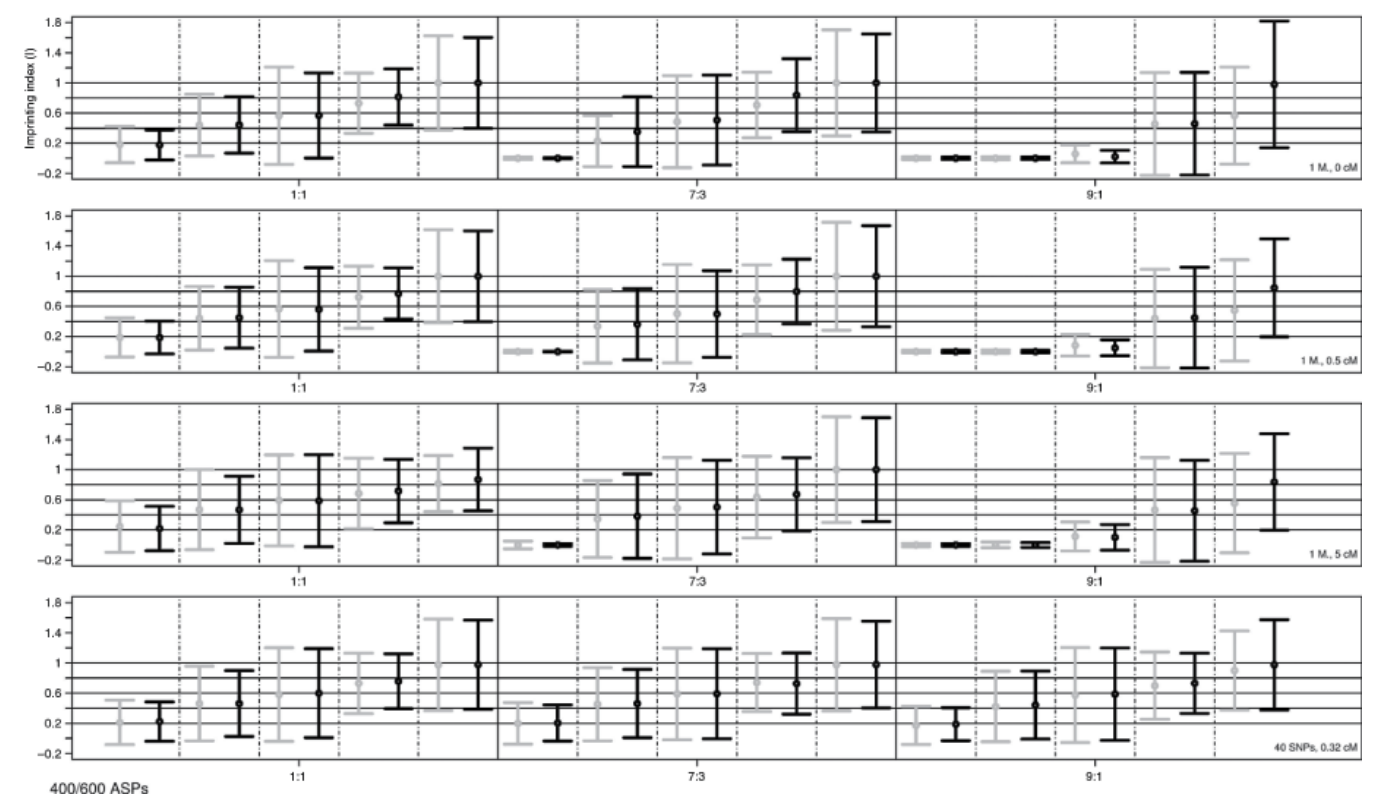

Figure 8: Estimation of imprinting index I by the MOBIT using a sex-specific map in the analysis of ASPs. For more details see Figure 3 and Figure 5.

The results of the imprinting parameter estimation using 3-G pedigrees are depicted in Figure 9 (rows 1-3). As with ASPs, results for the 1:1 map ratio with 3-G pedigrees were identical to the corresponding scenarios in Figure 7 for the sex-averaged analysis (column 1, rows 1-3). Estimated median imprinting indices were underestimated across all map ratios and marker-trait locus distances. $M A D$ increased from $I=0.2$ over $I=0.4$ to $I=0.6$, with comparable variation for $I \geq 0.6$. Further, $M A D$ slightly increased with larger marker-trait locus distance and map ratio. Compared to the results for ASPs (Figure 8, rows 1-3), a better imprinting parameter estimation accuracy in terms of lower bias and variability was obtained for 3-G pedigrees and map ratios $>1$. This was in accordance with the finding that 3-G pedigrees showed higher power than ASPs in a sex-specific MOBIT analysis for map ratios $>1$, probably due to a reduced effect of maximization curves (see Results Section 3.3.4). 

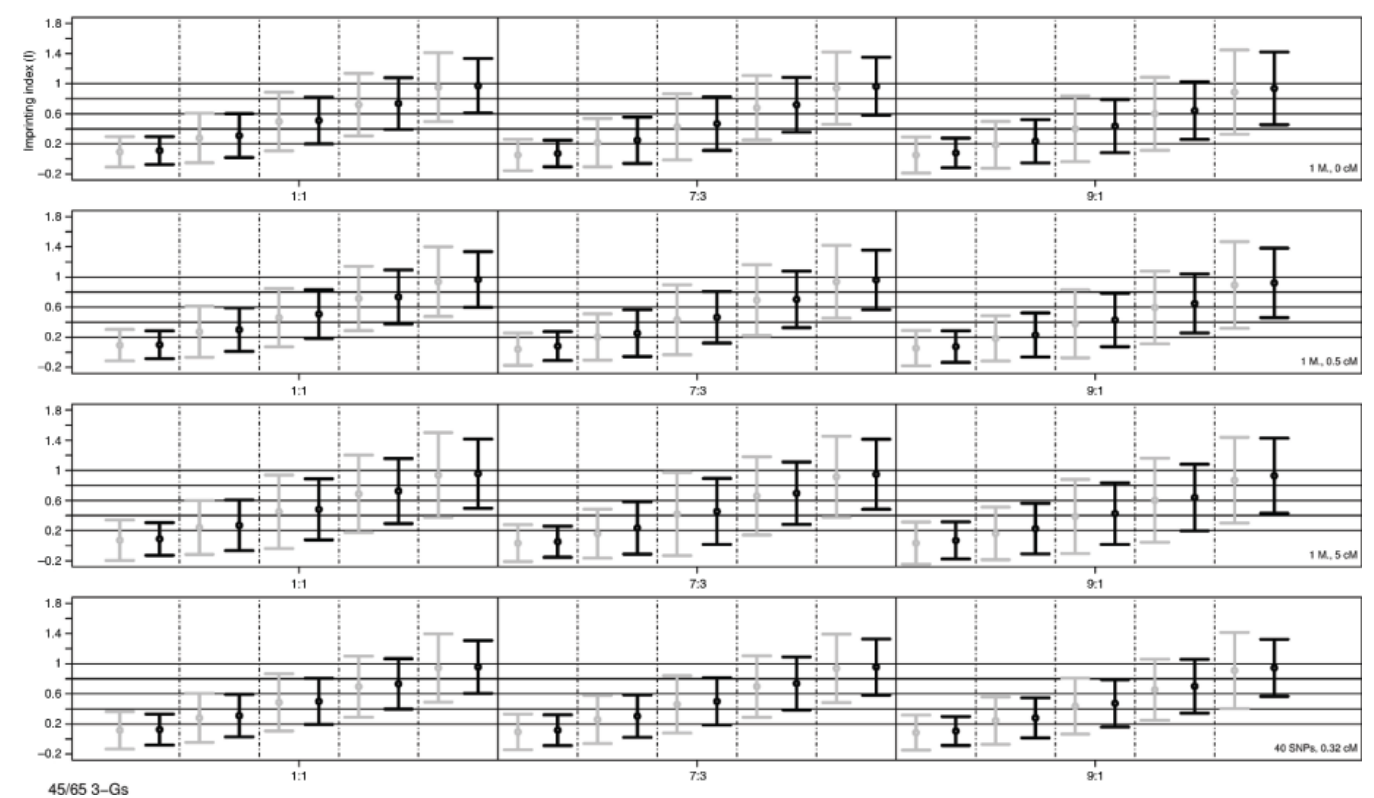

Figure 9: Estimation of imprinting index I by the MOBIT using a sex-specific map in the analysis of 3-G pedigrees. For more details see Figure 3 and Figure 5.

\subsubsection{Twopoint analysis, paternal imprinting}

The imprinting index estimation results for the paternal imprinting model using a sex-specific map in the analysis can be found in Figure 5 (right, rows 2 and 4) for both pedigree types. Estimation accuracy was generally better compared to the maternal imprinting scenario, especially for ASPs (see also Figure 8). This is due to a less distinguished effect of maximization curves, which particularly affects allele-sharing points in the other half tetrahedron $\mathbf{T}_{\mathbf{z}_{1}^{\text {pat }}>\mathbf{z}_{1}^{\text {mat }}}$. In the case of ASPs, imprinting indices for scenarios with $I<0.6$ were overestimated, whereas those with $I>0.6$ were underestimated. The corresponding results for 3-G pedigrees, however, were similar to the sex-averaged analysis due a less pronounced effect of maximization curves compared to ASPs.

\subsubsection{Multipoint analysis}

The results of the multipoint scenarios using a sex-specific map in the analysis for ASPs can be found in Figure 8 (row 4). The results were similar to the corresponding sex-averaged analyses (Figure 6, row 4). In particular, estimated median imprinting indices were close to their expected values across all map ratios. MAD was highest for $I=0.6$ and lowest for $I=0.2$. MAD was lower for the larger sample size for most scenarios and did not substantially differ between map ratios.

The multipoint results for 3-G pedigrees are shown in Figure 9 (row 4). As with ASPs, the results were similar to the corresponding sex-averaged analyses (Figure 7, row 4). In particular, imprinting indices were slightly underestimated across all map ratios. $M A D$ was lowest for $I=0.2$ and slightly increased with larger imprinting indices. Again, $M A D$ was lower for the larger sample size and was comparable with respect to different map ratios. In addition, MAD of the imprinting index was lower for 3-G pedigrees compared to ASPs (Figure 8, row 4).

\subsection{Real data example on house dust mite allergy}

The MOBIT results for all investigated chromosomes can be found in Table 6. Assuming an IMOD score larger than 4 to be an informative linkage signal, an empiric $p$ value was simulated using the two proposed MOBIT simulation/permutation procedures for the result on chromosome 8 near marker D8S511 (IMOD score $=4.9675$, MOD score $=3.186$, MOBIT $=1.7815$, best-fitting nonimprinting model: $p=0.07, f_{0}=0.0, f_{1}=0.0001, f_{2}=0.0$; best-fitting imprinting model: $p=0.14, f_{0}=0.002, f_{1, \text { pat }}=0.06, f_{1, \text { mat }}=0.0, f_{2}=0.0$, maternal imprinting). The empiric $\mathrm{p}$ value obtained using method bfnm was $\mathrm{p}=0.02$, whereas it was $\mathrm{p}=0.18$ using method perm. We also repeated the analysis using a sex-averaged map and obtained similar results (IMOD score $=5.1301$, 
MOD score $=3.2081$, MOBIT $=1.9219, \mathrm{p}=0.005$ using method bfnm and $\mathrm{p}=0.178$ using method perm). This finding illustrates that there may exist various ways to construct a valid simulation/permutation test, whereby the choice that maximizes power with simultaneous control over the type I error rate presumably depends on the underlying null hypothesis, the actual trait model and investigated pedigree types (see also Churchill and Doerges (2008)). In the case of the house dust mite data, method perm might have been less powerful than method bfnm. The corresponding results for the investigated scenarios in Appendix $C$ also showed differences between the two methods for both sex-averaged and sex-specific analyses. In the case of ASPs and when the correct sex-specific map was used in the analysis, differences might be due to the fact that the conditional null hypothesis underlying the perm method implies an invariant nonimprinting maximum likelihood estimate for the point in terms of allele-sharing ('preserved linkage'), such that there is no effect of maximization curves for the permuted replicates in a sex-specific nonimprinting MOD score analysis. Therefore, permuted replicates almost always lead to points in terms of allele-sharing that are exclusively reached by the IMOD score imprinting maximization (see Appendix Figure 11). As to the method bfnm, due to maximization curves, the nonimprinting maximum likelihood estimate has good chance to be close to the one obtained by the imprinting maximization for every simulated replicate, which leads to smaller quantiles of the null distribution compared to method perm.

Table 6: Results of the MOBIT real data application on house dust mite allergy using a sex-specific map in the analysis.

\begin{tabular}{|c|c|c|c|c|}
\hline Chromosome & Length in $\mathrm{cM}$ & MOD & IMOD & MOBIT \\
\hline 1 & 238.3 & 2.5933 & 2.5933 & 0 \\
\hline 2 & 240.8 & 1.9577 & 3.1872 & 1.2294 \\
\hline 3 & 147.7 & 0.8161 & 2.2488 & 1.4327 \\
\hline 4 & 153.6 & 3.7896 & 3.7991 & 0.0095 \\
\hline 5 & 140.3 & 3.1719 & 3.1771 & 0.0051 \\
\hline 6 & 154.4 & 2.4707 & 3.2292 & 0.7584 \\
\hline 7 & 124.9 & 2.772 & 3.2912 & 0.5193 \\
\hline 8 & 41.7 & 3.186 & 4.9675 & 1.7815 \\
\hline 9 & 0 & 0.602 & 0.602 & 0 \\
\hline 10 & 129.3 & 2.2501 & 2.5753 & 0.3253 \\
\hline 11 & 138.25 & 2.939 & 3.6833 & 0.7443 \\
\hline 12 & 101.4 & 1.5531 & 1.8384 & 0.2853 \\
\hline 13 & 87.9 & 2.8297 & 2.8297 & 0 \\
\hline 14 & 37.6 & 1.03 & 1.1569 & 0.1269 \\
\hline 15 & 53.8 & 1.572 & 1.6267 & 0.0548 \\
\hline 16 & 67.3 & 3.0966 & 3.1161 & 0.0195 \\
\hline 17 & 13.4 & 2.0128 & 2.0413 & 0.0286 \\
\hline 18 & 104.7 & 1.3458 & 1.3458 & 0 \\
\hline 19 & 58.5 & 1.526 & 2.1838 & 0.6578 \\
\hline 20 & 0 & 0.1522 & 0.1522 & 0 \\
\hline 21 & 27.5 & 1.055 & 2.076 & 1.021 \\
\hline
\end{tabular}

Length in cM: Length of the chromosomal segment covered by the typed markers. Values in bold indicate the most promising imprinting test result, for which an empiric $p$ value was calculated using the two proposed MOBIT simulation/permutation procedures.

\section{Discussion}

Linkage-based testing for genomic imprinting is a challenging task. This holds true for both parametric and nonparametric linkage analysis methods. In this paper, we proposed the likelihood ratio test statistic MOBIT as a new test for imprinting, which is based on the parametric MOD score approach (Clerget-Darpoux, BonaïtiPellié \& Hochez, 1986; Risch, 1984). The MOBIT is not restricted to the analysis of certain pedigree types, offers quantification of imprinting, does not assume independent parental meioses, and can readily be calculated using the GHM software package (Brugger \& Strauch, 2014; Dietter et al., 2007; Mattheisen et al., 2008; Strauch, 2003), which also allows usage of sex-specific maps in the analysis. Although the MOBIT can be considered as a canonical approach to test for imprinting in the presence of linkage, the null distribution of the MOBIT depends on the truly underlying but generally unknown mode of inheritance, i.e. disease allele frequency and penetrance function, which corresponds to a certain point in terms of allele-sharing within the allele-sharing parameter space of a given type of pedigree (see Figure 1 for the example of ASPs). We have shown that the MOBIT asymptotically follows a $\chi^{2}$ distribution with $1 \mathrm{df}$ irrespective of the pedigree type (see Appendix A.3). 
In the special case of no linkage, the MOBIT follows a mixture of distributions that includes non- $\chi^{2}$ components (see also Self and Liang (1987), case 8, pp. 608-609). As shown in this paper, this leads to quantiles that can be larger than those obtained assuming $\chi^{2}$ with $1 \mathrm{df}$ (see Table 3 for $3-G$ pedigrees). Generally, imprinting tests based on linkage test statistics are only advised in the case that linkage can be assumed to be present. But even in the presence of linkage, the quality of the asymptotic approximation of the MOBIT distribution strongly depends on the truly underlying mode of inheritance. If the true point in terms of allele-sharing lies near the boundary of the parameter space, MOBIT quantiles can be smaller or larger than those obtained assuming $\chi^{2}$ with $1 \mathrm{df}$. The degree of deviation from the asymptotic distribution directly depends on the structure of the parameter space of a given pedigree. Presumably, allele-sharing parameter spaces become more complicated with increasing pedigree complexity, which in turn means that more boundary conditions are to be expected with more complex pedigree structures. This assumption was underpinned by the present study, such that MOBIT quantiles for the additive trait model and the 1:1 map ratio corresponded to those expected assuming a $\chi^{2}$ distribution with $1 \mathrm{df}$ when ASPs were used in the analysis, whereas quantiles were clearly inflated when using 3-G pedigrees (Table 4 and Table 5, respectively). To circumvent the uncertainty about the quality of the asymptotic approximation of the MOBIT distribution, we proposed the ab initio simulation (method $b f n m$ ) of genotype data based on the best-fitting nonimprinting model obtained from the real dataset MOD score analysis. Alternatively, we developed a permutation procedure (method perm) similar to those proposed in Whittaker et al. (2003) and Dong et al. (2005), which generates replicates under the null hypothesis of no imprinting effect, conditional on the linkage information of the real dataset. During the permutation, the parental origin of transmitted alleles is randomized, while genotypes and hence linkage information are preserved (see Appendix C for more details). We have investigated the distributions of MOBIT scores using both methods bfnm and perm. However, both methods do not compensate for confounding (see Appendix Table 8). In addition, empiric quantiles and $p$ values differed between the two methods due to differences in the underlying null hypotheses and sample spaces. Using a real data example on house dust mite allergy, both methods to obtain empiric values were compared, which, however, led to different results. This finding indicates that differences in null hypotheses of the two tests may lead to different results and conclusions, especially for the analysis of complex pedigree data.

Another aspect in linkage-based imprinting testing is the confounding between genomic imprinting and sex-specific recombination fractions. This affects both parametric and nonparametric linkage methods. With respect to the MOBIT, type I error rates were increased under the null hypothesis of linkage but no imprinting $\left(H_{0, b}\right)$ due to confounding of imprinting and sex-specific recombination fractions in a sex-averaged twopoint analysis for both ASPs and 3-G pedigrees (top of Table 4 and Table 5, respectively, map ratios $>1$ ). Confounding was more severe for ASPs, the recessive trait model, the larger sample size, and the larger map ratio. In general, confounding is expected to be more severe for trait models with higher allele-sharing, such as the recessive model used for our simulations, because power to detect linkage and hence the potential of confounding is stronger in such a case. The same argument holds true for the larger sample size. In the case of ASPs, the $H_{0, b}$ distribution of the MOBIT for the 1:1 map ratio under the additive and recessive trait models did not show confounding and followed the assumed $\chi^{2}$ distribution with $1 \mathrm{df}$. In the case of 3-G pedigrees, the corresponding MOBIT quantiles were inflated for the additive trait model, probably because the true point in terms of allele-sharing lies on the boundary of the parameter space of 3-G pedigrees. In contrast, the MOBIT followed the assumed $\chi^{2}$ distribution with $1 \mathrm{df}$ for the recessive trait model, which was expected for non-boundary conditions, irrespective of the pedigree type (see Appendix A.3). Using a multipoint analysis avoided confounding as long as the marker spacing was sufficiently dense. We were able to show that a marker spacing of $1 \mathrm{cM}$ between two consecutive markers (marker-trait locus distance $0.5 \mathrm{cM}$ ) was sufficient to avoid confounding across both investigated pedigree types and all map ratios (top of Table 4 and Table 5). However, a marker spacing of $10 \mathrm{cM}$ between two consecutive markers (marker-trait locus distance $5 \mathrm{cM}$ ) led to confounding, with higher inflated type I error rates for the recessive trait model and for ASPs (top of Table 4 and Table 5).

With regard to the results of the sex-specific analyses (bottom of Table 4 and Table 5), type I error rates of the MOBIT were not inflated due to confounding for both pedigree types and trait models. However, type I error rates were deflated for the MOBIT for the twopoint scenarios and - to a lesser extent-for the 4 markers, $10 \mathrm{cM}$ scenario due to the problem of maximization curves as depicted in Figure 2. Interestingly, the effect of maximization curves was less pronounced for 3-G pedigrees, which indicates differences in the parameter spaces between nuclear families and extended pedigrees. In addition, the effect of maximization curves seemed to be slightly more severe for the recessive trait model, although this was only seen for ASPs (bottom of Table 4).

Apart from the type I error rate, we also assessed the corresponding power of the MOBIT to detect imprinting. As expected, power to detect imprinting was higher for the larger sample size in all scenarios. In the case of a map ratio of 1:1, power was generally higher for ASPs (Figure 4 and Figure 5). With regard to the power calculations when using a sex-averaged map for the analysis, the MOBIT had reasonable power to detect im- 
printing across all investigated marker scenarios, map ratios, and pedigree types (Figure 4 and Figure 5). The power of the MOBIT only slightly depended on the truly underlying map ratio for the 1 marker, $5 \mathrm{cM}$ scenario and - to a lesser extent-for the 40 SNPs, $0.32 \mathrm{cM}$ scenario for both pedigree types. Due to the maximization curve problem, the MOBIT had almost no power in the sex-specific twopoint analyses with map ratios $>1$ using ASPs for the maternal imprinting model (second row of Figure 4). With 3-G pedigrees, however, the MOBIT had markedly better power for all map ratios than with ASPs (last row of Figure 4). With regard to the multipoint results using $40 \mathrm{SNPs}$, the MOBIT showed good power to detect imprinting for both pedigree types. Using a paternal imprinting model, in which the nonimprinted sex now has the longer genetic map, the power to detect imprinting was reasonably high for sex-averaged and sex-specific analyses as well as for both pedigree types (Figure 5).

Although the power to detect imprinting is bounded from above by the power to detect linkage, this does not imply that the power to detect imprinting must be similar for different pedigrees showing similar power to detect linkage. Put another way, imprinting information is not equivalent to linkage information. Along these lines, ASPs had generally greater power to detect imprinting than the 3-G pedigrees used in our simulations, although the datasets for both pedigree types contained equal amounts of linkage information due to adjusted sample sizes. This is because the 3-G pedigree was constructed such that, for maternal imprinting, a considerable proportion of phenocopies and/or carriers of maternally inherited mutant alleles were simulated as affected individuals 8 and 9, because of the mother individual 5 propagating the mutant allele down the pedigree (see Figure 3B). The mutant allele might also have entered the pedigree through founder individual 3 (spouse of mother individual 5), although this being less likely in regard of the rather small disease allele frequency of $p=0.1$.

Using MOD scores, it should in principle be possible to obtain unbiased estimates of the trait-model parameters $f_{0}, f_{1, \text { pat }}, f_{1, \text { mat }}, f_{2}$, and $\mathrm{p}$ (Elston, 1989). This is due to the fact that the likelihood ratio in the MOD score corresponds to the conditional probability of observing the marker data given the trait phenotypes (ClergetDarpoux, Bonaïti-Pellié \& Hochez, 1986). However, the identifiability of the trait-model parameters actually depends on the number of free parameters in terms of allele-sharing classes of the investigated pedigree type(s). With ASPs, the allele-sharing classes are $z_{0}, z_{1}^{\text {pat }}, z_{1}^{\text {mat }}$, and $z_{2}$ when taking imprinting into account. Hence, as there are only $4-1=3$ free parameters that can be estimated from ASP data, there will be many sets of $f_{0}, f_{1 \text { pat }}, f_{1 \text { mat }}, f_{2}, \mathrm{p}$, and $\theta$ that correspond to the estimated $z_{0}, z_{1}^{\text {pat }}, z_{1}^{\text {mat }}$, and $z_{2}$. With larger pedigrees, and hence more allele-sharing classes, the degree to which the trait-model parameters can be determined should be higher. Further information reffering to the ability of a MOD score analysis to correctly determine the truly underlying trait-model parameters for different pedigree types can be found in Brugger, Rospleszcz, and Strauch (2016). Here, we were interested in the ability of a MOBIT analysis to correctly estimate the degree of imprinting, as defined by the imprinting index I (Strauch, 2005). As a result, the estimated median imprinting indices of the two- and multipoint analyses assuming a sex-averaged map were close to their expected values for ASPs and the maternal imprinting model, except the 1 marker, $5 \mathrm{cM}$ scenario, for which indices were overestimated when $<0.6$ and underestimated when $\geq 0.6$ (Figure 6 ). In the case of $3-G$ pedigrees, imprinting indices for the maternal imprinting model were mostly close to their expected values, although always underestimated (Figure 7). In the case of the paternal imprinting model, estimates of the imprinting index were often underestimated for both pedigree types due to the longer genetic map of the nonimprinted sex (Figure 5). The results of the sex-specific analyses for ASPs and the maternal imprinting model in Figure 8 indicated that the estimates were biased towards lower values due to the maximization curve problem, which is in line with the reduced power values shown in Figure 4. The estimates for the 3-G pedigrees, however, were only slightly different from those of the corresponding sex-averaged analysis (Figure 9). Specifically, estimated median imprinting indices were again always underestimated, but to a slightly more severe degree than it was for the sex-averaged analysis. In contrast, in the case of the paternal imprinting model, imprinting indices could be estimated more accurately using the correct sex-specific map in the analysis for both pedigree types (Figure 5). Generally, imprinting indices could be estimated more accurately using ASP pedigrees, because they harbour more imprinting information compared to the 3-G pedigrees studied here, although power to detect linkage was similar for both pedigree types (see also explanation above).

Referring to the question whether to opt for a two- or multipoint approach in a MOBIT imprinting analysis, multipoint analysis should generally be preferred, because it showed good power irrespective of the truly underlying map ratio and whether or not the correct sex-specific map was used in the analysis (see Figure 4). Moreover, quantification of imprinting in terms of the imprinting index $I$ is more reliable in the multipoint setting, especially when marker spacing is dense.

It is of note, however, that differences in heterozygote penetrances might also be caused by another parentof-origin effect, namely maternal effects. Maternal effects refer to the phenotype of an individual being influenced by the genotype of the mother (Han, Hu \& Lin, 2013), whereby it can induce the same phenotypic pattern in the offspring as genomic imprinting (Hager, Cheverud \& Wolf, 2008). Hence, maternal effects and genomic 
imprinting are confounded and cannot be distinguished using the MOBIT or any other existing nonparametric linkage method by looking at different heterozygote penetrances or parental allele-sharing values, respectively. A way to separate maternal effects from genomic imprinting has been proposed for parent-offspring data and was investigated in the context of quantitative trait loci by Hager, Cheverud, and Wolf (2008). An extension of the MOD score approach that allows to distinguish maternal effects from imprinting would be an interesting future research item, because this would further refine the possibility of the MOD score approach to characterize the disease gene variant, based on trait-model parameter estimates as shown by Brugger, Rospleszcz, and Strauch (2016).

In this work, we have proposed the new imprinting test statistic MOBIT and evaluated its properties using extensive simulations. With regard to the effect of confounding, the MOBIT showed inflated type I error rates when the marker spacing was not dense enough $(>1 \mathrm{cM})$, which can be remedied by using sex-specific maps. However, sex-specific twopoint analyses should be avoided, because the test for imprinting has no power when the underlying genetic map ratio is large, which is due to the so-called maximization curve problem. When a sufficiently dense marker map with a marker spacing of less than $1 \mathrm{cM}$ is used in the analysis, the difference between sex-specific and sex-averaged maps is marginal, if not negligible. In such a case, the MOBIT did not show an effect of confounding, had good power to detect imprinting, and was able to reliably estimate the degree of imprinting, the accuracy of which depended on the pedigree type used in the analysis. Hence, we recommend the usage of sufficiently dense marker frameworks to avoid both confounding of sex-specific recombination fractions and imprinting as well as low power due to the maximization curve problem. In addition, we proposed two alternative simulation/permutation methods to obtain empiric $p$ values for the MOBIT and compared them using a real dataset and various scenarios of the main simulation study. In general, we recommend to apply the method $b f n m$, which relies on the fully parametric ab initio simulation of genotypes according to the best-fitting nonimprinting model obtained from the real dataset analysis, because it is the canonical approach to generate replicates under the null hypothesis of linkage, but no imprinting. Replicates for the method $b f n m$ can readily be generated using the SLINK software package (Ott, 1989; Weeks et al., 1990; Schäffer et al., 2011). However, there might be situations in which the null hypothesis of method perm, i.e. no imprinting effect, conditional on the linkage information of the real dataset, might also prove useful, e.g. when the research interest lies in testing a null hypothesis that is confined to the exact realisation of the real dataset. We implemented the perm method in a new version of the GHM software package. Taken together, the MOBIT is a recommendable tool to discover new imprinted loci and offers good flexibility with regard to marker scenarios and pedigree types.

The imprinting test statistic MOBIT and a corresponding $\mathrm{p}$ value according to the perm method can be calculated using the GENEHUNTER-MODSCORE program, which has recently been optimized to provide a fast linkage analysis with evaluation of many sets of trait-model parameters by algebraic computations (Brugger \& Strauch, 2014). The program can be freely downloaded from our website http://www.helmholtzmuenchen.de/ige/service/software-download/index.html.

\section{Acknowledgement}

This work was supported by grants Str643/4-1 and Str643/6-1 of the Deutsche Forschungsgemeinschaft (German Research Foundation). Further, this research was supported within the Munich Center of Health Sciences (MC-Health), Ludwig-Maximilians-Universität, as part of LMUinnovativ. The authors gratefully acknowledge the Gauss Centre for Supercomputing e.V. (www.gauss-centre.eu) for funding this work by providing computing time on the GCS Supercomputer SuperMUC at Leibniz Supercomputing Centre (www.lrz.de). In addition, the authors are grateful for the computing resources on the High Performance Computing - High Availability - Cluster (HPC-HA-Cluster) provided by the Helmholtz Zentrum München. Finally, we would like to thank the reviewers for their thoughtful comments that helped us to substantially improve the manuscript.

\section{Funding}

Deutsche Forschungsgemeinschaft, Funder Id: http://dx.doi.org/10.13039/501100001659, Grant Number: Str643/4-1 and Str643/6-1. 


\section{A Appendix}

\section{A.1 Proof of the equivalence of the nonimprinting and imprinting likelihood in the test for imprinting using affected sib-pairs (ASPs) in the case of no linkage}

An ASP with arbitrary genotype information (missing or non-missing) for parents and offspring is given. Without loss of generality, allele sharing at a single marker is considered. The following equality is to be shown:

$$
\prod_{i=1}^{n}\left(w_{i 0} \cdot \frac{1}{4}+w_{i 1} \cdot \frac{1}{2}+w_{i 2} \cdot \frac{1}{4}\right)=\prod_{i=1}^{n}\left(w_{i 0} \cdot \frac{1}{4}+w_{i 1^{p a t}} \cdot \frac{1}{4}+w_{i 1^{\text {mat }}} \cdot \frac{1}{4}+w_{i 2} \cdot \frac{1}{4}\right)
$$

with

$$
w_{i j}=P\left(g_{\text {off }, i} \mid i b d_{i}=j\right)=\frac{P\left(i b d_{i}=j \mid g_{\text {off }, i}\right) \cdot P\left(g_{\text {off }, i}\right)}{P\left(i b d_{i}=j\right)}
$$

- $P\left(g_{o f f}, i\right)$ : probability of genotypes of both offspring for the $i$-th ASP

- pat/mat: determines parental, i.e. paternal or maternal, origin of the shared allele in the imprinting likelihood formulation

- $P\left(i b d_{i}=j\right.$ ): probability of sharing $j=0,1,2$ (or 0, $1^{\text {pat }}, 1^{\text {mat }}, 2$ with imprinting) alleles identical-by-descent (IBD) for the $i$-th ASP under the null hypothesis of no linkage, which is $\left\{\frac{1}{4}, \frac{1}{2}, \frac{1}{4}\right\}$ for the nonimprinting and $\left\{\frac{1}{4}, \frac{1}{4}, \frac{1}{4}, \frac{1}{4}\right\}$ for the imprinting likelihood, respectively

- $P\left(i b d_{i}=j \mid g_{\text {off },}\right)$ : probability of the $i$-th ASP sharing $j=0,1,2$ (or $0,1^{\text {pat }}, 1^{\text {mat }}, 2$ with imprinting) alleles IBD, conditional on offspring genotypes, which is $\left\{z_{i 0}, z_{i 1}, z_{i 2}\right\}$ for the nonimprinting and $\left\{z_{i 0}, z_{i 1}^{\text {pat }}, z_{i 1}^{\text {mat }}, z_{i 2}\right\}$ for the imprinting likelihood, respectively

In order to show that equation (3) holds, it is sufficient to show that the corresponding equality holds for each ASP:

$$
w_{i 0} \cdot \frac{1}{4}+w_{i 1} \cdot \frac{1}{2}+w_{i 2} \cdot \frac{1}{4}=w_{i 0} \cdot \frac{1}{4}+w_{i 1^{p a t}} \cdot \frac{1}{4}+w_{i 1^{\text {mat }}} \cdot \frac{1}{4}+w_{i 2} \cdot \frac{1}{4}
$$

Given the assumption that $w_{i 0}$ and $w_{i 2}$ do not change when the case of $i b d=1$ shared allele is distinguished according to its parental origin, this reduces to:

$$
\begin{aligned}
w_{i 1} \cdot \frac{1}{2} & =w_{i 1^{\text {pat }}} \cdot \frac{1}{4}+w_{i 1^{\text {mat }}} \cdot \frac{1}{4} \\
w_{i 1} & =\frac{w_{i 1^{\text {pat }}}+w_{i 1^{\text {mat }}}}{2}
\end{aligned}
$$

Using equation (4), we can write for the right side of equation (6):

$$
\begin{aligned}
w_{i 1^{\text {pat }}}+w_{i 1^{\text {mat }}} & =\frac{P\left(i b d_{1}=1^{\text {pat }} \mid g_{\text {off, } 1}\right) \cdot P\left(g_{\text {off, } 1}\right)}{\frac{1}{4}} \\
& +\frac{P\left(i b d_{1}=1^{\text {mat }} \mid g_{\text {off, } 1}\right) \cdot P\left(g_{\text {off, } 1}\right)}{\frac{1}{4}} \\
& =4 \cdot P\left(g_{\text {off, } 1}\right) \cdot\left\{P\left(i b d_{1}=1^{\text {pat }} \mid g_{\text {off, } 1}\right)+P\left(i b d_{1}=1^{\text {mat }} \mid g_{\text {off, } 1}\right)\right\} \\
& =4 \cdot P\left(g_{\text {off, } 1}\right) \cdot P\left(i b d_{1}=1 \mid g_{\text {off, } 1}\right)
\end{aligned}
$$

The last step holds because $z_{11}=z_{11}^{\text {pat }}+z_{11}^{\text {mat }}$. For $w_{i 1}$, we get:

$$
\begin{aligned}
w_{i 1} & =\frac{P\left(i b d_{1}=1 \mid g_{\text {off, } 1}\right) \cdot P\left(g_{\text {off }, 1}\right)}{\frac{1}{2}} \\
& =2 \cdot P\left(g_{\text {off, } 1}\right) \cdot P\left(i b d_{1}=1 \mid g_{\text {off }, 1}\right)
\end{aligned}
$$


Replacing last line of equation (7) and last line of equation (8) into the last line of equation (6), we get:

$$
2 \cdot\left\{2 \cdot P\left(g_{\text {off, } 1}\right) \cdot P\left(i b d_{1}=1 \mid g_{\text {off, } 1}\right)\right\}=4 \cdot P\left(g_{\text {off, } 1}\right) \cdot P\left(i b d_{1}=1 \mid g_{\text {off, } 1}\right)
$$

Since this holds in general for each ASP, it follows that equation (3) holds for the entire dataset with any number of ASPs.

\section{A.2 Proof of the equivalence of the nonimprinting and imprinting likelihood in the test for imprinting using arbitrary pedigrees in the case of no linkage}

An arbitrary pedigree type with arbitrary genotype information (missing or non-missing) for founders and nonfounders is given. Without loss of generality, allele-sharing at a single marker is considered. The numbers of shared alleles IBD for affected relative pairs are generalized to allele-sharing classes for arbitrary pedigree structures (see also Brugger and Strauch (2014)). The following equality is to be shown:

$$
\begin{gathered}
\prod_{i=1}^{n} \sum_{j=0}^{j \in \mathscr{V}_{\text {nonimp }}}\left(w_{i j} \cdot P\left(i b d_{i}=j\right)\right)=\prod_{i=1}^{n} \sum_{j=0}^{j \in \mathscr{V}_{\text {imp }}}\left(w_{i j} \cdot P\left(i b d_{i}=j\right)\right) \\
w_{i j}=P\left(g_{\text {off }, i} \mid i b d_{i}=j\right)=\frac{P\left(i b d_{i}=j \mid g_{\text {off }, i}\right) \cdot P\left(g_{\text {off }, i}\right)}{P\left(i b d_{i}=j\right)}
\end{gathered}
$$

with $\mathscr{V}_{\text {nonimp }}$ denoting the set of allele-sharing classes without imprinting and $\mathscr{V}_{\text {imp }}$ denoting the set of allelesharing classes with imprinting.

- $P\left(g_{o f f, i}\right)$ : probability of genotypes of all offsprings for the $i$-th pedigree

- $P\left(i b d_{i}=j\right)$ : probability of allele-sharing class $j$ for the $i$-th pedigree under the null hypothesis of no linkage, which is a constant value

- $P\left(i b d_{i}=j \mid g_{o f f}, i\right)$ : probability of allele-sharing class $j$ for the $i$-th pedigree, conditional on offspring genotypes

In order to show that equation (10) holds, it is sufficient to show that the corresponding equality holds for each pedigree:

$$
\sum_{j=0}^{j \in \mathscr{V}_{\text {nonimp }}}\left(w_{i j} \cdot P\left(i b d_{i}=j\right)\right)=\sum_{j=0}^{j \in \mathscr{T}_{\text {imp }}}\left(w_{i j} \cdot P\left(i b d_{i}=j\right)\right)
$$

For those allele-sharing classes that are identical with and without imprinting, i.e. those contained in $\mathscr{V}_{\text {nonimp }} \cap$ $\mathscr{V}_{i m p}$, the corresponding $w_{i j}$ are identical. Hence, equation (12) reduces to:

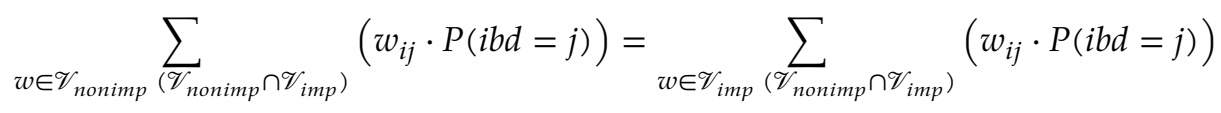

We now plug in equation (11) into equation (13) to get the following:

$$
\begin{gathered}
\sum_{w \in \mathscr{V}_{\text {nonimp }}} \sum_{\left.w \in \mathscr{V}_{\text {nonimp }} \cap \mathscr{V}_{\text {imp }}\right)}\left(\frac{P\left(i b d=j \mid g_{\text {off }}\right) \cdot P\left(g_{\text {off }}\right)}{P(i b d=j)} \cdot P(i b d=j)\right) \\
\sum_{\left(\mathscr{V}_{\text {nonimp }} \cap \mathscr{V}_{\text {imp }}\right)}\left(\frac{P\left(i b d=j \mid g_{\text {off }}\right) \cdot P\left(g_{\text {off }}\right)}{P(i b d=j)} \cdot P(i b d=j)\right) \\
=\sum_{w \in \mathscr{V}_{\text {nonimp }}} \sum_{\left(\mathscr{V}_{\text {nonimp }} \cap \mathscr{V}_{\text {imp }}\right)}\left(P\left(i b d=j \mid \mathscr{V}_{\text {off }}\right) \cdot P\left(g_{\text {off }}\right)\right) \\
\left(\mathscr{V}_{\text {nonimp }} \cap \mathscr{V}_{\text {imp }}\right)
\end{gathered}
$$




$$
\begin{aligned}
& P\left(g_{\text {off }}\right) \cdot \sum_{w \in \mathscr{V}_{\text {nonimp }}} \sum_{\left(\mathscr{V}_{\text {nonimp }} \cap \mathscr{V}_{\text {imp }}\right)} P\left(i b d=j \mid g_{\text {off }}\right) \\
& =P\left(g_{\text {off }}\right) \cdot \sum_{w \in \mathscr{V}_{\text {imp }}} P\left(i b d=j \mid g_{\text {off }}\right) \\
& \sum_{\left.w \in \mathscr{V}_{\text {nonimp }} \cap \mathscr{V}_{\text {imp }}\right),} P\left(i b d=j \mid g_{\text {off }}\right) \\
& =\sum_{w \in \mathscr{V}_{\text {imp }}}\left(\sum_{\left.\mathscr{V}_{\text {nonimp }} \cap \mathscr{V}_{\text {imp }}\right)} P\left(i b d=j \mid g_{\text {off }}\right)\right.
\end{aligned}
$$

Given that the sum of IBD probabilities of non-overlapping allele-sharing classes without imprinting must equal the corresponding sum with imprinting, the likelihoods are identical. This also extends to datasets containing mixtures of different pedigree types.

\section{A.3 Proof that the MOBIT follows a $\chi^{2}$ distribution with 1 degree of freedom under the null hypothesis of linkage but no imprinting for arbitrary pedigrees}

Given the proof in Appendix A.2, the MOBIT for arbitrary pedigree types, corresponding to equation (1) in the Methods section for the special case of ASPs, can also be written as a likelihood ratio of the nonparametric likelihoods taking imprinting into account (numerator) vs. not taking imprinting into account (denominator). The nonparametric allele-sharing probabilities $\mathbf{z}$ can be further expressed as functions of the penetrances, the disease allele frequency, and the recombination fraction (see also Brugger and Strauch (2014)):

$$
\begin{aligned}
\text { MOBIT } & =\log _{10} \frac{L\left(\hat{\mathbf{z}}_{\text {imp }}\right)}{L\left(\hat{\mathbf{z}}_{\text {nonimp }}\right)} \\
& =\log _{10} \frac{L\left(\mathbf{z}_{\mathbf{i m p}}\left(\hat{f}_{0}, \hat{f}_{1 \text { pat }}, \hat{f}_{1 \text { mat }}, \hat{f_{2}}, \hat{p}, \hat{\theta}\right)\right)}{L\left(\mathbf{z}_{\text {nonimp }}\left(\hat{f}_{0}, \hat{f}_{1}, \hat{f_{2}}, \hat{p}, \hat{\theta}\right)\right)} \\
& =\log _{10} \frac{L\left(\hat{f}_{0}, \hat{f}_{1 \text { pat }}, \hat{f}_{1 \text { mat }}, \hat{f_{2}}, \hat{p}, \hat{\theta}\right)}{L\left(\hat{f}_{0}, \hat{f}_{1}, \hat{f_{2}}, \hat{p}, \hat{\theta}\right)}
\end{aligned}
$$

, where $\hat{\mathbf{z}}_{\mathbf{i m p}}, \hat{\mathbf{z}}_{\text {nonimp }}, \hat{f}_{0}, \hat{f}_{1}, \hat{f}_{1 \text { pat }}, \hat{f}_{1 \text { mat }}, \hat{f_{2}}, \hat{p}, \hat{\theta}$ denote the maximum likelihood estimators (MLEs) of the respective parameters. Likelihood ratio theory tells us that the ratio in equation (18) follows a $\chi^{2}$ distribution with the number of degrees of freedom equal to the difference of independently maximized parameters in the numerator vs. the denominator (Wilks, 1938), which equals 1 in our case. Further, the hypothesis in the denominator should be a nested composite hypothesis of the one in the numerator (Wilks, 1938), which is true, because fixing $f_{1, \text { pat }}=f_{1, \text { mat }}$ in the numerator corresponds to the likelihood in the denominator. It is of note, however, that the asymptotic distribution no longer holds in the case of boundary conditions with respect to the alternative hypothesis in the numerator (Self \& Liang, 1987). In such a case, the empiric MOBIT distribution quantiles can be lower or higher than those expected for a $\chi_{1}^{2}$ distribution.

\section{B Appendix}

B.1 Proof that the marker-trait locus distance is identifiable in a MOD score analysis using ASPs if: i) sex-specific recombination frequencies are present and used in the analysis; and ii) imprinting is truly absent

Let $\left(\tilde{f}_{2}, \tilde{f}_{1, \text { pat }}, \tilde{f}_{1, \text { mat }}, \tilde{f}_{0}, \tilde{p}\right)$ and $\left(f_{2}^{*}, f_{1 \text { pat }}^{*}, f_{1 \text { mat }}^{*}, f_{0}^{*}, p^{*}\right)$ be two genetic disease models without imprinting, i.e. $\tilde{f}_{1, \text { pat }}=\tilde{f}_{1, \text { mat }}$ and $f_{1 \text { pat }}^{*}=f_{1 \text { mat }}^{*}$, and $\tilde{u}=\left(\tilde{u}_{2}, \tilde{u}_{1}^{\text {pat }}, \tilde{u}_{1}^{\text {mat }}, \tilde{u}_{0}\right) \neq(1 / 4,1 / 4,1 / 4,1 / 4)$ and $u^{*}=$ 
$\left(u_{2}^{*}, u_{1}^{* \text { pat }}, u_{1}^{* m a t}, u_{0}^{*}\right) \neq(1 / 4,1 / 4,1 / 4,1 / 4)$ the corresponding IBD distributions at the disease locus with $\tilde{u}_{1}^{\text {pat }}=\tilde{u}_{1}^{\text {mat }}$ and $u_{1}^{* \text { pat }}=u_{1}^{* m a t}$. Further, $\tilde{\theta}_{m} \neq \tilde{\theta}_{f}$ and $\theta_{m}^{*} \neq \theta_{f}^{*}$ are sex-specific recombination frequencies ( $m$ : male; $f$ : female) and $\tilde{z}$ and $z^{*}$ are the IBD distributions at the marker locus that are induced by the sex-specific recombination frequencies. Proposition: From $\tilde{z}=z^{*}$, it follows:

$$
C_{f}^{*}=\frac{\tilde{C}_{f}}{\tilde{C}_{m}} C_{m}^{*}
$$

with $\tilde{C}_{f}:=\tilde{\theta}_{f}^{2}+\left(1-\tilde{\theta}_{f}\right)^{2}-0.5, \tilde{C}_{m}:=\tilde{\theta}_{m}^{2}+\left(1-\tilde{\theta}_{m}\right)^{2}-0.5$ and $C_{f}^{*}:=\theta_{f}^{* 2}+\left(1-\theta_{f}^{*}\right)^{2}-0.5, C_{m}^{*}:=\theta_{m}^{* 2}+\left(1-\theta_{m}^{*}\right)^{2}-0.5$ Proof:

With

$$
\begin{aligned}
& T\left(\psi_{m}, \psi_{f}\right)= \\
& \left(\begin{array}{cccc}
\psi_{m} \psi_{f} & \psi_{m}\left(1-\psi_{f}\right) & \left(1-\psi_{m}\right) \psi_{f} & \left(1-\psi_{m}\right)\left(1-\psi_{f}\right) \\
\psi_{m}\left(1-\psi_{f}\right) & \psi_{m} \psi_{f} & \left(1-\psi_{m}\right)\left(1-\psi_{f}\right) & \left(1-\psi_{m}\right) \psi_{f} \\
\left(1-\psi_{m}\right) \psi_{f} & \left(1-\psi_{m}\right)\left(1-\psi_{f}\right) & \psi_{m} \psi_{f} & \psi_{m}\left(1-\psi_{f}\right) \\
\left(1-\psi_{m}\right)\left(1-\psi_{f}\right) & \left(1-\psi_{m}\right) \psi_{f} & \psi_{m}\left(1-\psi_{f}\right) & \psi_{m} \psi_{f}
\end{array}\right)
\end{aligned}
$$

we have $\tilde{z}=T\left(\tilde{\psi}_{m}, \tilde{\psi}_{f}\right) \cdot \tilde{u}$ and hence

$$
\begin{aligned}
\tilde{z}_{1}^{p a t}-\tilde{z}_{1}^{m a t} & =\left(\tilde{\psi}_{m}-\tilde{\psi}_{f}\right) \cdot\left(\tilde{u}_{2}-\tilde{u}_{0}\right), \\
\tilde{z}_{2}-\tilde{z}_{0} & =\left(\tilde{\psi}_{m}+\tilde{\psi}_{f}-1\right) \cdot\left(\tilde{u}_{2}-\tilde{u}_{0}\right) .
\end{aligned}
$$

Let now be $S\left(\psi_{m}, \psi_{f}\right)=$

$$
\left(\begin{array}{cccc}
\psi_{m} \psi_{f} & -\psi_{m}\left(1-\psi_{f}\right) & -\left(1-\psi_{m}\right) \psi_{f} & \left(1-\psi_{m}\right)\left(1-\psi_{f}\right) \\
-\psi_{m}\left(1-\psi_{f}\right) & \psi_{m} \psi_{f} & \left(1-\psi_{m}\right)\left(1-\psi_{f}\right) & -\left(1-\psi_{m}\right) \psi_{f} \\
-\left(1-\psi_{m}\right) \psi_{f} & \left(1-\psi_{m}\right)\left(1-\psi_{f}\right) & \psi_{m} \psi_{f} & -\psi_{m}\left(1-\psi_{f}\right) \\
\left(1-\psi_{m}\right)\left(1-\psi_{f}\right) & -\left(1-\psi_{m}\right) \psi_{f} & -\psi_{m}\left(1-\psi_{f}\right) & \psi_{m} \psi_{f}
\end{array}\right)
$$

$$
\begin{aligned}
& \text { It can be shown that } T^{-1}\left(\tilde{\psi}_{m}, \tilde{\psi}_{f}\right)=\frac{1}{\left(\psi_{m}^{2}-\left(1-\psi_{m}\right)^{2} \cdot\left(\psi_{f}^{2}-\left(1-\psi_{f}\right)^{2}\right)\right.} \cdot S\left(\psi_{m}, \psi_{f}\right) \text {. Due to } \tilde{z}=z^{*} \text { we have } u^{*}= \\
& 0=u_{1}^{* p a t}-u_{1}^{* m a t}=\left(\psi_{f}^{*}-\psi_{m}^{*}\right) \cdot\left(\tilde{z}_{2}-\tilde{z}_{0}\right)+\left(\psi_{f}^{*}+\psi_{m}^{*}-1\right) \cdot\left(\tilde{z}_{1}^{\text {pat }}-\tilde{z}_{1}^{\text {mat }}\right) \\
& =\left[\left(\psi_{f}^{*}-\psi_{m}^{*}\right) \cdot\left(\tilde{\psi}_{m}+\tilde{\psi}_{f}-1\right)-\left(\psi_{f}^{*}+\psi_{m}^{*}-1\right) \cdot\left(\tilde{\psi}_{f}-\tilde{\psi}_{m}\right)\right] \cdot\left(\tilde{u}_{2}-\tilde{u}_{0}\right) \\
& =\left[\psi_{f}^{*} \cdot\left(2 \tilde{\psi}_{m}-1\right)-\psi_{m}^{*} \cdot\left(2 \tilde{\psi}_{f}-1\right)+\left(\tilde{\psi}_{f}-\tilde{\psi}_{m}\right)\right] \cdot\left(\tilde{u}_{2}-\tilde{u}_{0}\right) \\
& =2\left(C_{f}^{*} \cdot \tilde{C}_{m}-C_{m}^{*} \cdot \tilde{C}_{f}\right) \cdot\left(\tilde{u}_{2}-\tilde{u}_{0}\right)
\end{aligned}
$$

Due to $u \neq(1 / 4,1 / 4,1 / 4,1 / 4)$ we have $\tilde{u}_{2}-\tilde{u}_{0}>0$, which leads to proposition (19). It remains to be shown that for two pairs $\left(\tilde{\theta}_{m}, \tilde{\theta}_{f}\right)$ and $\left(\theta_{m}^{*}, \theta_{f}^{*}\right)$, for which equation (19) holds and for which the ratios of genetic map distances (using the Haldane mapping function) are identical, $\tilde{\theta}_{m}=\theta_{m}^{*}$ and $\tilde{\theta}_{f}=\theta_{f}^{*}$ is always true. To this end, it should be noted that the range of values for $C(\theta)=\theta^{2}+(1-\theta)^{2}-0.5$ corresponds to the interval $(0,1 / 2)$ if $\theta \in(0,1 / 2)$ and that $C((1-\sqrt{2 U}) / 2)=U$. Hence, for the genetic distance $x_{f}^{*}$ corresponding to $\theta_{f}^{*}$ we have

$$
\begin{aligned}
x_{f}^{*} & =-0.5 \ln \left(1-2 \cdot\left(1-\sqrt{2 C_{f}^{*}}\right) / 2\right)=-0.25 \log \left(2 C_{f}^{*}\right) \text { and } \\
\frac{x_{f}^{*}}{x_{m}^{*}} & =\frac{\log 2 C_{f}^{*}}{\log 2 C_{m}^{*}} .
\end{aligned}
$$


With $\tilde{a}:=\tilde{C}_{f} / \tilde{C}_{m}$ we hence get

$$
\frac{x_{f}^{*}}{x_{m}^{*}}=\frac{\log 2 \tilde{a} C_{m}^{*}}{\log 2 C_{m}^{*}}
$$

if $\left(\theta_{m}^{*}, \theta_{f}^{*}\right)$ fulfill equation (19).

In the same line,

$$
\frac{\tilde{x}_{f}}{\tilde{x}_{m}}=\frac{\log 2 a^{*} \tilde{C}_{m}}{\log 2 \tilde{C}_{m}}
$$

holds with $a^{*}:=C_{f}^{*} / C_{m}^{*}$ and $\tilde{a}=a^{*}:=a$ due to equation (19).

If then

$$
\frac{x_{f}^{*}}{x_{m}^{*}}=\frac{\tilde{x}_{f}}{\tilde{x}_{m}},
$$

it follows from the strict montony of the function $g(v)=\frac{\log (2 a v)}{\log (2 v)}$ for $v \in(0,1 / 2)$ that $\tilde{C}_{m}=C_{m}^{*}$ and also, due to equation (19), $\tilde{C}_{f}=C_{f}^{*}$. Finally, due to the strict monotony of the function $C(\theta)$, it follows $\tilde{\theta}_{m}=\theta_{m}^{*}$ and $\tilde{\theta}_{f}=\theta_{f}^{*}$.

Furthermore, the identifiability of the disease locus position for ASPs in a sex-specific MOD score analysis could experimentally be confirmed using the scenario of the main simulation study with $5 \mathrm{cM}$ marker-trait locus distance (Appendix Table 7). The results further indicated that the position is also identifiable using 3-G pedigrees, even in the case of no sex-specific differences in the recombination fraction.

\begin{tabular}{|c|c|c|c|c|}
\hline \multirow{3}{*}{$\begin{array}{l}\text { True marker-trait locus distance: } 5 \mathrm{cM} \\
\text { Map ratio }\end{array}$} & \multicolumn{4}{|c|}{ Estimated median marker-trait locus distance (MAD) } \\
\hline & \multicolumn{2}{|r|}{600 ASPs } & \multicolumn{2}{|c|}{$653-G$ pedigrees } \\
\hline & additive & recessive & additive & recessive \\
\hline $1: 1$ & 21 (14.83) & $9(5.93)$ & $3(4.45)$ & $5(7.41)$ \\
\hline $7: 3$ & $5(7.41)$ & $5(4.45)$ & $4(5.93)$ & $5(7.41)$ \\
\hline 9:1 & $5(7.41)$ & $5(2.97)$ & $4(5.93)$ & $5(4.45)$ \\
\hline
\end{tabular}

Table 7: Estimated median marker-trait locus distances under $H_{0, b}$ : Linkage, no imprinting when using the sex-specific map in the nonimprinting analysis as employed for the simulation.

MAD: median absolute deviation, adjusted by a constant (1.4826) for asymptotically normal consistency. ASP: affected sib-pair; 3-G pedigree: three-generation pedigree.

\section{Appendix}

\section{C.1 Description and results of the newly developed MOBIT permutation procedure (method perm)}

In brief, replicates under the null hypothesis of an expected imprinting effect of 0 , conditional on the linkage information of the real dataset, are generated by leaving the marker genotypes of the real dataset untouched, while the parental origin of the alleles in the offspring is randomly permuted for each permutation unit in a given pedigree. Here, a permutation unit is defined as a nuclear family or, in the case of half-sibs, as a set of nuclear families, whose parents are connected by matings with joint offspring. After identification of permutation units, randomization of imprinting information is achieved by manipulation of the parametric disease-locus likelihood, where each inheritance vector in the Lander-Green algorithm (Lander \& Green, 1987), on which GHM is based, is assigned a score as a function of the disease allele frequency and the penetrance values (see also Brugger and Strauch (2014) for more information on the Lander-Green algorithm and the disease-locus likelihood as implemented in GHM). Each inheritance vector score is then weighted by the corresponding probability of the marker genotype data given the particular inheritance vector. It can be shown that certain 
inheritance vectors can be collapsed into classes on the basis of the equality of their disease-locus likelihood contribution (Brugger \& Strauch, 2014). In the case of imprinting, where two heterozygote penetrances are modeled, there is a larger number of inheritance vector classes than without imprinting. This is because certain inheritance vector classes without imprinting split up in two or more classes by replacing each factor of the heterozygote penetrance $\left(f_{1}\right)$ by the corresponding parental-origin-specific value $\left(f_{1 \text { pat }}\right.$ or $\left.f_{1 \text { mat }}\right)$. It is these inheritance vector classes that get randomly permuted for a given permutation unit of a pedigree in each new replicate. It is of note, however, that confounding between imprinting and sex-specific recombination fractions cannot be avoided using this permutation procedure if the truly underlying sex-specific maps are not used in the analysis. If sex-specific maps are used in the analysis, they are correctly handled by the permutation procedure. In order to investigate the properties of the MOBIT permutation distribution, we picked a dataset from the main simulation study and calculated the corresponding permutation distribution as well as the $p$ value for this dataset. An overview of the investigated scenarios can be found in Appendix Table 8. In the case of ASPs, we also graphically depicted the resulting points in terms of allele-sharing for the permuted replicates in the tetrahedral parameter space (Appendix Figure 10 and Appendix Figure 11). This illustrates that the perm method described here leads to a sample space that is different from the one obtained for the bfnm method, which is similar to those depicted in Figure 1.

Table 8: Overview of the simulated scenarios to investigate the properties of the two proposed MOBIT simulation/permutation procedures.

\begin{tabular}{|c|c|c|c|c|c|c|}
\hline \multirow[b]{2}{*}{ Hypothesis } & \multicolumn{6}{|c|}{$\begin{array}{l}\text { Overview of the investigated scenarios for the evaluation of the MOBIT simulation/permutation } \\
\text { procedures }\end{array}$} \\
\hline & \multicolumn{2}{|c|}{$\begin{array}{l}H_{0, a}: \text { No linkage, no } \\
\text { imprinting }\end{array}$} & \multicolumn{2}{|c|}{$H_{0, b}:$ Linkage, no imprinting } & \multicolumn{2}{|c|}{$H_{1}$ : Linkage, imprinting } \\
\hline Trait model & \multicolumn{4}{|c|}{ additive/recessive } & \multicolumn{2}{|l|}{$I=0.4$} \\
\hline Map ratio & $1: 1$ & $9: 1$ & $1: 1$ & 9:1 & & 9:1 \\
\hline Analysis type & sex-averaged & sex-specific & sex-averaged & $\begin{array}{l}\text { sex- } \\
\text { averaged/sex- } \\
\text { specific }\end{array}$ & sex-averaged & sex-specific \\
\hline Pedigree type & Sample size & $\begin{array}{l}\text { Number of } \\
\text { replicates }\end{array}$ & & & & \\
\hline ASP & 600 & 10,000 & & & & \\
\hline 3-G pedigree & 65 & 10,000 & & & & \\
\hline \multicolumn{7}{|c|}{ Scenario: 1 marker, $5 \mathrm{cM}$ (sex-averaged) between markerand disease locus } \\
\hline \multirow{2}{*}{\multicolumn{7}{|c|}{$\begin{array}{l}\text { Segregation of additive trait simulated with penetrances }\left\{f_{0}, f_{1}, f_{2}\right\}=\{0.03,0.13,0.23\} \text { and disease allele frequency } p= \\
0.1 \\
\text { Segregation of recessive trait simulated with penetrances }\left\{f_{0}, f_{1}, f_{2}\right\}=\{0.05,0.05,0.90\} \text { and disease allele frequency } p \\
=0.2\end{array}$}} \\
\hline & & & & & & \\
\hline \multicolumn{7}{|c|}{ Maternal imprinting simulations for additive trait model with $I=\frac{f_{1 \text { pat }}-f_{1 \text { mat }}}{f_{2}-f_{0}}=0.4$} \\
\hline
\end{tabular}

ASP: affected sib-pair; 3-G pedigree: three-generation pedigree.

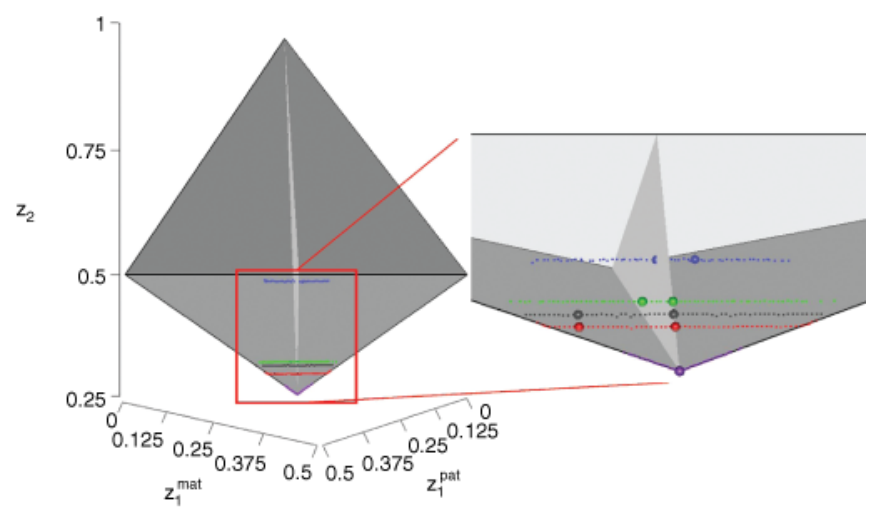


Figure 10: Results of the MOBIT permutation procedure (method perm) using a sex-averaged map for the analysis. The illustration is based on the tetrahedral parameter space $\mathbf{T}$ for affected sib-pairs (ASPs, see also Figure 1). Left subfigure: total view of $\mathbf{T}$; right subfigure: detailed view (slightly rotated to the right) on the points in terms of allele-sharing. For more details as to the investigated hypotheses and trait models see Appendix Table 8 and Appendix Table 9. Bullets correspond to points in terms of allele-sharing according to the trait-model parameters and the recombinaion fraction obtained from the MOD (bullets on the possible triangle) and IMOD score analyses of the picked replicate, which was used as the real dataset. In some cases, the position of the MOD and IMOD score bullets cannot be visibly distinguished. Points correspond to allele-sharing IMOD score estimates obtained for each of the 10,000 generated replicates of the MOBIT permutation procedure. The color scheme of bullets and dots is as follows. Purple: no linkage, no imprinting $\left(H_{0, a}\right)$ with 1:1 map ratio; green: linkage, no imprinting $\left(H_{0, b}\right.$, additive trait model) with $5 \mathrm{cM}$ marker-trait locus distance and 1:1 map ratio; blue: $H_{0, b}$ (recessive trait model) with $5 \mathrm{cM}$ marker-trait locus distance and 1:1 map ratio; red: $H_{0, b}$ (additive trait model) with $5 \mathrm{cM}$ marker-trait locus distance and 9:1 map ratio; grey: linkage and imprinting $\left(H_{1}\right)$ with $5 \mathrm{cM}$ markertrait locus distance and 1:1 map ratio.

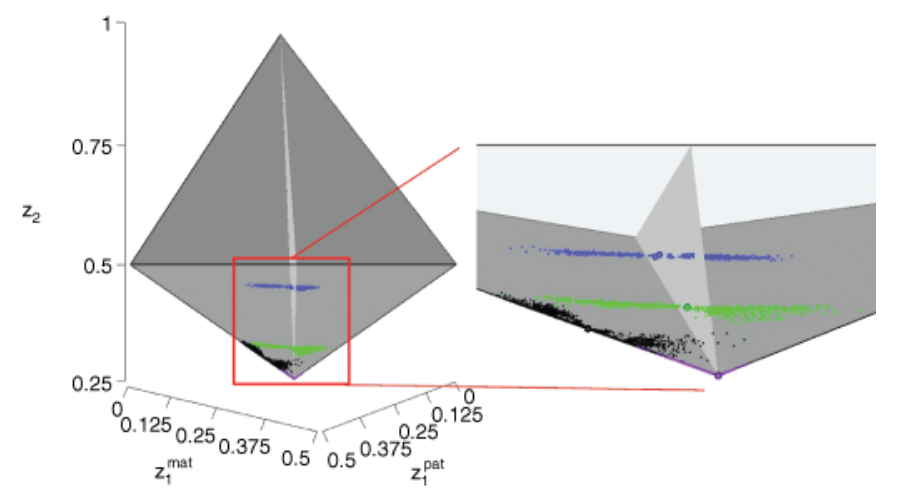

Figure 11: Results of the MOBIT permutation procedure (method perm) using the correct sex-specific map with a 9:1 female/male map ratio for the analysis. The illustration is based on the tetrahedral parameter space $\mathbf{T}$ for affected sib-pairs (ASPs, see also Figure 1). Left subfigure: total view of T; right subfigure: detailed view (slightly rotated to the right) on the points in terms of allele-sharing. Bullets correspond to points in terms of allele-sharing according to the trait-model parameters and the recombinaion fraction obtained from the MOD and IMOD score analyses of the picked replicate, which was used as the real dataset. The left of the two blue bullet points corresponds to the best-fitting nonimprinting model obtained from the MOD score analysis. In all other cases, the position of the MOD and IMOD score bullets cannot be visibly distinguished. Points correspond to allele-sharing IMOD score estimates obtained for each of the 10,000 generated replicates of the MOBIT permutation procedure. The color scheme of bullets and dots is as follows. Purple: no linkage, no imprinting $\left(H_{0, a}\right)$ with 9:1 map ratio; green: linkage, no imprinting $\left(H_{0, b}\right.$, additive trait model) with $5 \mathrm{cM}$ marker-trait locus distance and 9:1 map ratio; blue: $H_{0, b}$ (recessive trait model) with $5 \mathrm{cM}$ marker-trait locus distance and 9:1 map ratio; grey: linkage and imprinting $\left(H_{1}\right)$ with $5 \mathrm{cM}$ marker-trait locus distance and 9:1 map ratio. 


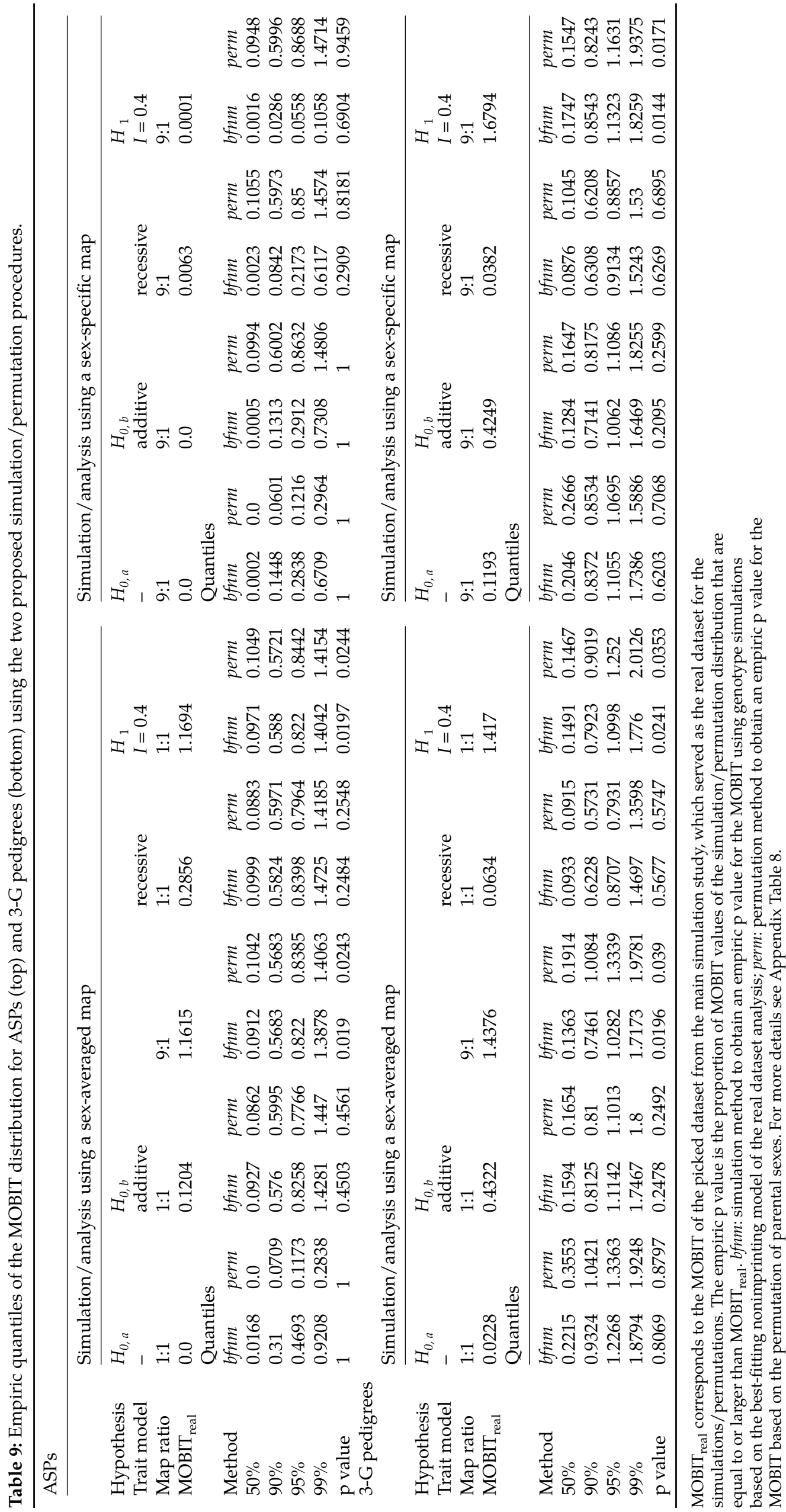




\section{C.1.1 Permutation distribution for ASPs}

As can be seen from the results of the sex-averaged ASP analyses in Appendix Figure 10, the permutation procedure generated replicates with an expected equal allele-sharing between the parental sexes on the possible triangle. Points in terms of randomly unequal parental allele-sharing, which correspond to the permutation replicates, were stretched out on a more or less straight line perpendicular to the possible triangle, except for the point of no linkage, where these points were lined up on the outer edges of the tetrahedron. It is of note that the best-fitting nonimprinting model for every replicate always corresponded to the same point in terms of allele-sharing as for the real dataset, which illustrates that the permutation procedure in fact generates replicates under the conditional null hypothesis mentioned above. The same held also true for the permutations using the sex-specific map in Appendix Figure 11, however, the expected point in terms of allele-sharing was shifted into the left half-tetrahedron according to the underlying map ratio. In the case of the point of no linkage, replicates were lined up on either edge of the tetrahedron according to the map ratio. As can be deduced from the points in terms of allele-sharing of replicates for the dataset originally simulated under $H_{1}$ (black points, Appendix Figure 11), the permutation procedure reflects peculiarities and boundaries of the parameter space as well as effects due to sex-specific recombination fractions. With regard to the scenario in which a truly underlying 9:1 map ratio is not taken into account in the analysis (red points, Appendix Figure 10), the corresponding quantiles were comparable to those of the 1:1 scenarios, indicating that confounding cannot be prevented using such a permutation procedure. The $95 \%$ quantiles of the $H_{0, b}$ and $H_{1}$, scenarios using a sex-averaged map for method perm (see Appendix Table 9, top) were all similar to each other. Furthermore, the corresponding quantiles of the sex-specific analyses were quite similar to the sex-averaged ones, which shows that the MOBIT permutation distribution is less affected by maximization curves, according to the sex-specific map ratio, than the $b f n m$ method (see Appendix Table 9, top). The latter effect, however, may cause substantial differences in quantiles and $p$ values between the two methods, and hence also regarding the conclusion whether imprinting is present or not.

\section{C.1.2 Permutation distribution of $3-\mathrm{C}$ pedigrees}

The results of the MOBIT permutation distribution for 3-G pedigrees can be found in Appendix Table 9, bottom. The properties of the quantiles were comparable to those for ASPs and seemed to reflect peculiarities of the 3-G pedigree parameter space as can be deduced from the different quantiles for additive and recessive trait models. Similar to ASPs, quantiles for the method perm using a sex-specific map in the analysis were also comparable to those obtained from the corresponding analyses using a sex-averaged map. Unlike for ASPs, the quantiles for the perm method differed from those obtained by the bfnm method not only when using a sex-specific map, but also when using a sex-averaged map in the analysis.

\section{References}

Abecasis, G. R., S. S. Cherny, W. O. Cookson and L. R. Cardon (2002): “Merlin-rapid analysis of dense genetic maps using sparse gene flow trees," Nat. Genet., 30, 97-101.

Bain, S. C., B. R. Rowe, A. H. Barnett and J. A. Todd (1994): "Parental origin of diabetes-associated HLA types in sibling pairs with type I diabetes," Diabetes, 43, 1462-1468.

Brugger, M., S. Rospleszcz and K. Strauch (2016): “Estimation of trait model parameters in a MOD score linkage analysis,” Hum. Hered., 82, 103-139.

Brugger, M. and K. Strauch (2014): “Fast linkage analysis with MOD scores using algebraic calculation," Hum. Hered., 78, $179-194$.

Christensen, U., S. Møller-Larsen, M. Nyegaard, A. Haagerup, A. Hedemand, C. Brasch-Andersen, T. A. Kruse, T. J. Corydon, M. Deleuran and A. D. Børglum (2009): “Linkage of atopic dermatitis to chromosomes 4q22, 3p24 and 3q21," Hum. Cenet., 126, 549-557.

Churchill, C. A. and R. W. Doerges (2008): "Naive application of permutation testing leads to inflated type l error rates," Cenetics, 178, 609610.

Clerget-Darpoux, F., C. Bonaïti-Pellié and ]. Hochez (1986): “Effects of misspecifying genetic parameters in lod score analysis,” Biometrics, 42, 393-399.

Davies, W., A. R. Isles and L. S. Wilkinson (2005): “Imprinted gene expression in the brain,” Neurosci. Biobehav. Rev., 29, 421-430.

Daw, E. W., E. A. Thompson and E. M. Wijsman (2000): “Bias in multipoint linkage analysis arising from map misspecification," Cenet. Epidemiol., 19, 366-380.

Dib, C., S. Fauré, C. Fizames, D. Samson, N. Drouot, A. Vignal, P. Millasseau, S. Marc, J. Hazan, E. Seboun, M. Lathrop, G. Gyapay, J. Morissette and J. Weissenbach (1996): “A comprehensive genetic map of the human genome based on 5,264 microsatellites," Nature, 380, $152-154$.

Dietter, J., M. Mattheisen, R. Fürst, F. Rüschendorf, T. F. Wienker and K. Strauch (2007): “Linkage analysis using sex-specific recombination fractions with GENEHUNTER-MODSCORE," Bioinformatics, 23, 64-70. 
Dong, C., W.-D. Li, F. Geller, L. Lei, D. Li, O. Y. Corlova, J. Hebebrand, C. I. Amos, R. D. Nicholls and R. A. Price (2005): “Possible genomic imprinting of three human obesity-related genetic loci," Am. J. Hum. Cenet., 76, 427-437.

Elston, R. C. (1989): "Man bites dog? The validity of maximizing lod scores to determine mode of inheritance," Am. J. Med. Cenet., 34, 487488.

Falls, J. G., D. J. Pulford, A. A. Wylie and R. L. Jirtle (1999): “Cenomic imprinting: implications for human disease,” Am. J. Pathol., 154, 635-647.

Feng, R. and H. Zhang (2008): “A genomic imprinting test for ordinal traits in pedigree data," Cenet. Epidemiol., 32, 132-142.

Fingerlin, T. E., G. R. Abecasis and M. Boehnke (2006): "Using sex-averaged genetic maps in multipoint linkage analysis when identity-bydescent status is incompletely known," Cenet. Epidemiol., 30, 384-396.

Fishelson, M., and D. Ceiger (2002): “Exact genetic linkage computations for general pedigrees,” Bioinformatics, 18(Suppl 1), S189-S198.

Flaquer, A., C. Baumbach, E. Piñero, F. Carcía Algas, M. A. de la Fuente Sanchez, J. Rosell, J. Toquero, L. Alonso-Pulpon, P. Garcia-Pavia, K. Strauch and D. Heine-Suñer (2013): “Cenome-wide linkage analysis of congenital heart defects using MOD score analysis identifies two novel loci," BMC Cenet., 14, 44.

Flaquer, A., C. Fischer and T. F. Wienker (2009): "A new sex-specific genetic map of the human pseudoautosomal regions (PAR1 and PAR2)," Hum. Hered., 68, 192-200.

Flaquer, A. and K. Strauch (2012): "A comparison of different linkage statistics in small to moderate sized pedigrees with complex diseases," BMC Res. Notes, 5, 411.

Greenberg, D. A., M. Durner, M. Keddache, S. Shinnar, S. R. Resor, S. L. Moshe, D. Rosenbaum, J. Cohen, C. Harden, H. Kang, S. Wallace, D. Luciano, K. Ballaban-Cil, L. Tomasini, G. Zhou, I. Klotz and E. Dicker (2000): “Reproducibility and complications in gene searches: linkage on chromosome 6, heterogeneity, association, and maternal inheritance in juvenile myoclonic epilepsy," Am. J. Hum. Cenet., 66, 508516.

Greenberg, D. A., M. C. Monti, B. Feenstra, J. Zhang and S. E. Hodge (2010): “The essence of linkage-based imprinting detection: comparing power, type 1 error, and the effects of confounders in two different analysis approaches," Ann. Hum. Cenet., 74, 248-262.

Gudbjartsson, D. F., K. Jonasson, M. L. Frigge and A. Kong (2000): “Allegro, a new computer program for multipoint linkage analysis," Nat. Cenet., 25, 12-13.

Gudbjartsson, D. F., T. Thorvaldsson, A. Kong, G. Cunnarsson and A. Ingolfsdottir (2005): “Allegro version 2,” Nat. Genet., 37, $1015-1016$.

Hager, R., J. M. Cheverud and J. B. Wolf (2008): "Maternal effects as the cause of parent-of-origin effects that mimic genomic imprinting," Genetics, 178, 1755-1762.

Han, M., Y.-Q. Hu and S. Lin (2013): "Joint detection of association, imprinting and maternal effects using all children and their parents," Eur. J. Hum. Genet., 21, 1449-1456.

Hanson, R. L., S. Kobes, R. S. Lindsay and W. C. Knowler (2001): “Assessment of parent-of-origin effects in linkage analysis of quantitative traits," Am. J. Hum. Cenet., 68, 951-962.

Hoggart, C. J., G. Venturini, M. Mangino, F. Comez, C. Ascari, J. H. Zhao, A. Teumer, T. W. Winkler, N. Tšernikova, J. Luan, E. Mihailov, G. B. Ehret, W. Zhang, D. Lamparter, T. O. Esko, A. Macé, S. Rüeger, P.-Y. Bochud, M. Barcella, Y. Dauvilliers, B. Benyamin, D. M. Evans, C. Hayward, M. F. Lopez, L. Franke, A. Russo, I. M. Heid, E. Salvi, S. Vendantam, D. E. Arking, E. Boerwinkle, J. C. Chambers, C. Fiorito, H. Grallert, S. Guarrera, G. Homuth, J. E. Huffman, D. Porteous, D. Moradpour, A. Iranzo, J. Hebebrand, J. P. Kemp, G. J. Lammers, V. Aubert, M. H. Heim, N. G. Martin, G. W. Montgomery, R. Peraita-Adrados, J. Santamaria, F. Negro, C. O. Schmidt, R. A. Scott, T. D. Spector, K. Strauch, H. Völzke, N. J. Wareham, W. Yuan, J. T. Bell, A. Chakravarti, J. S. Kooner, A. Peters, C. Matullo, H. Wallaschofski, J. B. Whitfield, F. Paccaud, P. Vollenweider, S. Bergmann, J. S. Beckmann, M. Tafti, N. D. Hastie, D. Cusi, M. Bochud, T. M. Frayling, A. Metspalu, M.-R. Jarvelin, A. Scherag, C. D. Smith, I. B. Borecki, V. Rousson, J. N. Hirschhorn, C. Rivolta, R. J. F. Loos and Z. Kutalik (2014): “Novel approach identifies SNPs in SLC2a10 and KCNK9 with evidence for parent-of-origin effect on body mass index," PLoS Cenet., 10, e1004508.

Holmans, P. (1993): “Asymptotic properties of affected-sib-pair linkage analysis,” Am. J. Hum. Cenet., 52, 362-374.

Knapp, M. (2005): “A note on linkage analysis with affected sib triplets," Hum. Hered., 59, 21-25.

Knapp, M., S. A. Seuchter and M. P. Baur (1994): “Linkage analysis in nuclear families. 2: Relationship between affected sib-pair tests and lod score analysis," Hum. Hered., 44, 44-51.

Knapp, M. and K. Strauch (2004): “Affected-sib-pair test for linkage based on constraints for identical-by-descent distributions corresponding to disease models with imprinting," Cenet. Epidemiol., 26, 273-285.

Kong, A. and N. J. Cox (1997): "Allele-sharing models: LOD scores and accurate linkage tests," Am. J. Hum. Cenet., 61, 1179-1188.

Kruglyak, L., M. J. Daly, M. P. Reeve-Daly and E. S. Lander (1996): “Parametric and nonparametric linkage analysis: a unified multipoint approach," Am. J. Hum. Cenet., 58, 1347-1363.

Kruse, L. V., M. Nyegaard, U. Christensen, S. Møller-Larsen, A. Haagerup, M. Deleuran, L. G. Hansen, S. K. Ven $\varnothing$, D. Coossens, J. Del-Favero and A. D. Børglum (2012): “A genome-wide search for linkage to allergic rhinitis in Danish sib-pair families," Eur. J. Hum. Cenet., 20, 965972.

Kurz, T., J. Altmueller, K. Strauch, F. Rüschendorf, A. Heinzmann, M. F. Moffatt, W.O.C. M. Cookson, F. Inacio, P. Nürnberg, H. H. Stassen and K. A. Deichmann (2005): "A genome-wide screen on the genetics of atopy in a multiethnic European population reveals a major atopy locus on chromosome 3q21.3," Allergy, 60, 192-199.

Kurz, T., K. Strauch, A. Heinzmann, S. Braun, M. Jung, F. Rüschendorf, M. F. Moffatt, W. O. Cookson, F. Inacio, A. Ruffilli, G. Nordskov-Hansen, G. Peltre, J. Forster, J. Kuehr, A. Reis, T. F. Wienker and K. A. Deichmann (2000): “A European study on the genetics of mite sensitization," J. Allergy Clin. Immunol., 106, 925-932.

Lander, E. S. and P. Green (1987): Construction of multilocus genetic linkage maps in humans," Proc. Natl. Acad. Sci. U.S.A., 84, $2363-2367$.

Lemire, M. (2005): "A simple nonparametric multipoint procedure to test for linkage through mothers or fathers as well as imprinting effects in the presence of linkage," BMC Cenet., 6(Suppl 1), S159.

Lemire, M. (2006): “SUP: an extension to SLINK to allow a larger number of marker loci to be simulated in pedigrees conditional on trait values," BMC Cenet., 7, 40.

Lewis, A. and W. Reik (2006): “How imprinting centres work," Cytogenet. Cenome Res., 113, 81-89. 
Liu, X.-Q., C. Greenwood, K.-S. Wang and A. Paterson (2005): “A genome scan for parent-of-origin linkage effects in alcoholism,” BMC Genet., 6(Suppl 1), S160.

Maeda, N. and Y. Hayashizaki (2006): “Cenome-wide survey of imprinted genes,” Cytogenet. Cenome Res., 113, 144-152.

Matise, T. C., F. Chen, W. Chen, F. M. D. L. Vega, M. Hansen, C. He, F. C. L. Hyland, G. C. Kennedy, X. Kong, S. S. Murray, J. S. Ziegle, W. C. L. Stewart and S. Buyske (2007): "A second-generation combined linkage physical map of the human genome," Cenome Res., 17, 17831786.

Mattheisen, M., J. Dietter, M. Knapp, M. P. Baur and K. Strauch (2008): “Inferential testing for linkage with CENEHUNTER-MODSCORE: the impact of the pedigree structure on the null distribution of multipoint MOD scores," Cenet. Epidemiol., 32, 73-83.

Metz, C. W. (1938): “Chromosome behaviour, inheritance and sex determination in Sciara," Am. Nat., 72, 485-520.

Moffatt, M. F. and W. O. Cookson (1998): “The genetics of asthma. Maternal effects in atopic disease," Clin. Exp. Allergy, 28(Suppl 1), 56-61; discussion 65-6.

Mukhopadhyay, N. and D. E. Weeks (2003): "Linkage analysis of adult height with parent-of-origin effects in the Framingham Heart Study," BMC Cenet., 4(Suppl 1), S76.

Ott, J. (1989): “Computer-simulation methods in human linkage analysis," Proc. Natl. Acad. Sci. U.S.A., 86, 4175-4178.

Pàldi, A., G. Gyapay and J. Jami (1995): "Imprinted chromosomal regions of the human genome display sex-specific meiotic recombination frequencies," Curr. Biol., 5, 1030-1035..

Paterson, A. D., D. M. Naimark and A. Petronis (1999): The analysis of parental origin of alleles may detect susceptibility loci for complex disorders," Hum. Hered., 49, 197-204.

Risch, N. (1984): “Segregation analysis incorporating linkage markers. I. Single-locus models with an application to type I diabetes," Am. J. Hum. Genet., 36, 363-386.

Schäffer, A. A., M. Lemire, J. Ott, C. M. Lathrop and D. E. Weeks (2011): “Coordinated conditional simulation with SLINK and SUP of many markers linked or associated to a trait in large pedigrees," Hum. Hered., 71, 126-134.

Schumacher, J., R. Kaneva, R. A. Jamra, G. O. Diaz, S. OhIraun, V. Milanova, Y.-A. Lee, F. Rivas, F. Mayoral, R. Fuerst, A. Flaquer, C. Windemuth, E. Gay, S. Sanz, M. J. González, S. Gil, F. Cabaleiro, F. del Rio, F. Perez, J. Haro, C. Kostov, V. Chorbov, A. Nikolova-Hill, V. Stoyanova, G. Onchev, I. Kremensky, K. Strauch, T. G. Schulze, P. Nürnberg, W. Gaebel, A. Klimke, G. Auburger, T. F. Wienker, L. Kalaydjieva, P. Propping, S. Cichon, A. Jablensky, M. Rietschel and M. M. Nöthen (2005): “Cenomewide scan and fine-mapping linkage studies in four European samples with bipolar affective disorder suggest a new susceptibility locus on chromosome 1p35-p36 and provides further evidence of loci on chromosome 4q31 and 6q24," Am. J. Hum. Cenet., 77, 1102-1111.

Self, S. and K. Liang (1987): “Asymptotic properties of maximum likelihood estimators and likelihood ratio tests under nonstandard conditions," J. Am. Stat. Assoc., 82, 605-610.

Shete, S. and X. Zhou (2005): “Parametric approach to genomic imprinting analysis with applications to Angelman's syndrome," Hum. Hered., 59, 26-33.

Shete, S., X. Zhou and C. I. Amos (2003): “Cenomic imprinting and linkage test for quantitative-trait loci in extended pedigrees," Am. J. Hum. Cenet., 73, 933-938.

Shute, N. C. and W. J. Ewens (1988): “A resolution of the ascertainment sampling problem. III. Pedigrees,” Am. J. Hum. Cenet., 43, 387-395

Sieberts, S. K. and D. F. Gudbjartsson (2005): "Sex-specific maps and consequences for linkage mapping," In Lynn Jorde, Peter Little, Mike Dunn, and Shankar Subramaniam, (Eds.), Encyclopedia of Cenetics, Cenomics, Proteomics and Bioinformatics. John Wiley \& Sons, Chichester, UK.

Smalley, S. L. (1993): “Sex-specific recombination frequencies: a consequence of imprinting?,” Am. J. Hum. Genet., 52, $210-212$.

Solter, D. (2006): “Imprinting today: end of the beginning or beginning of the end?" Cytogenet. Cenome Res., 113, $12-16$.

Spencer, H. C. (2009): “Effects of genomic imprinting on quantitative traits," Cenetica, 136, 285-293.

Stine, O. C., J. Xu, R. Koskela, F. J. McMahon, M. Gschwend, C. Friddle, C. D. Clark, M. G. Mclnnis, S. G. Simpson, T. S. Breschel, E. Vishio, K. Riskin, H. Feilotter, E. Chen, S. Shen, S. Folstein, D. A. Meyers, D. Botstein, T. G. Marr and J. R. DePaulo (1995): “Evidence for linkage of bipolar disorder to chromosome 18 with a parent-of-origin effect," Am. J. Hum. Cenet., 57, 1384-1394.

Strauch, K. (2003): "Parametric linkage analysis with automatic optimization of the disease model parameters," Am. J. Hum. Cenet., 73, A2624.

Strauch, K. (2005): “Cene mapping, imprinting and epigenetics,” In Lynn Jorde, Peter Little, Mike Dunn, and Shankar Subramaniam, (Eds.), Encyclopedia of Cenetics, Cenomics, Proteomics and Bioinformatics. John Wiley \& Sons, Chichester, UK.

Strauch, K. (2007): "MOD-score analysis with simple pedigrees: an overview of likelihood-based linkage methods," Hum. Hered., 64, 192202.

Strauch, K., R. Fimmers, T. Kurz, K. A. Deichmann, T. F. Wienker and M. P. Baur (2000a): “Parametric and nonparametric multipoint linkage analysis with imprinting and two-locus-trait models: application to mite sensitization," Am. J. Hum. Genet., 66, $1945-1957$.

Strauch, K., R. Fimmers, T. F. Wienker, M. P. Baur, S. Cichon, P. Propping and M. M. Nöthen (2000b): "Strauch et al reply," Mol. Psychiatry, 5 , 126-127.

Suarez, B. K., J. Rice and T. Reich (1978): “The generalized sib pair IBD distribution: its use in the detection of linkage," Ann. Hum. Cenet., 42, 87-94.

Vincent, Q., A. Alcaïs, A. Alter, E. Schurr and L. Abel (2006): “Quantifying genomic imprinting in the presence of linkage,” Biometrics, 62, 1071-1080.

Walter, J. and M. Paulsen (2003): “Imprinting and disease," Semin. Cell Dev. Biol., 14, 101-110.

Weeks, D. E. (2010): “powerpkg: Power analyses for the affected sib pair and the TDT design," R package version 1.3. https://CRAN.Rproject.org/package=powerpkg.

Weeks, D. E., T. Lehner, E. Squires-Wheeler, C. Kaufmann and ]. Ott (1990): “Measuring the inflation of the lod score due to its maximization over model parameter values in human linkage analysis," Cenet. Epidemiol., 7, 237-243.

Whittaker, J. C., N. Charani, P. Hindmarsh and M. I. McCarthy (2003): “Estimation and testing of parent-of-origin effects for quantitative traits," Am. J. Hum. Genet., 72, 1035-1039. 
Wilkins-Haug, L. (2009): “Epigenetics and assisted reproduction,” Curr. Opin. Obstet. Cynecol., 21, 201-206. Wilks, S. S. (1938): "The large-sample distribution of the likelihood ratio for testing composite hypotheses," Ann. Math. Stat., 9, 60-62.

Wu, C.-C., S. Shete and C. I. Amos (2005): "Linkage analysis of affected sib pairs allowing for parent-of-origin effects," Ann. Hum. Cenet., 69, $113-126$. 